\section{A) Check for updates}

Cite this: Chem. Soc. Rev., 2020, 49,5850

Received 2nd April 2020

DOI: $10.1039 / \mathrm{d} 0 \operatorname{cs} 00318 \mathrm{~b}$

rsc.li/chem-soc-rev

\title{
Nanomechanical mapping of soft materials with the atomic force microscope: methods, theory and applications
}

\author{
Ricardo Garcia (D)
}

\begin{abstract}
Fast, high-resolution, non-destructive and quantitative characterization methods are needed to develop materials with tailored properties at the nanoscale or to understand the relationship between mechanical properties and cell physiology. This review introduces the state-of-the-art force microscope-based methods to map at high-spatial resolution the elastic and viscoelastic properties of soft materials. The experimental methods are explained in terms of the theories that enable the transformation of observables into material properties. Several applications in materials science, molecular biology and mechanobiology illustrate the scope, impact and potential of nanomechanical mapping methods.
\end{abstract}

\section{Introduction}

The atomic force microscope (AFM) ${ }^{1}$ has greatly contributed to our current understanding of soft materials and their physical, chemical and biomolecular interactions. ${ }^{2-11}$ Mapping at highspatial resolution the mechanical properties of heterogeneous interfaces is one of the key achievements of AFM. In this review

Instituto de Ciencia de Materiales de Madrid, CSIC, c/Sor Juana Inés de la Cruz 3, 28049 Madrid, Spain. E-mail: r.garcia@csic.es

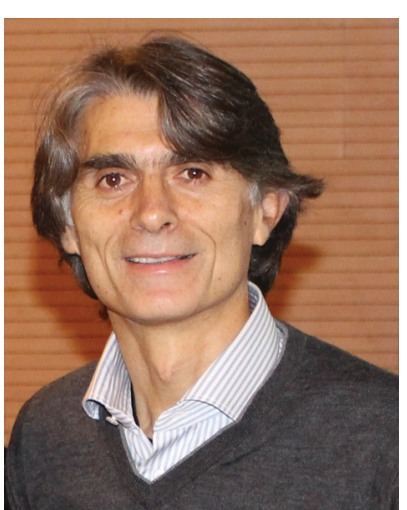

Ricardo Garcia
Ricardo Garcia is a Professor of nanoscience and nanotechnology at the Instituto de Ciencia de Materials de Madrid (CSIC). His scientific activity is focused on the development of force microscopy methods for studying soft materials and solid-liquid interfaces. In the late 90s, he contributed to the development of tapping mode AFM. More recently, his research is focused on three problems: development of multifrequency methods, in particular, bimodal AFM for high-resolution mapping of soft matter; designing 3D-AFMs for providing atomic-resolution images of solid-liquid interfaces and advancing AFM for mechanical property mapping of living cells. Some of his inventions were commercialized. nanomechanical mapping and nanomechanical force spectroscopy have the same meaning. Those terms are used interchangeably to define the field of AFM-based methods that generate maps of mechanical properties with nanoscale spatial resolution.

Several factors drive the evolution of this field. In materials science, the engineering of heterogeneous materials demands techniques to map with high-spatial resolution and quantitative accuracy the mechanical properties of the resulting interfaces. ${ }^{7}$ In mechanobiology, ${ }^{12}$ time-resolved maps of mechanical interactions are necessary to fully understand cell differentiation and growth. Finally, the capabilities of AFM to generate atomic-scale resolution images of a variety of solid-liquid interfaces ${ }^{13}$ demand fast and accurate methods to map tip-surface forces.

Nanomechanical force spectroscopy involves a variety of scientific backgrounds from applied physics to physical chemistry; from molecular biology to mechanical engineering. It involves also a diversity of goals ranging from those of academia to those of industry. Those factors have produced a wealth of experimental methods and applications. At the same time, those factors have contributed to establish a loosely connected field.

The AFM is a mechanical microscope that transforms the interaction force acting between a sharp tip and a sample surface into the displacement of a cantilever-tip transducer (Fig. 1). The capability of the AFM to generate mechanical properties maps of soft materials was recognized more than 25 years ago by Hansma and co-workers ${ }^{14,15}$ In fact, one of the early achievements of AFM was the nanoscale characterization of polymer interfaces in their native state. ${ }^{16,17}$ Since then, a large variety of methods have been proposed, improved and 

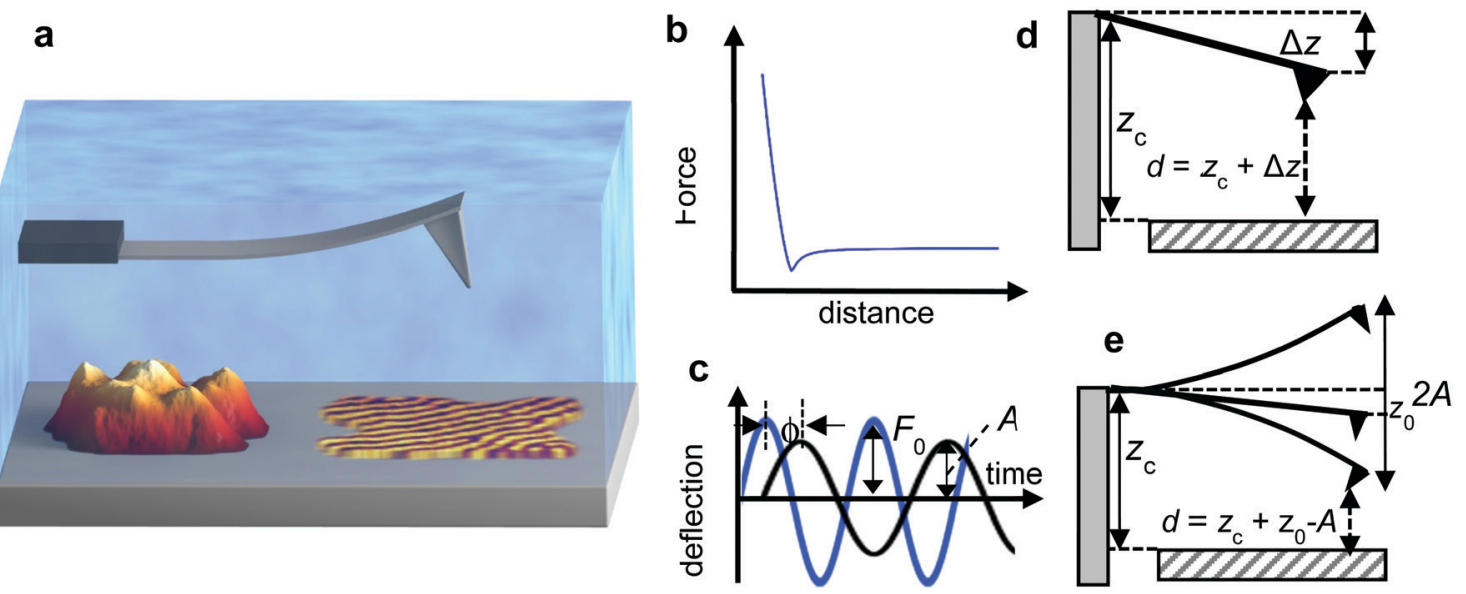

Fig. 1 AFM, force-distance curves and observables. (a) Scheme of an heterogenous interface made of regions of different composition and morphology. (b) Force-distance curve. (c) Driving force (blue) and tip's oscillation in the presence of a force (black). Definition of phase shift. (d) Scheme of the distances and deflections in an off-resonance AFM measurement. (e) Scheme of the distances and deflections in an on-resonance AFM measurement.

applied to characterize soft materials. ${ }^{18-26}$ Some of the earlier methods such as force-volume ${ }^{4,14,18,19}$ and AFM phase-imaging ${ }^{16,17}$ are still evolving while the applications of other methods have declined. $^{26}$

The main goal of this review is to introduce the experimental approaches, the theoretical background and the capabilities of the most advanced AFM-based methods to map at very high $(<10 \mathrm{~nm})$ or high-spatial resolution $(10-100 \mathrm{~nm})$ the mechanical properties of soft materials. Soft materials are loosely defined as materials with an elastic modulus within the $100 \mathrm{~Pa}$ to $10 \mathrm{GPa}$ range. Cells, proteins, polymers, biopolymers or liposomes are some examples of soft materials.

An advanced nanomechanical mapping method should have the following features: (i) high-spatial resolution; (ii) quantitative accuracy; (iii) independent of the tip's properties; (iv) data acquisition in the high-speed range ( $\geq 1$ frame per $s)$ and (v) suppression of cross-talk between topography and material properties.

The review is divided in four sections. The first section introduces the scientific context. It provides a classification of nanomechanical force spectroscopy methods in two main categories, force-distance curve and parametric methods. The second section presents the theoretical concepts needed to understand the relationship between the AFM observables and the mechanical properties of a material. Elastic, inelastic and viscoelastic properties are considered. The relevance of finite element method and molecular dynamics simulations to interpret nanomechanical maps are also discussed. A special sub-section is devoted to introduce the challenges posed by the characterization of the mechanical properties of living cells.

The third section describes the most widely used nanomechanical mapping methods, among them force-volume, bimodal AFM, AFM phase-imaging and contact resonance. This section is illustrated by several applications in materials science, surface science or cell biology. The last section provides an analysis of some fundamental and open issues. It examines some of the technological challenges that are likely to shape the evolution of the field.

\section{Classification of nanomechanical mapping methods}

The AFM measures the mechanical properties of a surface either by recording the interaction force with respect to the tip-surface distance (Fig. 1b) or by measuring the effect of the interaction force on some parameters of the tip's oscillation (Fig. 1c).

A plethora of AFM-based methods has been proposed to generate spatially-resolved maps of mechanical properties. Initially, the methods were classified according to the AFM mode used to generate a topographic image, quasi-static (off-resonance) or dynamic (on-resonance). This classification scheme is useful but incomplete because it does not inform on how mechanical properties are obtained.

A summary of the nanomechanical mapping methods is presented in Fig. 2a. The methods might be classified according to different features such as the quantitative character, the type of observable or the ratio between the resonant frequency and the modulation frequency. The most distinctive classification comes from how values of mechanical properties are obtained. Forcedistance curves methods record a force-distance curve on each point of the surface (Fig. 2b). In parametric methods, some observables such as the amplitude, the phase or the frequency shifts are recorded on each point of the surface (Fig. 2c). An analytical theory transforms those observables in terms of parameters of the interaction force.

\subsection{Force-distance curve methods}

A force-distance curve (FDC) provides the dependence of the force with respect to the tip-surface distance on each point of the surface. Force-distance curves are determined either by 




Fig. 2 Classification of nanomechanical mapping methods. (a) The scheme is based on how mechanical properties are obtained. (b) Scheme of a forcedistance curve method. Off-resonance methods lie to the left of the dashed line. On-resonance methods lie to the right of the dashed line. (c) Scheme of nanomechanical mapping by using a parametric method. $A, \phi$ and $f$ are, respectively, the amplitude, phase shift and resonant frequency of the tip. Those parameters depend on the sample properties.

driving the cantilever at a frequency well below its fundamental resonance (off-resonance) or at a frequency that coincides or is very close to its fundamental resonance (on-resonance). Fig. 3 shows some theoretical force-distance curves. The force might also be represented with respect to time by knowing the waveform of the tip-surface distance.

In an off-resonance method the deflection is recorded while the tip-surface distance is modulated at a frequency much smaller than the fundamental resonance. The postulate of force spectroscopy ${ }^{27}$ is applied to transform deflections into forces. The resulting FDC is fitted to a contact mechanics model (see below). Force-volume (FV) ${ }^{4,14,15,18,19}$ is by far the most common AFM-based force-distance curve method. Force-volume is implemented in most commercial AFMs under different names. ${ }^{28}$ In the early days, the modulation frequencies were of a few $\mathrm{Hz}$ which led to identification of quasi-static and off-resonance methods.

On-resonance methods can also be used to generate forcedistance curves. ${ }^{29-31}$ In this case, the cantilever is excited at its fundamental resonance. A FDC might be determined by measuring and processing the Fourier components of the force while imaging ${ }^{29,30}$ or by recording amplitude and phase-shift distance curves. ${ }^{31}$

\subsection{Parametric methods}

In a parametric method an analytic model relates the observables to some mechanical properties. Parametric methods are associated with the excitation and/or detection of the cantilever deflection at one or several of its resonances and/or a multiple of the fundamental resonance (higher harmonic). Resonances are properties of the mechanical system while an harmonic is an integer multiple of the drive frequency. ${ }^{20}$ Bimodal $\mathrm{AFM}^{20,32}$ or contact resonance $\mathrm{AFM}^{33}$ are examples of parametric methods.

\section{Theory of nanomechanical force spectroscopy. Equation of motion}

The aim is to introduce the theory or theories that enable the transformation of observables into mechanical properties of a sample. A theory for AFM-based mechanical measurements involves three main steps, the equation of motion, the model for the interaction force and the solution of the equation of motion for a given model. Fig. 4 schematizes some tip-sample interfaces.

\subsection{Equation of motion}

A nanomechanical force spectroscopy measurement involves the relative displacement of the tip with respect to the sample surface. The motion of a cantilever-tip system is based on the Euler-Bernoulli equation for a continuous and threedimensional beam..$^{31,34-36}$ If the tip is attached at the free end of the cantilever and the interaction force acts on the tip, the 

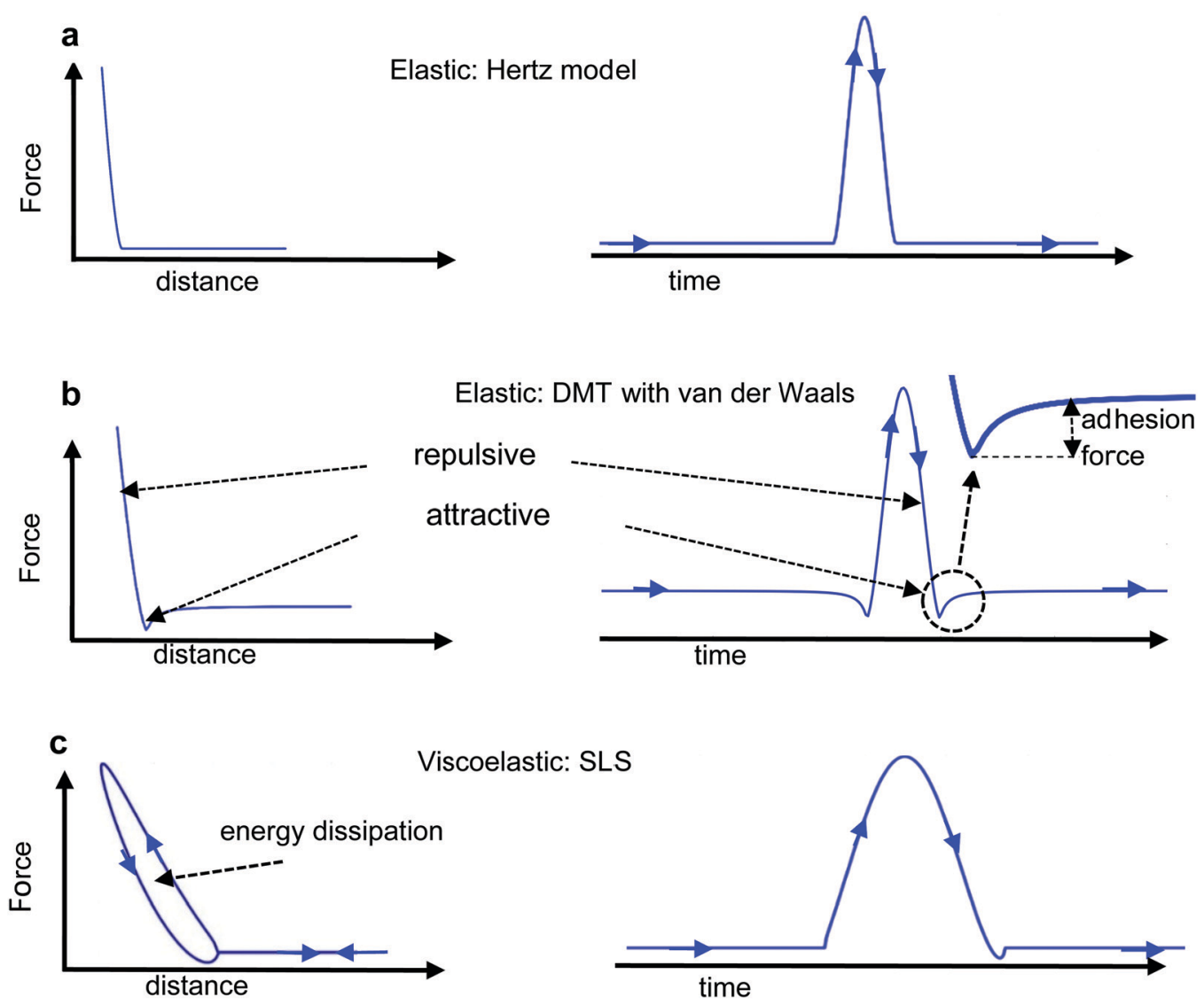

Fig. 3 tip-surface interaction forces. (a) Hertz model. The interaction is elastic and the force is repulsive upon contact. (b) DMT model. The interaction is elastic. Upon contact, the long-range van der Waals attractive force gives rise to the adhesion force. (c) SLS viscoelastic model. The interaction force depends on the direction of tip's motion. The interaction forces are shown as function of the distance and time.

Euler-Bernoulli equation can be approximated by the Newton equation for a point-mass system. ${ }^{35}$ Unless otherwise stated, the tip's displacement, oscillation or deflection refers to the one of the cantilever-tip system.

3.1.1 On-resonance equation. The equation of motion, for a tip that is excited by a driving force tuned at its first resonance or close to it, is the standard equation of amplitude modulation $\mathrm{AFM}^{31,37}$ (AM-AFM). Let's remind that tapping mode and amplitude modulation are different names for the same dynamic AFM method. ${ }^{31}$ The equation that describes AM-AFM is given by ${ }^{33,37,38}$

$$
F_{\text {ts }}(d(t))=k z+\frac{m \omega_{0}}{Q} \dot{z}+m \ddot{z}-F_{0} \cos \omega t
$$

the solution includes two terms, a transient and a steady-state solution. ${ }^{31}$ The transient term oscillates at the resonant frequency but decays exponentially as $\exp \left[-\left(\omega_{0} / 2 Q\right) t\right]$. The steady-state solution in the absence of higher harmonic components is given by

$$
z=z_{0}+A \cos (\omega t-\phi)
$$

where $F_{0}$ and $A$ are, respectively, the amplitudes of the driving force and tip's oscillation; $\omega(f=\omega / 2 \pi), \omega_{0}=(k / m)^{2}$ are, respectively, the angular frequencies of the driving force and the first resonance (fundamental mode); $\varphi$ is the phase shift between the driving force and the tip's response; $k$ and $Q$ are the force constant and quality factor of the cantilever-tip system (fundamental mode); $z$ and $z_{0}$ are, respectively, the instantaneous and the mean deflections. The effective mass $m$ is about $\frac{1}{4}$ the total mass of the cantilever-tip system. ${ }^{35}$ The cantilever deflection $z(t)$ is measured with respect to its rest (undeflected) position (Fig. 1e).

3.1.2 Off-resonance equation. If the tip is driven by a force tuned at a frequency far lower than its 1st resonant frequency (fundamental mode), it is convenient to replace the driving force in eqn (1) by a modulation term in the tip-sample distance, then ${ }^{27}$

$$
\begin{gathered}
F_{\mathrm{ts}}(d(t), \dot{d}(t))=k z+\frac{m \omega_{0}}{Q} \dot{z}+m \ddot{z} \\
d(t)=z_{\mathrm{c}}+z(t)+A_{\mathrm{m}} g\left(\omega_{\mathrm{m}} t\right)
\end{gathered}
$$

$d(t)$ is the instantaneous distance between tip and sample; $g(t)$ represents the waveform that modulates the tip-surface distance (triangular, sinusoidal or any other); $z_{\mathrm{c}}, A_{\mathrm{m}}$ and $\omega_{\mathrm{m}}$ are, respectively, the probe height, the amplitude and the angular frequency at which the distance is modulated (Fig. 1d). Eqn (3) indicates that, in general, $F_{\text {ts }}$ does not coincide with $k z$. The implications of this result to measure forces are described in Section 5. 

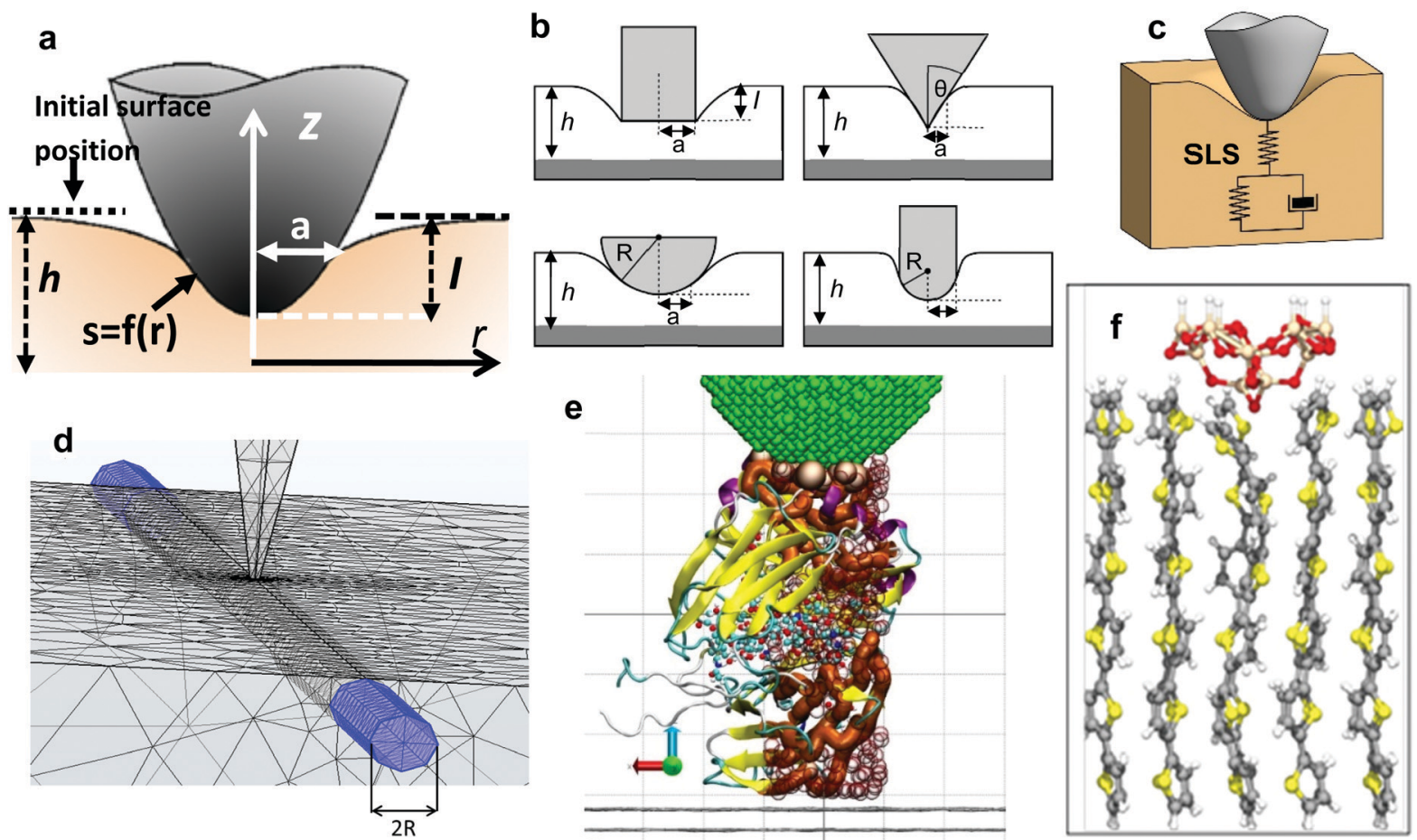

Fig. 4 Models and simulations of tip-soft material interfaces. (a) Definition of the main interfacial parameters; $a$ is the radius of the projected contact area; $h$ is the sample thickness; $r$ is the radial coordinate; $f(r)$ is the shape of the contact. (b) Axisymmetric tip geometries. From left to right and top to bottom, cylinder, cone, sphere and nanowire. (c) Scheme of a tip-linear viscoelastic material (SLS) interface. (d) FEM simulation of the deformation of a fiber embedded in a soft matrix. Adapted with permission from ref. 67. Copyright 2019 American Chemical Society. (e) MD simulation of the deformation of an IgG antibody domain by a carbon nanotube tip. Image provided by R. Perez and J. G. Vilhena. (f) Ab initio MD simulations of the deformation induced on a sexithiophene chain by a silica tip. The force produces the bending of the head molecular groups and its transmission through the whole chain. Color code for atoms: oxygen (red), sulphur (yellow), carbon (grey), hydrogen (white) and silicon (light brown). Reprinted with permission from ref. 78. Copyright 2009 Institute of Physics.

\section{Theory of nanomechanical force spectroscopy: contact mechanics models}

The mechanical properties measured by an AFM are usually explained in terms of continuum mechanics models (Fig. 4a and b). In general, the interaction force has conservative or elastic $F_{\text {con }}$ and non-conservative or inelastic $F_{\text {dis }}$ contributions. ${ }^{39}$ The total work involved in the deformation of a material can be separated into the energy stored elastically in the deformation of the material and the energy dissipated as heat through viscous losses or surface adhesion hysteresis. Then, as a first approximation the force can be decomposed into ${ }^{39,40}$

$$
F_{\mathrm{ts}}=F_{\text {con }}+F_{\text {dis }}
$$

\subsection{Elastic tip-sample deformations}

To determine the deformation produced by an elastic tip on an elastic sample the first is to establish the constitutive equation between the stress and the strain. For a linear, homogenous and isotropic elastic material, the stress-strain equation is given by ${ }^{41-43}$

$$
\sigma_{i j}(t)=\frac{E}{3(1-2 \nu)} u_{l l}(t) \delta_{i j}+\frac{E}{1+\nu}\left(u_{i j}(t)-\frac{1}{3} u_{l l}(t) \delta_{i j}\right)
$$

where $\sigma_{i j}(t)$ is the stress tensor, $u_{i j}(t)$ is the strain tensor and $\delta_{i j}$ is Kronecker's delta; $u_{l l}$ indicates the trace of the strain tensor. The material (sample) is described by a set of two independent parameters, such as the Young's (elastic) modulus $E$ and the Poisson ratio $\nu$. Any elastic constant can be expressed as a function of any two others. Therefore, the constitutive expression could also be expressed in terms of the bulk modulus $K$ and the shear modulus $G .^{42}$

For a tip-sample interface is common to introduce the effective elastic modulus (also called reduced elastic modulus) $E_{\text {eff. }}$

$$
\frac{1}{E_{\mathrm{eff}}}=\frac{1-\nu^{2}}{E}+\frac{1-\nu_{\mathrm{t}}^{2}}{E_{\mathrm{t}}} \approx \frac{1-\nu^{2}}{E}
$$

where the subscript $t$ indicates the elastic constants of the tip. The effective elastic modulus takes into account the fact that both the sample and the tip have finite elastic constants. Therefore, elastic deformations occur both in the sample and the tip and the total deformation the sum of the sample and the tip's deformations; ${ }^{47}$ however, for soft materials, $E \ll E_{t}$.

To apply eqn (6) to AFM data requires to introduce a set of boundary conditions about the geometry of the interface and the spatial components of the stress. For example, the contact region between the tip and the sample must coincide with the 
shape of the tip (Fig. 4a). However, it is beyond the scope of this review to introduce the relevant boundary conditions and their mathematical implications. That information can be found elsewhere. $^{44}$ The boundary conditions enable to transform the stress-strain constitutive equation into a force-indentation integral that only involves the vertical component of the stress. Some analytical solutions of this integral were developed by Hertz and Boussinesq in the late XIX century, ${ }^{43,45}$ this is, well before the AFM was invented. The formalism developed by Sneddon $^{46}$ is valid for any axisymmetric tip acting on a semiinfinite elastic material, in particular, for a flat cylinder, a cone and a paraboloid of revolution. The latter solution converges to Hertz model for a half-sphere. ${ }^{43,46}$

Sneddon's results can be formulated into a compact expression of the force as a function of the indentation $I$ for any axisymmetric tip

$$
F_{\mathrm{ts}}(I)=\alpha E I_{\beta}
$$

where $\alpha$ is a parameter that depends on the tip's geometry and the Poisson's ratio and $\beta$ is a parameter that depends on the tip's geometry.

Sneddon theory implies several assumptions. Namely, (1) the deformation in is the elastic regime; (2) the deformation is small with respect to the radius of the projected contact area; (3) the force is applied perpendicular to the surface, this is, there are not shear forces. and (4) the sample is semi-infinite.

Tables 1 and 2 show, respectively, the coefficients needed to calculate the force as a function of the indentation and the contact radius for the tip-sample geometries shown in Fig. $4 \mathrm{~b}$. The contact radius is calculated for semi-infinite and a layer of thickness $h$ (see Section 5). In Table 2 the coefficient $\alpha_{0}=-1.7795$ is obtained for a layer which is fixed to a rigid support. Table 3 shows the definitions of the most common quantities in nanomechanical mapping.

\subsection{Inelastic tip-surface deformations}

An inelastic deformation is associated with a non-conservative interaction. In AFM, a non-conservative force implies with some loss of the tip's energy. ${ }^{39}$ Non-conservative interactions are generated by a variety of processes that might depend on the material properties, the tip and/or the environment. For example, non-conservative interactions are involved in forceinduced atomic or molecular reorientations, rupture of ligandreceptor bonds, interdigitation, the formation of a liquid more generally, in any exchange of atoms and molecules between the tip and the sample surface.

Table 1 Coefficients to determine the force

\begin{tabular}{lcc}
\hline Tip's geometry & $\alpha$ & $\beta$ \\
\hline Cylinder & $\frac{2 a}{\left(1-\nu^{2}\right)}$ & 1 \\
Cone & $\frac{2 \tan \theta}{\pi\left(1-\nu^{2}\right)}$ & 2 \\
Half-sphere & $\frac{4 \sqrt{a}}{3\left(1-\nu^{2}\right)}$ & 1.5
\end{tabular}

Table 2 Radius of the projected area of contact $\left(A=\pi a^{2}\right)$

\begin{tabular}{lll}
\hline Tip's geometry & $\begin{array}{l}\text { Contact radius } \\
\text { (semi-infinite system) }\end{array}$ & $\begin{array}{l}\text { Contact radius } \\
\text { (layer of thickness } h \text { ) }\end{array}$ \\
\hline $\begin{array}{l}\text { Cylinder of radius } R \\
\text { Cone (half-angle } \theta \text { ) }\end{array}$ & $\frac{2 \tan \theta}{\pi} I$ & $R$ \\
$\begin{array}{l}\text { Half-sphere of } \\
\text { radius } R\end{array}$ & $\sqrt{R I}$ & $\frac{2 \tan \theta}{\pi} I\left[1-\frac{2 \alpha_{0} \tan \theta}{\pi^{2} h} I\right]$ \\
& & $\sqrt{R I}\left[1-\frac{2 \alpha_{0} \sqrt{R I}}{3 \pi h}\right]$. \\
\hline
\end{tabular}

Four processes either acting individually or simultaneously dominate the non-conservative interactions measured in a tip-soft material interface: velocity dependent processes and viscoelasticity, rupture of molecular bonds, surface adhesion hysteresis and mass exchange between tip and sample.

Some of the tip's energy is also lost by displacement of the surrounding fluid (air, gas or liquid) during the tip's motion. This gives rise an extended hydrodynamic damping process which is proportional to the velocity and scales with the inverse of the quality factor. Each mode has a distinctive spatial shape; therefore, the hydrodynamic damping depends on the mode. ${ }^{20}$ This process is unrelated to the sample's mechanical properties. Nonetheless, it must be taken into account to balance the total energy in an oscillation cycle. ${ }^{31}$

4.2.1 Viscoelastic interactions and models.. Many soft materials are viscoelastic, this is, they show a mechanical response that combines the elastic response of a solid and the viscous flow of a liquid. A distinctive feature of a viscoelastic material is that the response to a mechanical force or deformation is not instantaneous. In many cases, a viscoelastic process shows a dependence on the velocity and the history of the deformation.

The development of a viscoelastic description of the response of a material based on AFM data can be divided in two main steps. First, it requires a viscoelastic model. This can be achieved either by using mechanical-equivalents or analogues based on a combination of spring and dashpots elements (Fig. 4c) or by using continuum mechanics theories. The second step requires the determination of the interaction force from the observables.

The viscoelastic correspondence principle ${ }^{48}$ simplifies the theoretical effort of expressing the tensorial stress-strain equation of a viscoelastic material into an equation of the force as a function of the indentation. This principle transforms the elastic parameters into relaxation functions. It can be shown that for an incompressible material $(\nu=0.5)$ the force is ${ }^{49}$

$$
F(I(t), t)=\alpha \int_{0}^{t} \psi_{E}\left(t-t^{\prime}\right) \frac{\mathrm{d}}{\mathrm{d} t^{\prime}}\left(I\left(t^{\prime}\right)^{\beta}\right) \mathrm{d} t^{\prime}
$$

where the elastic modulus $E$ has been replaced by an operator that contains the compressive relaxation function $\psi_{\mathrm{E}}(t)$. Eqn (9) shows that the force is a function of time, the indentation history, and the relaxation function of the material. To solve the above equation requires an explicit expression for the relaxation function.

4.2.2 Three-dimensional Kelvin-Voigt model. Linear viscoelastic models facilitate the qualitative and semi-quantitative 
Table 3 Common quantities in nanomechanical mapping

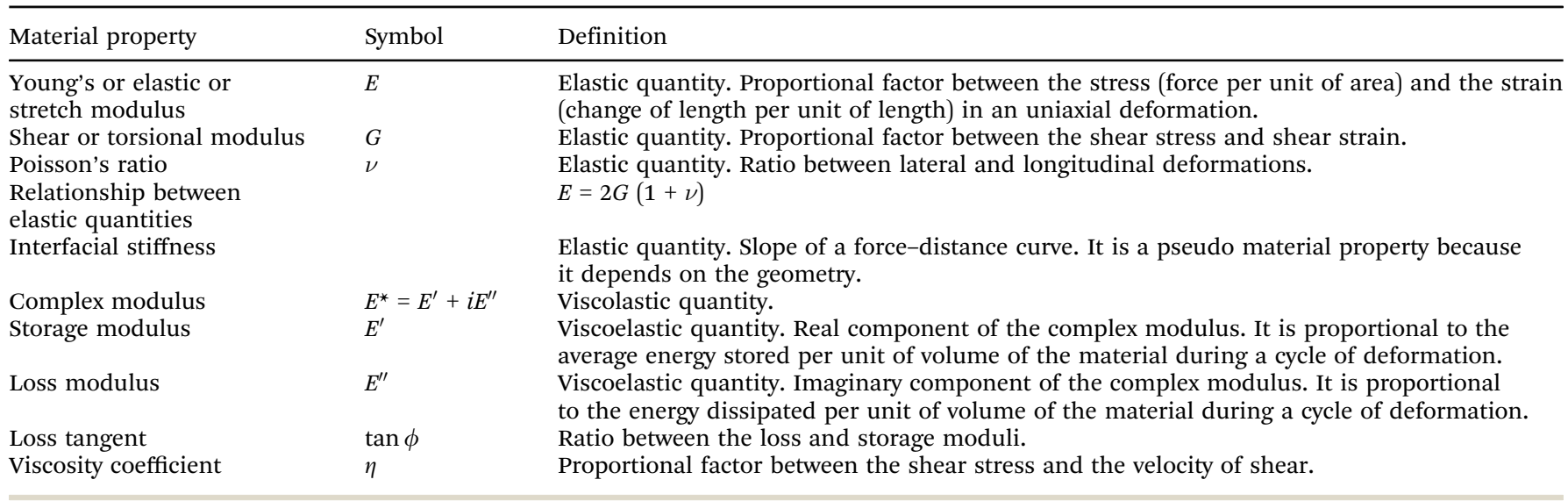

understanding of the viscoelastic response of many of the materials. ${ }^{42}$ The Kelvin-Voigt (KV) model combines a linear elastic spring in parallel with a linear dashpot (viscous element). ${ }^{42}$ Its relaxation function is given by

$$
\Psi_{\mathrm{KV}}(t)=E+3 \eta \delta(t)
$$

where $\delta(t)$ is the Dirac delta function; $\eta$ is the shear viscosity coefficient; $\eta$ is related to the compression or Trouton's coefficient of viscosity by $\eta_{E}=3 \eta .{ }^{49}$

The application of the KV model to AFM requires introducing the geometry of the tip and an estimation of the contact area. This has been accomplished by using Hertz contact mechanics. This is the basis of the 3D-KV model, which is obtained by solving en 9 with the relaxation function (eqn (10)), then ${ }^{49}$

$$
F(I, t)=\alpha I(t)^{\beta-1}[E I(t)+3 \beta \eta \dot{I}(t)]
$$

which for a sphere of radius $R$ gives (see Table 1 )

$$
F_{\mathrm{ts}}(I, \mathrm{~d} I / \mathrm{d} t)=\frac{4}{3} \frac{E}{1-\nu^{2}} \sqrt{R} I^{3 / 2}+\frac{6}{1-\nu^{2}} \sqrt{R I} \eta \dot{I}
$$

4.2.3 Standard linear solid model (SLS). The onedimensional representation of the SLS model ${ }^{42,50}$ is made of a spring either in series with a Kelvin-Voigt element or in parallel with a Maxwell element. The relaxation function is given by

$$
\Psi_{\mathrm{SLS}}(t)=E_{1}+E_{2} \exp \left(-\frac{E_{2} t}{3 \eta}\right)
$$

where $E_{1}$ and $E_{2}$ are the elastic's moduli, respectively, at low and high frequencies. For this model there is not a closed-form analytical solution for the dependence of the force with the indentation.

4.2.4 Three-dimensional power-law rheology. The relaxation function of a power-law rheology model $^{51}$ is given by

$$
\Psi_{\mathrm{PLR}}=E_{0}\left(\frac{t}{t_{0}}\right)^{-\chi}
$$

where $E_{0}$ is the apparent elastic modulus at time $t_{0}, \chi$ is the power-law exponent and $t_{0}$ is a timescale factor which is set to $1 \mathrm{~s} .^{51}$ The exponent describes the viscoelastic behavior of the material. It takes values between $\chi=0$ (elastic material) and $\chi=1$ (Newtonian liquid).

For a conical tip of half-angle $\theta$ and a tip's displacement modulated by a waveform, it is deduced ${ }^{52}$

$$
F(t)=\frac{8 \tan \theta}{3 \pi} 2 v^{2} t^{2}\left(\frac{t}{t_{0}}\right)^{-\chi} \frac{\Gamma[1-\chi]}{\Gamma[3-\chi]}
$$

where $\Gamma$ is the gamma function and $v$ the tip's velocity.

4.2.5 Surface adhesion hysteresis. Surface adhesion hysteresis implies that work needed to separate two surfaces is always greater than the originally gained by bringing the surfaces together. ${ }^{45}$ This process implies that the surface energy of the system $\gamma$ has two different values, one for the approach $\gamma_{\mathrm{a}}$ and the other the retraction $\gamma_{\mathrm{r}}$. In the framework of the DerjaguinMuller-Toporov theory, ${ }^{53}$ the interaction force including adhesion is

$$
F(I)=\frac{4}{3} \frac{E}{1-\nu^{2}} \sqrt{R} I^{3 / 2}-4 \pi R \gamma
$$

\subsection{Relationship between deflection, distance and indentation}

The above analytical equations express the force as a function of the indentation (deformation) produced by the tip. However, the main AFM observable is the tip's deflection. Therefore, some additional eqns are needed to transform the deflection into instantaneous tip-sample distances and indentations. The indentation is defined as

$$
I=-d(t) \text { for } d \leq 0
$$

where $d$ is the instantaneous tip-sample distance. In offresonance experiments (Fig. 1d) the instantaneous distance is determined by eqn (3). In an on-resonance experiment, the instantaneous distance is given by (Fig. 1e)

$$
d(t)=z_{\mathrm{c}}+z(t)+A \cos (\omega t-\phi)
$$

The determination of the contact point between the tip and the sample demands some special considerations when imaging very soft materials such as cells. ${ }^{54}$ 


\subsection{Simulations}

Analytical solutions of the integral equation eqn (9) are available for a few combinations of viscoelastic models, distance modulation waveforms and tip geometries. This limitation has motivated the introduction of the computational methods to describe in a self-consistent manner the tip-sample viscoelastic interactions and the associated deformations. ${ }^{55,56}$

Computational solutions enable to explore the dynamic behaviour of complex models. This task is helped by the existence of two numerical codes dForce ${ }^{57}$ and VEDA $^{58}$ that enable to simulate the response of a dynamic AFM for a variety of sample models. Numerical simulations have been applied to determine the force based on a SLS response and to compare it with experiments performed with an amplitude modulation AFM on elastomers and polycarbonate samples. ${ }^{56}$

4.4.1 Finite element method simulations. Finite element method (FEM $)^{59}$ is very useful to visualize the deformations generated by the tip on a variety of elastic and viscoelastic models. The simulations facilitate the understanding of the role the tip geometry on the mechanical response of a material. FEM simulations are also useful to test the theoretical models proposed to determine the force exerted on an AFM experiment. In those comparisons, the forces given by the FEM simulations are considered to provide the true behavior of the viscoelastic material.

In a FEM simulation, the tip-sample interface is discretized in mesh units (Fig. 4d). The accuracy of the simulations depends on the number of mesh units. Ideally, for a highspatial resolution mechanical measurement small mesh units of 1-2 $\mathrm{nm}$ in length should be used. In addition, the tip radius must be several times larger than the maximum indentation to avoid convergence problems. FEM simulations were used to understand AFM experiments performed on cells ${ }^{60-67}$ and to describe the elastic response of a virus. ${ }^{68,69}$

4.4.2 Molecular dynamics simulations. Molecular dynamics (MD) and atomistic simulations have been extensively applied to explain atomic-scale resolution images obtained by scanning probe microscopy methods. ${ }^{70-77}$ MD simulations have also been used to test the equations of continuum contact mechanics in nanometer scale contacts. ${ }^{74}$ Large-scale molecular dynamics (MD) simulations have also been applied to study the adsorption of proteins on surfaces. ${ }^{75}$ Those studies provide information about preferential adsorption sites and insight into the biomoleculesurface interactions and their effect on the apparent topography of the biomolecule. ${ }^{76}$

In the context of nanomechanical force spectroscopy, MD simulations are helpful to address three relevant aspects. First, to explain the mechanical response of a biomolecule under the deformation exerted by the tip (Fig. 4e and f). ${ }^{76,77}$ Second, to understand the intramolecular mechanisms of energy dissipation. ${ }^{78}$ Finally, to assess the capability of AFM to distinguish between the mechanical response of the different domains or subunits of a protein.

It should be noted that the time-scales of MD simulations (0.1-10 ns) and AFM experiments (0.1-100 ms) are still separated by a few orders of magnitude. This gap might set some limitations in the comparisons between measurements and MD simulations.

\section{Theory of nanomechanical force spectroscopy: living cells}

Living cells, in particular, eukaryotic cells pose some challenges to the imaging and mapping capabilities of a force microscope. Currently, the best images of eukaryotic cells obtained by AFM have a spatial resolution in the $100 \mathrm{~nm}$ range. ${ }^{11,67,79-81}$ Those values are modest if they are compared with the spatial resolution achieved on other biological systems such proteins $(\sim 1 \mathrm{~nm})^{24}$ or protein fibers. ${ }^{82}$ Mapping with accuracy the elastic modulus of a cell is also challenging because several factors contribute to the error in the measurement. ${ }^{44,83}$ To address and eventually overcome those issues requires understanding the role of the main factors that control the imaging and mapping of the cell's mechanical properties.

\subsection{Softness, viscoelasticity, finite-thickness and incompressibility}

Living eukaryotic cells are among the softest materials on earth. Some mammalian cells show an elastic modulus value below $1 \mathrm{kPa}$ range. ${ }^{84,85}$ In general, an eukaryotic cell is about 4 orders the magnitude softer than a single protein $(\sim 50 \mathrm{MPa}){ }^{24}$ six orders of magnitude softer than polystyrene $(\sim 2 \mathrm{GPa})^{86}$ and about eight orders magnitude softer than silicon $(\sim 150 \mathrm{GPa}) .{ }^{87}$ Under the same applied force (say $300 \mathrm{pN}$ ), the best AFM image of a eukaryotic cell has a spatial resolution of about $100 \mathrm{~nm}$, this is, about 3 orders of magnitude worse than the one obtained on a rigid crystalline surface $(\sim 0.1 \mathrm{~nm})$.

An eukaryotic cell might be considered as a solid system made by plasma membrane that encloses a variety of solid elements such as single proteins, molecular motors, protein filaments, DNA, organelles and a nucleus immersed in an aqueous solution. A moving solid element in a liquid involves viscoelastic interactions. Viscoelasticity is an intrinsic feature of the mechanical response of a cell, ${ }^{51,84,88,89}$ therefore AFMbased measurements should always be described in terms of viscoelastic models. ${ }^{49,52,90-97}$

A single cell is a heterogeneous, anisotropic and finitethickness material (Fig. 5a and b). Currently, there is not a well-accepted contact mechanics model that captures the structural complexity of an eukaryotic cell. ${ }^{84}$ In addition, the theory to explain how the cell's mechanical properties influence the cantilever's deflection and the tip's indentation is just emerging. ${ }^{52,93,96}$

Modeling the mechanical response of a cell is greatly simplified by considering that cells are incompressible $(\nu=0.5)$ and are locally isotropic. The first assumption is supported by the mass densities of HeLa cells and fibroblasts which are, respectively, 1.08 and $1.14 \mathrm{~g} \mathrm{~cm}^{-3}$. Those values are close to the water mass density $\left(1 \mathrm{~g} \mathrm{~cm}^{-3}\right)$. The mass of the above cells was obtained from micromechanical resonator experiments. ${ }^{98}$ The second assumption implies that the cell 

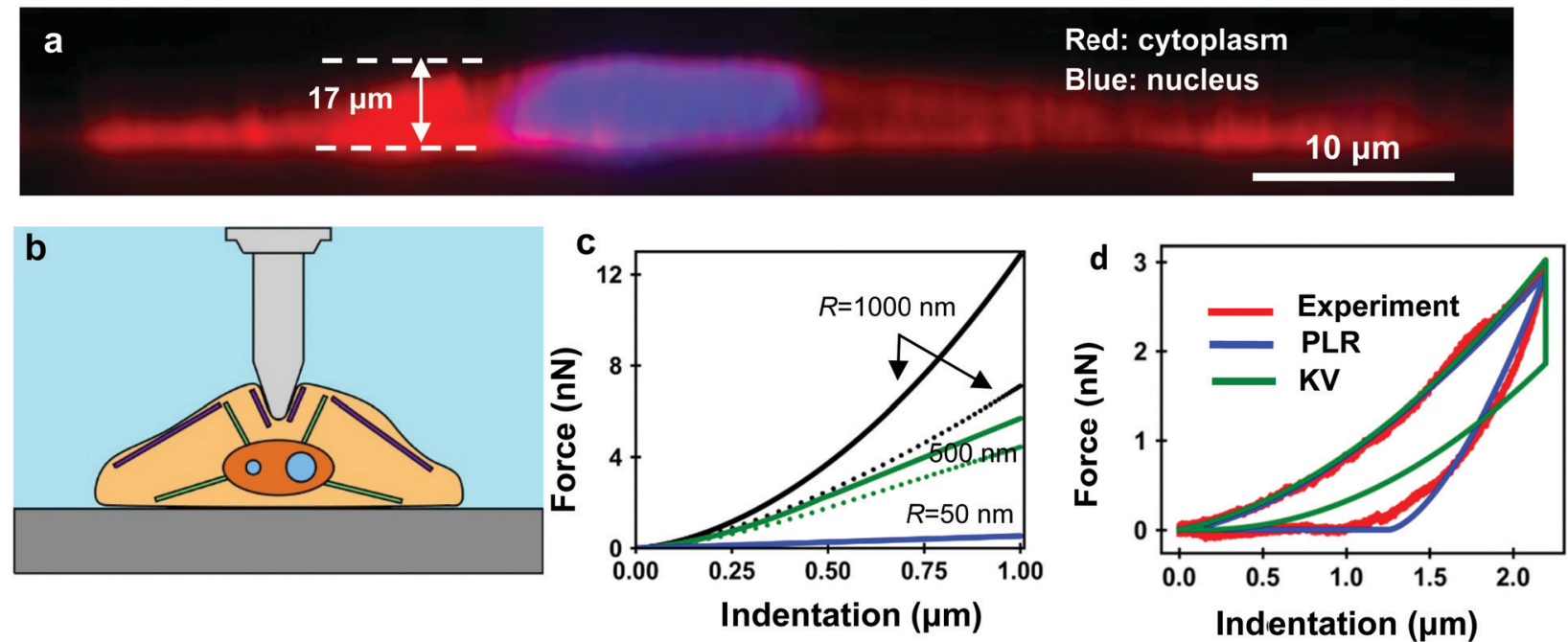

Fig. 5 (a) Optical microscope side-view of a cardiomyocyte cultured on a Petri dish. The red signal comes from the fluorophores attached to actin fibers while the blue signal comes from the fluorophores inside the nucleus. Image provided by C. Marquez-Lopez and J. A. Bernal. (b) Scheme of a living cell-tip interface in a liquid environment. The cell thickness depends on the lateral position. (c) Force-indentation curve for nanowires of different $R$. The discontinuous lines show the force curves produced by the same probe on a semi-infinite sample. For $R=50 \mathrm{~nm}$, the force curves given by the semiinfinite model and the bottom effect elastic theory are nearly identical. Layer, $E=4 \mathrm{kPa}, h=2.5 \mu \mathrm{m}$; semi-infinite material $E=4 \mathrm{kPa}$. Adapted with permission from 44. Copyright 2018 Elsevier. (d) Experimental (red) and theoretical force-distance curves (blue and green) obtained on a living NIH 3T3 fibroblasts. Reprinted with permission from ref. 52. Copyright 2020 Royal Society of Chemistry.

membrane and cytoskeleton deform axisymmetrically when indented by axisymmetric tips. To what extent this assumption captures the local properties measured by AFM experiments is currently under debate. ${ }^{11,66}$ The heterogeneity of a cell is captured by considering it as a layered system (membrane, cortex, cytoplasm and nucleus). This approximation simplifies and facilitates FEM simulations. ${ }^{60,66,67}$

\subsection{Bottom-effect correction for an elastic and fine-thickness layer}

The softness, finite thickness and incompressibility of an adherent cell imply that the force exerted by the tip on the cell is influenced by the stiffness of the solid support. This effect is unavoidable. It implies that any compressive force measurement on an adherent cell bears the influence of the solid support (Fig. 5c). This observation makes semi-infinite contact mechanics models such as Sneddon or Hertz unsuitable to describe quantitatively the forces, deformations and mechanical properties of a cell.

Bottom-effect correction theories aim to determine the true Young's modulus of a very soft material deposited on a rigid support from a force-distance curve. ${ }^{44,65,99}$ Those theories for elastic and finite-thickness materials are firmly established. They have been validated by finite element model simulations. ${ }^{44,65}$ In addition, FEM simulations show the need of using bottom-effect corrections. Some simulations showed that the elastic modulus value deduced by fitting a FDC curve to a semi-infinite model could be off by a factor 2 to 10 from the expected value. ${ }^{44}$

Chadwick and co-workers proposed the first bottom-effect correction expression for the force exerted by a spherical probe on a finite-thickness elastic layer. ${ }^{99}$ A more general theory that is valid for any axisymmetric tip was developed by Garcia and Garcia. ${ }^{44}$
Ros and co-workers have extended the bottom-effect correction for the case of cells deposited on a soft matrix. ${ }^{65}$

The force exerted by an axisymmetric probe on an elastic and finite-thickness layer deposited on rigid support can be expressed as a sum of the force exerted on a semi-infinite system with the same elastic parameters than the layer and a correction term that accounts for the finite-thickness the is given by $^{44}$

$$
F=F_{\text {semi-infinite }}+F_{\text {correction }}
$$

In particular for a cone of half-angle $\theta$ the force is given by ${ }^{44}$

$$
F(I)=\frac{8 \tan \theta}{3 \pi} E I^{2}+0.721 \frac{8 \tan ^{2} \theta}{3 \pi h} E I^{3}+0.650 \frac{8 \tan ^{3} \theta}{3 \pi h^{2}} E I^{4}+O\left(\frac{I^{5}}{h^{3}}\right)
$$

Eqn (20) reveals that the force scales with the projected contact radius (see Table 2) and the sample thickness $h$. For the same thickness, the effect of the solid support on the apparent viscoelastic parameters decreases by decreasing the tip's half angle. This result indicates that sharper tips should be used to measure nanomechanical properties of cells.

Fig. $5 \mathrm{c}$ shows the competition between the bottom effect, the indentation and the contact area for the force exerted by a nanowire tip on a soft elastic layer $(E=4 \mathrm{kPa} ; h=2.5 \mu \mathrm{m})$ deposited on a rigid support. ${ }^{44}$ To visualize the bottom effect contribution we have calculated the force exerted by the same probe on a semi-infinite sample (discontinuous curves). The bottom effect plays a major factor in the determination of the force whenever the contact radius and sample thickness are within the same order of magnitude. The implication for cell nanomechanics is that the use of sharp and high-aspect ratio 
probes significantly reduces the influence of the solid support in the determination of the force. This result might be counterintuitive. It challenges some earlier recommendations to perform elastic modulus measurements on cells. ${ }^{64,83}$ An earlier bottom-effect correction for the force exerted by a conical tip on an elastic material ${ }^{100}$ does neither agree with eqn (20) nor with FEM simulations. ${ }^{44,65}$

It has been proposed that the surface tension of cells could explain the apparent increase of the elastic modulus of an adherent cell. ${ }^{101}$ This hypothesis is not supported by experimental data. It is also in conflict with the bottom-effect theory introduced above and FEM simulations. ${ }^{44,65}$

\subsection{Bottom-effect correction for a viscoelastic and fine- thickness layer}

An eukaryotic cells is a finite-thickness viscoelastic material. Therefore, bottom-effect elastic corrections introduced in the previous section sever as the starting expressions to develop their viscoelastic counterparts. A viscoelastic response introduces a dependence on the deformation history. In other words, the contact area for the same deformation will depend on whether the tip is approaching or withdrawing from the sample surface.

Analytical bottom-effect viscoelastic corrections have been deduced for some viscoelastic models. ${ }^{52}$ The theoretical approach combines bottom-effect elastic corrections, ${ }^{44}$ the correspondence principle between elastic and viscoelastic deformations, ${ }^{48}$ and Ting's method to determine the force as a function of the deformation history. ${ }^{102}$

In particular, for a power-law rheology model, ${ }^{52}$ the force for the tip approaching towards the cell is given by (Fig. 5c),

$$
\begin{aligned}
F(t)= & \frac{16 \tan \theta}{3 \pi} v^{2} t^{2}\left(\frac{t}{t_{0}}\right)^{-\chi} \frac{\Gamma[1-\chi]}{\Gamma[3-\chi]} \\
& +0.721 \frac{16 \tan ^{2} \theta}{\pi h} v^{3} t^{3}\left(\frac{t}{t_{0}}\right)^{-\chi} \frac{\Gamma[1-\chi]}{\Gamma[4-\chi]}+O\left[\frac{v^{4} t^{4}}{h^{2}}\right]
\end{aligned}
$$

where $\Gamma$ is the gamma function and $O$ is a function that includes the higher order correction terms. The forces deduced from eqn (21) were compared to FDCs obtained on living fibroblast cells (Fig. 5d). ${ }^{53}$ The agreement obtained between experiments and eqn (21) validates the theory and the powerlaw rheology model to describe the response of fibroblasts to compressive forces.

\section{Force-volume}

Force-volume (FV) is widely used to measure mechanical properties at the nanoscale. It is a feature included in the applications modules of many commercial AFMs. ${ }^{28} \mathrm{~A}$ forcevolume map combines a topographic image of the surface with the acquisition of one (or several) force-distance curves on each point (pixel) of the surface (Fig. 6a). FV has experienced significant changes in the last few years, however, some of the features enunciated by Hasnma and co-workers ${ }^{14,15}$ about
25 years ago and later conceptualized by the schemes provided by Heinz and $\mathrm{Hoh}^{4}$ remain unchanged.

The first implementations of force-volume used a triangular waveform to modulate the tip-surface distance. ${ }^{14}$ Triangular waveforms are still very common because they offer a straightforward relation between distance and time $(d=v t)$. However, they might introduce some side-effects. First, a triangular waveform contains a large number of higher harmonics. Higher harmonics could be coupled to some of the mechanical resonances of the AFM. This coupling might complicate the interpretation of the data. Second, the velocity has a discontinuity at the turning point which might introduce some artefacts at moderate or high frequencies. Those issues motivated the introduction of sinusoidal waveforms. ${ }^{103-106}$

Two recent developments enhanced the application capabilities of force-volume. First, the determination of rheological properties by processing spatially-resolved $\mathrm{FDCs}^{67,107,108}$ is poised to have a significant impact in the determination of viscoelastic properties at the nanoscale. Second, the use of biofunctionalized tips has opened new applications in mechanobiology and sensing. ${ }^{109,110}$

\subsection{Force-volume methodology}

Force-volume is an off-resonance AFM method. It provides arrays of spatially-resolved FDCs (Fig. 6a). A force-distance curve is acquired by recording the cantilever deflection as a function of the relative tip-surface distance. Initially, the tip and the sample surface are far apart which implies that the interaction force is negligible. Then, the tip (surface) is continuously displaced towards the surface (tip) until the force reaches a pre-fixed value.

A force-distance curve includes two sections, approach and retraction (Fig. 6b). Each section includes regions dominated by long-range attractive and regions dominated by short-range repulsive forces (Fig. 3b). The approach and retraction sections of the FDC might not coincide (force hysteresis). In fact, the presence of hysteresis in a FDCs is very common. Force-distance curve hysteresis implies energy dissipation. In a region where tip and sample are in contact, and in the absence of mass exchange between tip and surface, viscoelastic processes in the sample dominate the observed hysteresis in FDCs. Out of a contact region, the hysteresis is dominated by changes in the adhesion force, approaching versus retraction. Those changes might be associated with the presence of either specific (ligand-receptor bonds) or unspecific interactions.

Different types of waveforms can be used to modulate the tip-surface separation (sinusoidal, triangular or a combination of both) (Fig. 6c). To improve the signal-to-noise ratio, the FDC associated with a pixel might be the result of averaging several FDCs taken on the same point of the surface.

The transformation of cantilever deflections into interaction force values is performed by applying the postulate of force spectroscopy. This postulate establishes that the interaction force coincides with the cantilever deflection $z(t)$ times the cantilever static force constant $k$

$$
F_{\mathrm{ts}}(t)=k z(t)
$$


a
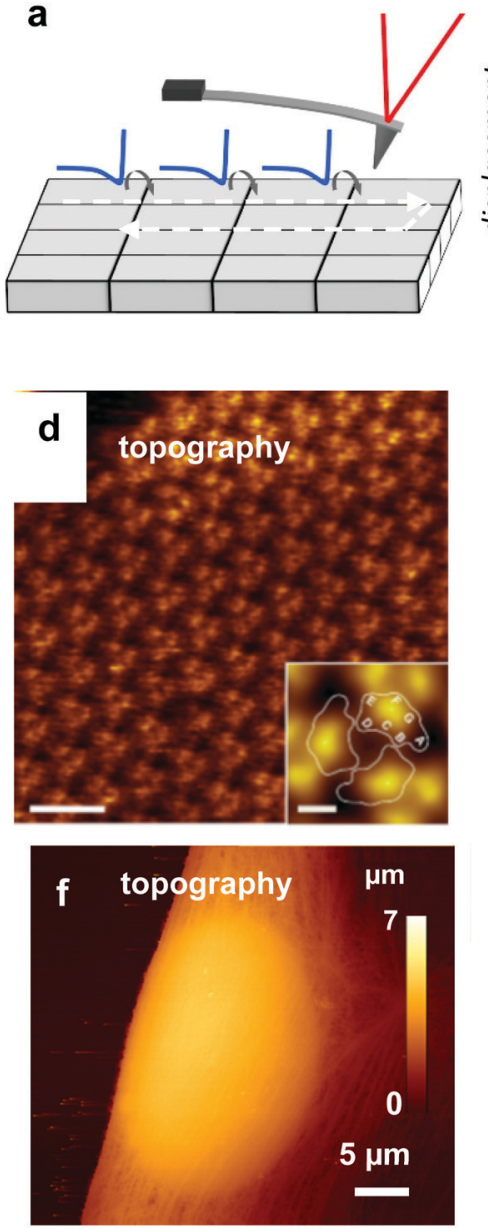

b
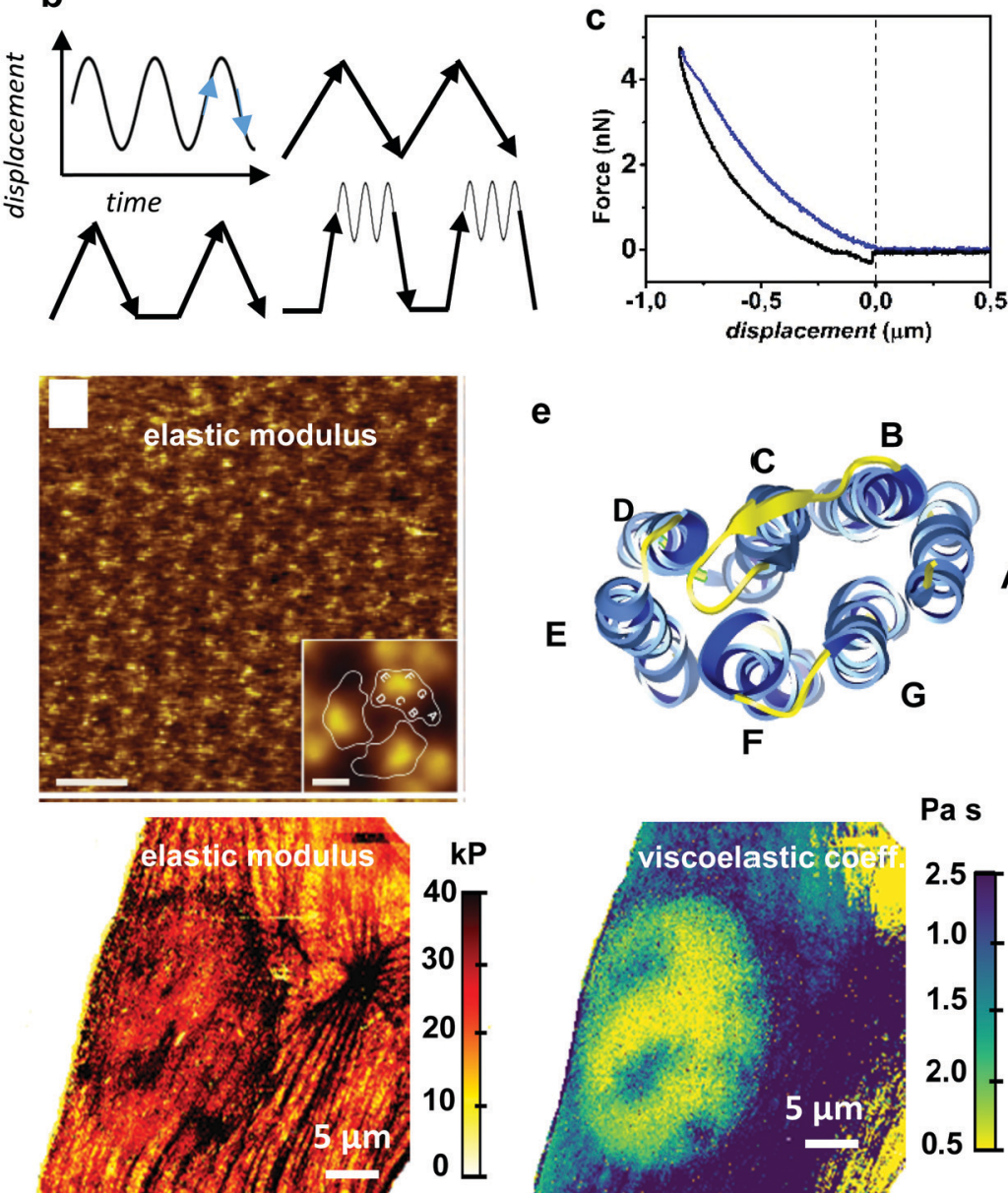

e

E

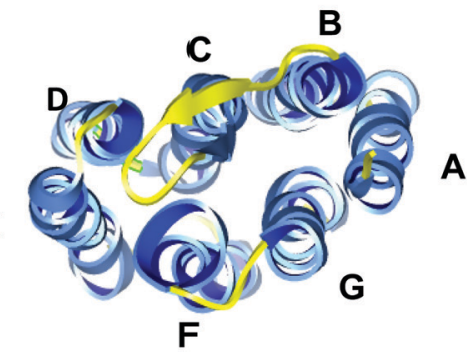

Pas
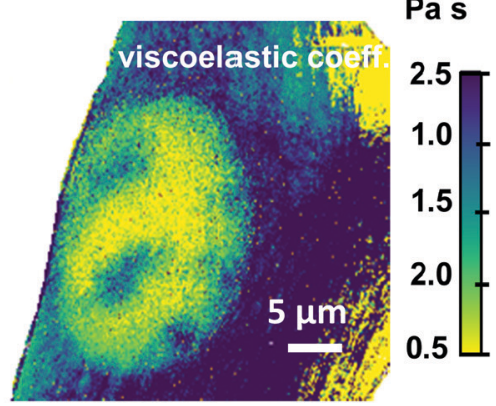

Fig. 6 Force-volume. (a) Scheme of FV mapping. (b) Different waveforms might be used to modulate the tip-sample displacement. The most common are the sinusoidal and the triangular. Some waveforms might include dwell times or pre-loading steps. (c) FDC obtained on a fibroblast cell. (d) From left to right, topography and elastic modulus of the cytoplasmic purple membrane side obtained by using a peak force of $75 \mathrm{pN}$. Insets show three-fold symmetrized averages of the bacteriodophsin (BR) trimer. Scale bar $10 \mathrm{~nm}$. Inset, scale bar $2 \mathrm{~nm}$. Reprinted with permission from ref. 111 . Copyright 2011 John Wiley and Sons. (e) BR protein. The seven transmembrane $\alpha$-helices A-G are indicated. Protein data bank code 2at9. (f) Topography, elastic and viscosity coefficient maps of a fibroblast cell. The Young's modulus map provide high-spatial resolution details of the outer cell structures such as the actin cross-linked networks (cortex) and the stress fibers. The viscosity coefficient map detects the presence of intracellular and subsurface structures (nucleoli). Reprinted with permission from ref. 67. Copyright 2011 American Chemical Society.

Several parameters can be extracted from a FDC. The most common are height, Young's modulus, stiffness, adhesion force and energy dissipation. By plotting each those parameters as a function of the spatial coordinate a multiparametric description of the interface is achieved ${ }^{67,111}$ (Fig. 6d and e). The value of the adhesion force is obtained by measuring the sudden changes in the deflection during the tip's withdrawal from the sample surface (Fig. 3b). The stiffness is directly obtained by measuring the slope of the force-distance curve in the repulsive region. The determination of the Young's modulus requires to fit a section of the FDC dominated by repulsive forces with a contact mechanics model. The determination of viscoelastic properties requires a theoretical model to associate observables with parameters (see below).

\subsection{The force-volume family}

Force-volume is implemented in the hardware and software of many commercial AFMs. Improvements in signal processing, closed-loops, software, data analysis and the addition of new parameters have led to a variety of denominations. ${ }^{28}$ The first commercial FV configuration that incorporated a sinusoidal waveform became very popular. ${ }^{106}$ It presented some advantages in the realization and/or interpretation of some experiments. However, neither eqn (3) nor the scheme shown in Fig. 6a was modified by using a sinusoidal waveform.

In emerging fields or applications, it is not uncommon to develop near simultaneously the same experimental method by different groups. This process might generate a variety of names for the same method. For example, nanorheological $\mathrm{AFM}^{107}$ or dynamic scanning indentation ${ }^{108}$ describe similar FV configurations (see below). The use of chemically or biofunctionalized tips ${ }^{112,113}$ has led to several denominations, the oldest is chemical force microscopy ${ }^{114,115}$ and the newest are FD-based AFM or multiparametric AFM imaging. ${ }^{116}$ 
Chemical force microscopy. The operation of FV with chemically functionalized tips that terminate in well-defined functional groups has been used to measure adhesion forces between distinct chemical groups in aqueous solvents. ${ }^{114,115}$ The use of chemically functionalized tips enhanced the applications of FV, however, the chemical functionalization of the tip does not introduce any modification in the equation of motion, electronics, feedback or software of FV.

Multiparametric AFM imaging or FD-based AFM. The use of biofunctionalized tips in a FV experiment enables the detection of specific bimolecular recognition events. This feature enhances the applications in molecular and cell biology, ${ }^{110,116}$ however, in general, it should not be considered a new method.

The combination of FV and single-molecule force spectroscopy ${ }^{2,117}$ might lead to a new nanomechanical force spectroscopy method. Initial experimental results were promising ${ }^{118,119}$ but they involved a very small region of the sample mapped by FV. It is not straightforward to envision how the requirements of singlemolecule force spectroscopy, namely, the acquisition of multiple FDCs at different loading rates ${ }^{120}$ on the same position of the sample might be compatible with a fast acquisition of FV maps.

Ringing mode. This configuration is the most recent additions to FV. It is based on the use of the transient signal generated after breaking the tip-surface contact to generate compositional contrast maps. ${ }^{121}$ The transient signal provides additional parameters to characterize the tip-surface interaction. Those parameters might enhance material properties differences.

\subsection{The postulate of force spectroscopy}

Force-volume and AFM-based single-molecule force spectroscopy measurements are based on Hooke's law. Hooke's law establishes that the interaction force coincides with the cantilever deflection times its force constant (eqn (22)). Strictly speaking, Hooke's law is only valid for a static deflection, nonetheless, it was readily accepted by the AFM community ${ }^{4,18}$ without subjecting it to a rigorous demonstration.

The postulate of force spectroscopy was based on an empirical observation. In the 90's, force-distance curves were recorded at different modulation frequencies from high to low values. A FDC was considered acceptable if it did not depend on the frequency. This condition is easily met at low frequencies (see below). High-speed $\mathrm{AFM}^{6,122-124}$ has provided technologies to speed up FV measurements. However, performing a FV experiment at highspeeds (say 1 frame per $s$ ) requires a rigorous analysis of the transformation of a deflection into a force value. ${ }^{27,125}$

The equation of motion that describes force-volume (eqn (3)) shows the tip-surface $F_{\text {ts }}$ expressed in terms of the cantilever restoring force, an inertial term and a hydrodynamic force associated with the interaction of the cantilever with the fluid. To illustrate explicitly the dependence of the force on the modulation frequency, let's introduce a normalized time variable $\tau=\omega t$

$$
F_{\mathrm{ts}}(d(\tau), \dot{d}(\tau))=k z+\frac{k}{Q \varpi} \frac{\mathrm{d} z}{\mathrm{~d} \tau}+\frac{k}{\varpi^{2}} \frac{\mathrm{d}^{2} z}{\mathrm{~d} \tau^{2}}
$$

with $\varpi=\omega_{0} / \omega_{\mathrm{m}}$.
The identification of the tip-surface force with the cantilever restoring force requires to neglect the inertial and the hydrodynamic terms. This approximation is valid for $\varpi \gg 1$, this is, when the cantilever's fundamental frequency is much higher than the modulation frequency. In practice, to determine the Young's modulus with a relative error below $10 \%$ requires to use a frequency ratio above $50 .^{27}$ Therefore, to perform forcevolume measurements at high-speed rates in liquid (1 frame per $\mathrm{s}$ for $10^{4}$ pixels) might require the use of a cantilever with a fundamental frequency (in liquid) of about $1 \mathrm{MHz}$.

\subsection{Nanoscale rheology}

Nanoscale rheology describes the scientific activity and the FV configurations devoted to determine viscoelastic properties from FDCs. The goal is to assign one or several viscoelastic parameters to each pixel of the image. The deduction of viscoelastic parameters from a FDC demands significant theoretical and modelization efforts. ${ }^{49,93-96,126-135}$ Typical viscoelastic parameters measured in nanoscale rheology are loss and storage moduli, viscosity coefficient or loss tangent. In addition, a nanoscale rheology experiment might involve some changes in the experimental FV set-up.

The theory that relates FDCs observables to viscoelastic parameters depends on the type of waveform used to generate the tip-sample displacement (triangular versus sinusoidal). It might also depend on the existence of adhesion forces. For those reasons nanoscale rheology methods are divided in three sub-groups.

6.4.1 Nanoscale rheology with sinusoidal waveforms. The first attempts to measure viscoelastic parameters of living cells by AFM-based methods used very large spherical tips (polystyrene or silica beads) and applied either oscillatory ${ }^{90,136}$ or monotonic loads. ${ }^{137-139}$

An experiment performed by applying an oscillatory force might be decomposed in two steps. ${ }^{140}$ First, there is a pre-load step. The tip is indented on the cell until a pre-set force is reached. In a second step, a sinusoidal force is applied to the tip. The amplitude of the oscillation is smaller than the indentation depth reached in the first step, so the whole oscillation is performed with the tip indented on the material. Those experiments enabled to determine the storage and loss moduli of a cell as a function of the amplitudes of the force and the indentation. ${ }^{90}$ The use of large spherical tips prevented high-spatial resolution mapping.

Nanorheology experiments combine the above methods to measure FDCs with the use of sharp tips. In particular, the experiments performed without breaking the tip-sample contact during a FDC cycle are described by the linear viscoelastic theory developed for conventional nanoindentation experiments by Herbert, Pharr and Oliver ${ }^{141}$

For an axisymmetric tip, the effective storage and loss modulus are obtained by ${ }^{141,142}$

$$
E_{\mathrm{eff}}{ }^{\prime}=\frac{1}{2}\left(\frac{\pi}{S}\right)^{1 / 2} \frac{F_{0}}{A_{\mathrm{m}}} \cos \phi
$$




$$
E_{\mathrm{eff}}{ }^{\prime \prime}=\frac{1}{2}\left(\frac{\pi}{S}\right)^{1 / 2} \frac{F_{0}}{A_{\mathrm{m}}} \sin \phi
$$

where $F_{0}$ and $A_{\mathrm{m}}$ are the amplitudes, respectively, of the force and tip's displacement; $S$ is the projected contact area and $\rho$ is the phase shift between the force and the displacement.

6.4.2 Nanoscale rheology with triangular waveforms. The understanding of the nanomechanical response of a cell and the processing of FDCs are facilitated by using analytical theories. The combination of force-distance curves obtained with triangular waveforms and Kelvin-Voigt or power-law rheology models enables analytical theories. Those theories were applied to transform the FDCs into viscoelastic parameters $^{52,95}$ and FV maps. ${ }^{66}$ A short description of those approaches is provided in Section 4.2.

Other approaches. Schäeffer and co-workers performed nanoscale rheology experiments by using triangular waveforms with the addition of some loading and dwell steps. ${ }^{130,143}$ A FDC was obtained by approaching the tip towards the sample surface at a constant velocity. Upon an initial contact between the tip and the sample surface, the loading force increased until a pre-defined clamp-force was reached. This pre-defined force was kept constant during a dwell time before retracting the tip from the sample.

\subsection{Force-volume applications}

Force-volume is widely applied to characterize soft materials. Some recent results (2014-2020) are introduced to illustrate the capabilities, achievements and potential of the different FV configurations.

Materials science. Multiparametric mechanical property maps of polymer films, ${ }^{144}$ polymer blends ${ }^{145,146}$ and block co-polymer thin films ${ }^{147}$ are readily obtained. The interfacial region between a rubber matrix and silica nanoparticles was studied by using both nanoscale rheology ${ }^{107,148}$ and standard FV. ${ }^{149}$ These samples might be used as models of the internal structure of low rolling resistance tires. Novel polymer-based thin films are needed in packaging and environmentally friendly architectural outdoor coatings. FV characterizes the nanomechanical changes in the different elaboration steps of those coatings. ${ }^{150}$ Spatially-resolved elastic modulus maps have explained the morphology and composition of multicomponent polymer systems made of dissimilar polymers. ${ }^{151,152}$ An application at the interface in polymer science and biomedical devices involved the study of the swelling and degradation of biodegradable scaffolds. ${ }^{153}$ Chemical and nanomechanical mapping of polymers was achieved by combining FV and with broadband infrared spectra. ${ }^{154}$

Molecular biology. FV has found many applications in molecular biology. It has been applied to characterize the stiffness of DNA origami, ${ }^{155}$ protein complexes, ${ }^{156}$ protein- coated lipid nanotubes ${ }^{157}$ or liposomes. ${ }^{158}$ FV maps have recorded the changes in the mechanical properties of a virus. This has led to a more comprehensive characterization of the steps in virus maturation. ${ }^{159,160}$ Spatially-resolved maps of the adhesion force between a virus attached at the end of a tip and the plasma membrane has revealed the first steps of the binding of a virus to a mammalian cell. ${ }^{110}$

Cell biology and mechanobiology. One of the first FV measurements was performed on blood cells. ${ }^{14}$ Since then, FV has been applied to characterize different aspects of the structure and interactions of living cells. ${ }^{161-163}$ Some studies aimed to find a mechanical signature of disease at the cell level by comparing the mechanical properties of healthy and pathological cells. ${ }^{63,164-166}$ Elastic maps of collagen fibrils extracted from overloaded tendons were used to explain strain injuries. ${ }^{167}$ FV has shed light on the interactions driving adhesion and biofilm formation by microbial pathogens ${ }^{168}$ and on the surface properties of bacterial pathogens required to avoid host mediated immunity. ${ }^{169}$ Subsurface images of living cells have been generated by processing force-distance curves at different indentation depths. ${ }^{67,170,171}$ It has to be noted the considerable theoretical effort devoted to transform FDCs into viscoelastic properties. The overall result of this activity is poised to have an impact in mechanobiology. ${ }^{49,52,67,92-95,126-135}$

\section{Nanomechanical maps obtained from force-distance curves: on-resonance methods}

Force-distance curves are not directly measured in amplitude modulation AFM because the observables are averages over a period. Therefore, the observables (static deflection, amplitude and phase shift) are determined as a function of an average distance instead of the instantaneous tip-sample distance. To overcome this limitation several theoretical and experimental methods have been proposed. ${ }^{29-31,172-182}$ Those methods are classified in two groups, time-resolved ${ }^{29,30,172-176}$ and force reconstruction methods. ${ }^{177-182}$

To understand how a dynamic AFM method transforms the time dependencies of the observables into force-distance curves is pertinent to distinguish between flexural and torsional harmonics..$^{20,30,31,183}$ An harmonic oscillation vibrates at a frequency that is a multiple of the excitation frequency. In most AFM experiments, the deflection is measured in the plane perpendicular to the sample surface that contains the main axis of the cantilever. This is the flexural deflection (Fig. 7a). The harmonics of this motion are called flexural components. However, if the driving force has both vertical and lateral components, the deflection might have torsional components.

\subsection{Time-resolved force curves}

These methods provide the force in the time domain, that is, a force-time curve. A force-distance curve is directly obtained from the time-resolved force and the instantaneous tip's position. Three different approaches were developed to determine the instantaneous tip-sample force. One relies on the use of high-bandwidth active cantilevers, ${ }^{172-174}$ another on measuring the tip's acceleration ${ }^{176}$ and the other on detecting and processing the torsional harmonics. ${ }^{30,184}$ 
a
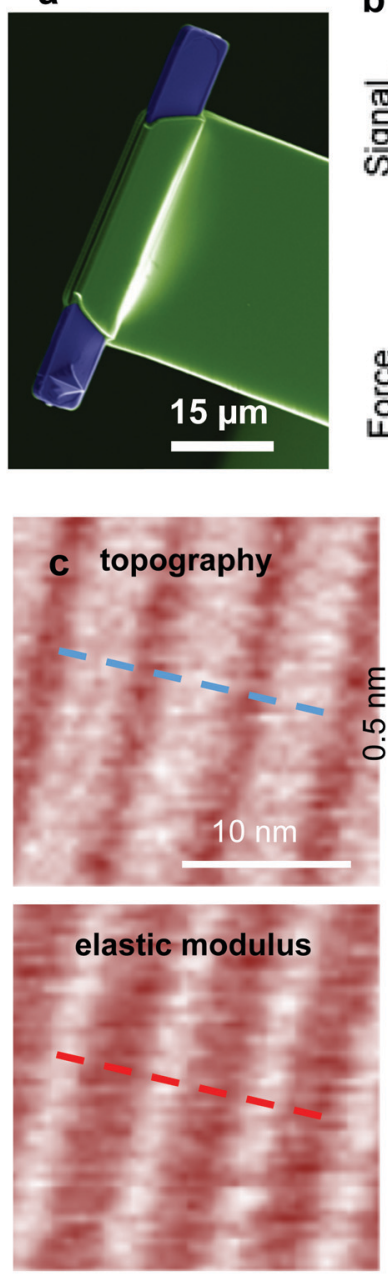

b



time

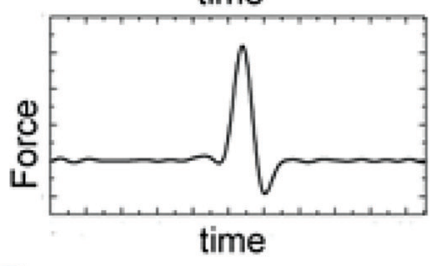

d
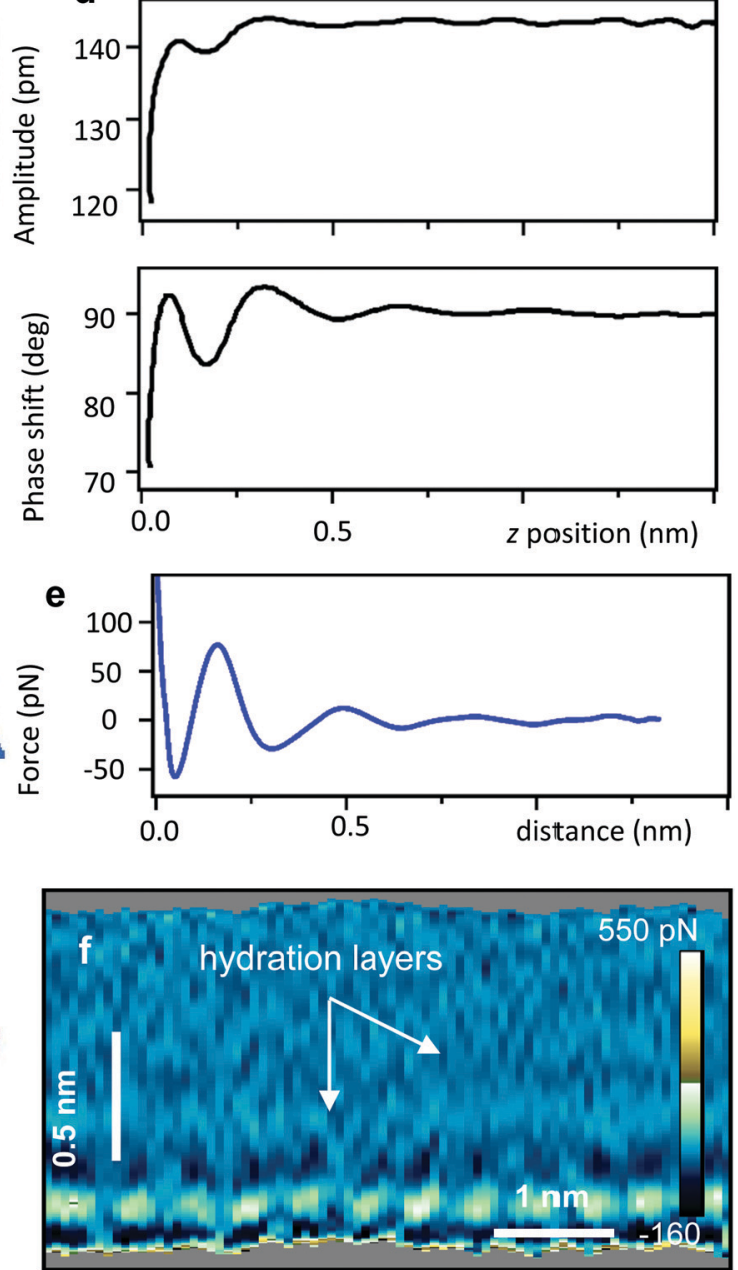

Fig. 7 (a) Torsional harmonic cantilever (scanning electron microscope image). Reprinted with permission from ref. 199. Copyright 2011 Springer Nature. (b) Flexural and torsional deflection signals. The force is obtained by integrating the torsional harmonic components. Reprinted with permission from ref. 30. Copyright 2007 Springer Nature. (c) Torsional harmonics nanomechanical map of amyloid nanostripped structures (Aß33-42). The height and elastic modulus map along the dashed lines are shown. Reprinted with permission from ref. 198. Copyright 2016 John Wiley and Sons. (d) Amplitude and phase shift-distance curves obtained by 3D-AFM on a mica-water interface. (e) Force-distance curve obtained from d. (f) 2D force panel generated by recording FDCs along the $x$-axis. The 2D force map shows the formation of hydration layers on a mica-water interface. ${ }^{205}$

High-bandwidth interdigitated cantilevers ${ }^{172-175}$ provide the instantaneous value of the force (force-time curve) on a point of the surface while generating a topographic image. These cantilevers have been applied to map several mechanical properties of living cells. ${ }^{185}$ Fabrication costs represents a major bottleneck for a widespread use of active cantilevers for nanomechanical mapping.

Scanning probe acceleration microscopy (SPAM) ${ }^{176}$ determines the force from the tip's acceleration. The acceleration of the cantilever is obtained by taking the second derivative of the cantilever deflection signal during an AM-AFM experiment in liquid. Once the acceleration is obtained, the second derivative of the cantilever trajectory is taken and multiplied by the effective mass of the cantilever to obtain the timeresolved force. This method has been applied to study lipid layers and lipid raft domains. ${ }^{186}$ The accuracy of SPAM relies heavily on the quality of the deflection signal. The noise in the deflection is significantly amplified by taking the second derivative.

7.1.1 Time-resolved forces by processing flexural harmonics. The capability to measure time-varying forces while taking a topographic image has been exploited to develop a timeresolved nanomechanical mapping method..$^{29,30,184}$ The force in the time domain is obtained by taking the inverse Fourier transform of the Fourier-transformed tip's trajectory divided by its transfer function. The non-linear character of the tip-surface force introduces a number of higher harmonic components in the cantilever's deflection (oscillation). ${ }^{187}$ The method requires the determination of a relatively large number of harmonics to provide a faithful reconstruction of the force $(\sim 10-20)$. The first time-resolved force reconstruction method was based on the measurement of flexural harmonic components. ${ }^{29,188}$ In general, the signal-to-noise ratio of the higher harmonics decreases by increasing the order of the harmonic component. ${ }^{31}$ This effect is 
unavoidable in standard rectangular cantilevers. A straightforward approach to overcome this effect is to increase the force applied to the sample to several hundreds of $\mathrm{nN}$ (peak force values). The drawback is that those forces end up damaging the tip ${ }^{189}$ and/or the soft material, in particular biomolecules. ${ }^{190,191}$ An alternative approach based on combining off and on-resonance features was suggested. ${ }^{192}$

Cantilevers were designed to enhance the generation of higher harmonics in the flexural deflection by removing the mass on certain sections of the microcantilever ${ }^{193,194}$ or by modifying the cantilever shape. ${ }^{195}$ However, those cantilevers have yet to be used in combination with the Stark et al. force reconstruction method. ${ }^{29}$

\subsection{Torsional harmonics}

Sahin and colleagues overcame the above limitations by introducing torsional harmonic microcantilevers. ${ }^{30}$ In a torsional harmonic cantilever the tip is offset from the cantilever axis (T-shaped) (Fig. 7a). In these cantilevers the tip-sample forces generate a torque around its main axis. This torque enhances the presence of the large number of higher harmonics needed for an accurate calculation of the force-distance curve. In addition, the optical sensitivity of the torsional deflection is higher than the corresponding for a flexural mode.

Torsional harmonics combines an amplitude modulation AFM feedback in the flexural deflection to generate a topographic image and the measurement the time-varying force during the tip's oscillation from the torsional harmonics. ${ }^{21,30,184}$ The force-distance curves are readily obtained from a force-time curve and the oscillation of the microcantilever.

The flexural and torsional deflections are processed independently. The torsional deflection is decomposed in the harmonic components of the frequency of driving force. The driving force is tuned to the first flexural resonance. The relationship between $F_{\mathrm{ts}}$ and $z$ is given by ${ }^{30,196}$

$$
F_{\mathrm{ts}}(\omega)=T^{-1}(\omega) z(\omega)
$$

with

$$
T(\omega)=c \frac{\omega_{t}^{2} / k_{t}}{\omega_{t}^{2}-\omega^{2}+i \omega \omega_{t} / Q_{t}}
$$

$F_{\mathrm{ts}}(t)$ is obtained by performing the inverse Fourier transform of $F_{\text {ts }}(\omega) . T$ is the transfer function of the torsional signal; the subscript $t$ indicates the 1 st torsional resonance; $c$ is a factor proportional to the torsional optical sensitivity.

Fig. $7 \mathrm{~b}$ shows the deflection of the torsional and flexural signals during a period of the flexural mode. High-spatial resolution topography and Young's modulus maps of amyloid peptide fibers are shown in Fig. 7c.

7.2.1 Applications. Torsional harmonic cantilevers can be operated at very small forces $(\sim 20 \mathrm{pN})$. This feature has been exploited to generate maps of the Young's modulus and stiffness of membrane proteins, protein fibers and lipid bilayers. ${ }^{20,197,198}$ Topography and molecular recognition imaging has also been achieved with this method. ${ }^{199}$ Torsional harmonics has revealed the different types of cell-cell junctions in cable bacteria filaments. ${ }^{200} \mathrm{~A}$ recent result is the measurement of the intracellular tension of actin bundles, cell cortex and plasma membrane. ${ }^{11}$ This result represents the first direct measurement of intracelullar forces. ${ }^{201}$ Torsional harmonics in combination with a microarray surface was proposed for the detection of DNA molecules at concentrations below femtomolar level. ${ }^{202}$

Torsional harmonics has also been called dynamical nanomechanical mapping. ${ }^{21}$ The method was commercialized under the name HarmoniX. ${ }^{\mathrm{TM}}$ Cantilever fabrication costs and the complexity of the calibration process of the torsional force constant have prevented a wider spread use of this method.

\subsection{Force-distance curves from amplitude modulation AFM}

In amplitude modulation AFM the observables cannot be expressed in terms of the instantaneous tip-sample distance because they are averaged over one oscillation cycle. This fact prevents the direct determination of the tip-sample force while imaging the topography. Several methods were developed to overcome this limitation by recording and processing amplitude and phase shift of the tip's oscillation as a function of the average tip-sample distance. ${ }^{177-182}$ Fig. $7 d$ shows some experimental amplitude and phase-shift distance curves. The amplitude and phase shift-distance curves can be generated either by using the standard AM-AFM configuration ${ }^{182}$ or by implementing other modulation methods. ${ }^{180,181}$ The integration enables to reconstruct the force from amplitude and phase shift-distance curves. In general, these methods are less efficient than FV because they involve more processing steps so very few applications have been reported. ${ }^{182,203}$ However, this method has have found an application niche in the characterization of solid-liquid interfaces (Fig. 7e) where the robustness and sensitivity of AM-AFM is especially suited for 3D-AFM imaging. ${ }^{204,205}$

\subsection{Other dynamic AFM methods}

Topography and recognition imaging (TREC) $)^{206,207}$ provides molecular recognition contrast maps. TREC is a qualitative nanomechanical contrast method. It is based on the operation of an AM-AFM in liquid with biofunctionalized tips. This method combines the tip's functionalization to detect specific interactions with the high-spatial resolution and speed of AM-AFM. This method relies on an asymmetry of the tip's oscillation in liquid. The repulsive tip-surface forces modify more strongly the shape of the lower part of the oscillation than its top part. The opposite happens when a tip-tethered ligand binds to a molecule on the sample surface. The molecules and the tether are stretched during the upward movement of the tip which decreases the top value of the oscillation. The stretching involves the transfer of mechanical energy from the cantilever to the molecule. This loss in the mechanical energy of the cantilever produces a decrease in the top peak of the oscillation. This method provides high-spatial resolution images of specific interaction events ${ }^{208-210}$ but it is not a quantitative method. It does not give values of the parameters that characterize a molecular recognition event.

Strain-stress curves are usually measured to determine the mechanical response of macroscopic materials. ${ }^{87}$ The stress-strain 
curve of a single antibody pentamer was measured by combining amplitude and frequency modulation AFM methods. ${ }^{191}$ For very small compressive forces $(5-75 \mathrm{pN})$, the protein's central region showed that the strain and stress are proportional (elastic regime). This region had an average Young's modulus of 2.5 MPa.

\section{Parametric methods}

Parametric methods are defined by the following features (Fig. 8a): (1) the interaction force is parameterized in terms of mechanical properties; (2) the tip's equation of motion is solved with the interaction force; (iii) analytic expressions are deduced to relate the observables and the mechanical parameters of the tip-sample interaction.

\subsection{Bimodal AFM}

Bimodal AFM involves the simultaneous excitation and detection of two modes of the cantilever. ${ }^{20,32,211}$ The use of two modes allows the determinations of several material properties such as topography, deformation, dissipation, elastic modulus, viscosity coefficients or long-range interaction parameters in a single imaging step. ${ }^{86,211-216}$ Most bimodal experiments are performed by using the first two flexural modes ${ }^{20}$ of the cantilever, however, other eigenmodes either flexural ${ }^{217}$ or a combination of flexural and torsional modes have been used. $^{218-220}$

In bimodal AFM, the tip's motion is decomposed in terms of the components oscillating at the frequencies of the excited modes $^{214,221}$ (Fig. 8b). Each mode might contribute with up to three observables, the amplitude $A_{i}$, phase shift $\phi_{i}$ and resonant frequency shift $\Delta f_{\mathrm{i}}$. The values and roles of the excited modes are not symmetrical. The amplitude of the first mode $A_{1}$ is about 10 times larger than $A_{2}$. Therefore, the observables of the 1 st and 2 nd mode explore different ranges of the interaction force. $^{222,223}$

The type and number of observables used to run the feedback loops have generated several configurations. ${ }^{211}$ The first
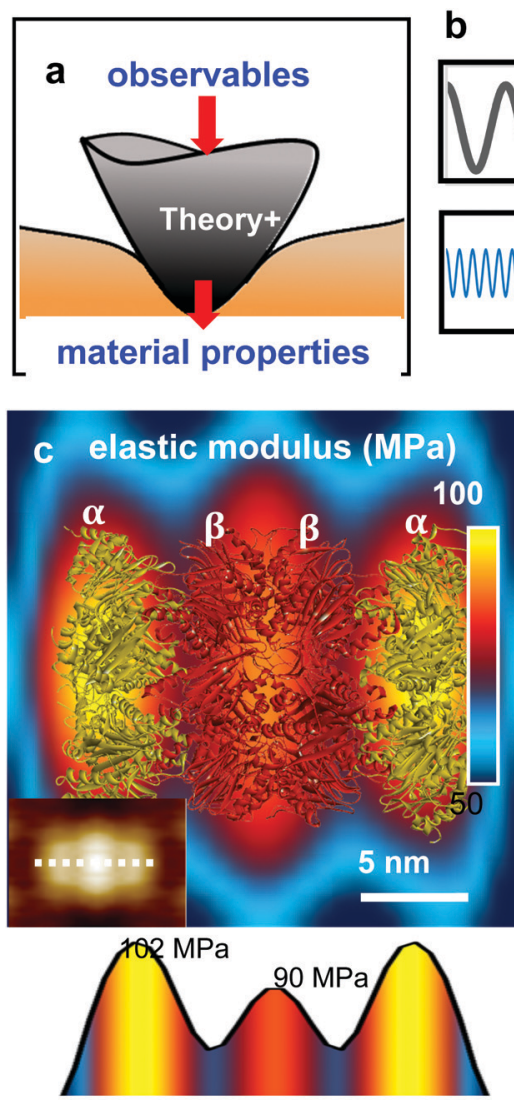

\section{b}

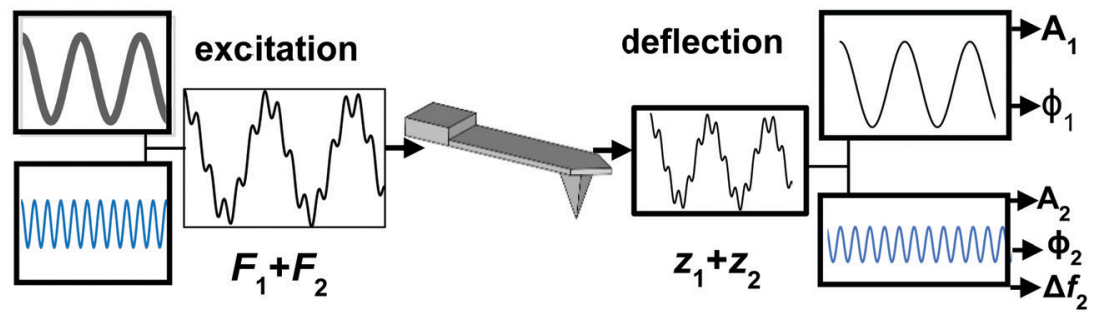

height reconstruction
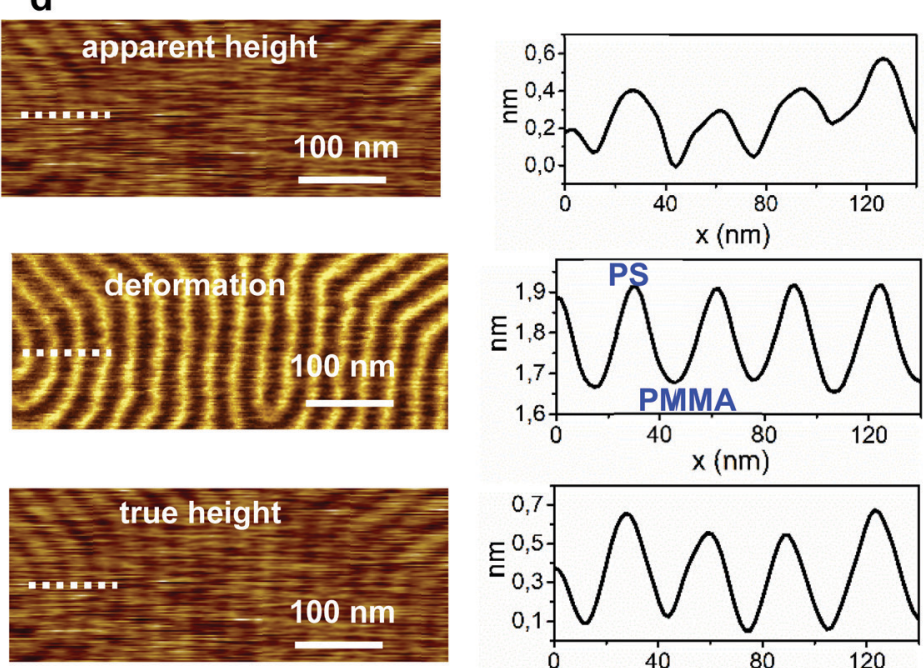

Fig. 8 Bimodal AFM. (a) General bimodal AFM scheme to transform observables into material properties. (b) Excitation and detection scheme. The cantilever is excited at its first two eigenmodes. Upon interaction with the sample, the components of the tip's response are processed. This step generates several observables. In bimodal AM-FM, the topography is obtained by keeping $A_{1}=$ constant while two feedback loops act on the parameters of the 2 nd mode (see ref. 24). (c) Nanomechanical map of a single protein (20S proteasome). The map shows a correlation between the elastic modulus and the gate $\alpha$ and catalytic $\beta$ subunits. The protein structure is overlaid. The panel at the bottom shows the elastic modulus cross-section along the dashed line marked on the topographic image (inset). Inset adapted with permission from ref. 24. Copyright 2018 SpringerNature. (d) Height reconstruction from the apparent height and deformation data. True height reconstruction. From top to bottom, apparent topography, deformation and true height of a PS-b-PMMA film. ${ }^{216}$ The PS domains have larger deformations (bright) than the stiffer PMMA domains (dark). The nanomechanical maps and images were generated simultaneously during imaging. 
bimodal AFM configuration had a single feedback loop acting on the amplitude of the first mode..$^{224,225}$ The latest configuration involves a feedback loop on $A_{1}$ for topography and a feedback on the frequency shift of the 2nd mode for sensitivity. ${ }^{214,215}$ The existence of several bimodal AFM configurations have motivated a significant activity in theory, simulations and instrumentation. ${ }^{226-238}$

In bimodal AFM the number of unknowns about the interaction force matches the number of equations. The availability of analytical expressions enables the simultaneous mapping of topography and mechanical property mapping. Numerical simulations have validated the accuracy of the bimodal AFM theory. ${ }^{214}$

8.1.1 Bimodal AM-FM. In this configuration, ${ }^{24}$ the amplitude of the first mode (AM) is used as a feedback signal for controlling the tip-sample distance. This feedback loop generates an apparent image of the topography of the sample. A second feedback loop acts on the deflection of the second mode by keeping constant the phase shift at $\phi_{2}=90^{\circ}$. At the same time, another loop acts on the driving force of the second mode to keep the oscillation amplitude of the second mode at a fixed value $A_{2}=$ constant. The latter loops share some features of frequency modulation AFM. ${ }^{38}$ This configuration combines the robustness of AM-AFM with the sensitivity and enhanced signal-to-noise ratio of a frequency modulation AFM.

The theory of bimodal AFM approximates the tip's deflection by ${ }^{24,214}$

$$
\begin{gathered}
z(t) \approx z_{0}+z_{1}(t)+z_{2}(t)=z_{0}+A_{1} \cos \left(2 \pi f_{1} t-\phi_{1}\right) \\
+A_{2} \cos \left(2 \pi f_{2} t-\pi / 2\right)
\end{gathered}
$$

The theory is based on the application of the virial and energy balance equations to the each of the excited methods. The explicit solution of those equations requires a tip-surface force model. Analytical solutions have been deduced for several elastic contact mechanics models ${ }^{24214,223}$ and for a linear viscoelastic model (3D Kelvin-Voigt). ${ }^{216}$

To illustrate how bimodal AM-FM generates a nanomechanical map (Fig. 8c), let's focus on the expression of the effective elastic modulus for a material described by Hertz model ${ }^{24}$

$$
\begin{aligned}
& E_{\mathrm{eff}}(x, y)=B\left(\frac{k_{2} \Delta f_{2}}{k_{1} f_{02}}\right)^{2} \\
& B=\frac{4 \sqrt{2}}{\sqrt{R}} k_{1} Q_{1} \frac{A_{1}^{3 / 2}}{A_{01}{ }^{2}-A_{1}^{2}}
\end{aligned}
$$

where $B$ is a conversion factor that depends on parameters of the cantilever-tip (radius, force constants, quality factor) and free amplitude $A_{01}$ the feedback parameters $A_{1}$. Those parameters are set and the beginning of the measurement and remain constant during the experiment. Therefore, the spatial dependence of the elastic modulus is given by measuring the local changes of the cosine of $\phi_{1}$. Expressions for the energy dissipated, virial, elastic modulus, loss tangent or viscosity coefficient are found elsewhere. ${ }^{24,216,223}$
8.1.2 True topography. The force exerted by the tip on a soft material produces its deformation. In fact, this observation is what enables nanomechanical property mapping. However, sample deformation, even in a linear elastic regime, might have some unwanted implications. The data coming directly from the topographic feedback represents the apparent topography of the surface $h_{\mathrm{a}}$. This is a well-known effect ${ }^{86,191,239-241}$ which is caused by a variety factors such as the existence of long-range electrostatic forces, ${ }^{240}$ the local tip-sample geometry, ${ }^{241}$ or the deformation either elastic ${ }^{191,239}$ or inelastic produced by the applied force. ${ }^{191}$ However, this effect is commonly ignored. It is assumed that the differences between true and apparent heights are negligible. This assumption might not be met during imaging of soft materials, in particular, biomolecules. In conventional AFM is not straightforward in a single step to determine the true topography because the same observable (deflection) carries the information on the deformation and the topography.

Bimodal AFM solves the above issue because the deformation induced by the force applied by the tip is directly determined from the observables. ${ }^{24}$ Then, the true topography can be obtained by applying the following equations,

$$
\begin{gathered}
h_{\text {true }}=h_{\mathrm{a}}(x, y)+I_{\max }(x, y) \\
I_{\max }=\frac{k_{1} f_{02}}{2 Q_{1} k_{2} \Delta f_{2}}\left(A_{02}{ }^{2}-A_{1}^{2}\right)^{1 / 2}
\end{gathered}
$$

where $I_{\max }$ is the maximum deformation of the sample in a period.

Fig. 8d shows the apparent height, the deformation and true height images of a poly(styrene-block-methyl methacrylate) (PS- $b$-PMMA) film. The diblock copolymer arranges in an ordered lamellar structure alternating PS and PMMA domains with a pitch of $30 \mathrm{~nm}$. By measuring the elastic modulus, bimodal AFM enables to associate the low elastic modulus (2.1 GPa) to the PS domains and the high elastic modulus $(2.6 \mathrm{GPa})$ to the PMMA domains. ${ }^{216}$ Under the application of a peak force of $12.5 \mathrm{nN}$, the softer PS regions show a total deformation of $1.9 \mathrm{~nm}$ while the deformation of the PMMA domains is of $1.65 \mathrm{~nm} .{ }^{216}$ Those values show that the deformation exerted by the tip on a soft material are significant.

8.1.3 Bimodal AFM applications. The following examples, taken from the 2014-2020 period, illustrate the capabilities, potential and achievements of bimodal AFM.

Materials science. Nanomechanical maps have characterized the performance of organic electrochemical transistors. Those maps showed that an increase of the stiffness of an organic electronic material decreases ion uptake which, in turns, decreases the performance the transistor. ${ }^{242}$ Local spin transitions in thin molecular films have been determined by following the temperature dependence of the Young's modulus from the low to the high spin states. ${ }^{243}$ In another application, it was reported that the surface elasticity of binary self-assembled monolayers increased with ligand ordering. ${ }^{244}$

The local elastic and inelastic properties of polymer films, polymer-based materials ${ }^{214,245}$ and flexible crystals ${ }^{246}$ have 
been extensively studied by bimodal AFM. In particular, the structure and elastic properties of semi crystalline polymers has been obtained on a molecular-scale level. ${ }^{215}$ The elastic properties $^{247}$ and wettability transparency ${ }^{248}$ of two-dimensional materials were also studied.

Molecular and cell biology. Bimodal AFM showed that the physical properties of lipid bilayers are modulated by the concentration of cholesterol. Regions with a low concentration of cholesterol molecules are predominantly elastic while regions with a high concentration have viscoelastic properties. ${ }^{249}$ High-spatial resolution nanomechanical maps characterized the adsorption of single alkali cations on lipid bilayers. ${ }^{250}$ It was reported that the formation of an ionic network reduces the elastic modulus of the lipid bilayer. Elastic property maps characterized the distribution of magnetic nanoparticles adsorbed and inside in liposomes. ${ }^{251}$

High-spatial resolution maps of the elastic properties of membrane proteins, ${ }^{214}$ single proteins, ${ }^{24}$ protein fibrils ${ }^{252,253}$ and $\mathrm{DNA}^{215,254}$ were generated with a lateral resolution in 1 to $5 \mathrm{~nm}$ range. Nanomechanical maps of other biological systems such as bone elements, ${ }^{255}$ virus-like particles ${ }^{256}$ and cells have been reported. ${ }^{257,258}$

Instrumentation. Bimodal AFM has inspired several detection schemes for probe microscopy methods..$^{259-261}$ It has also motivated the design of novel cantilevers. ${ }^{262-265}$

\subsection{Contact resonance and multi-harmonic methods}

Other experimental and signal processing parametric schemes were developed to map mechanical properties. ${ }^{266-268}$ Contact resonance AFM tracks the changes of the cantilever's resonant frequency induced by the changes of the mechanical properties of the tip-sample contact. ${ }^{266}$ Multi-harmonic AFM assumes a tip's oscillation dominated by just three harmonic components and analytical expressions to relate those components to local mechanical properties. ${ }^{267}$

8.2.1 Contact resonance AFM. Contact resonance AFM (CR-AFM) evolved from atomic force acoustic microscopy. ${ }^{33,266,269}$ In this method the sample is excited by ultrasound frequencies while the tip and sample remain in contact. The resonant frequency, the amplitude and the phase shift of the cantilevertip response are measured and compared to those of the free standing tip. Theoretical models were developed to relate the observables to the contact stiffness for an elastic contact ${ }^{270}$ or to the contact stiffness and damping coefficients for a viscoelastic contact. ${ }^{271,272}$ CR-AFM has two main configurations depending whether the ultrasound excitation is applied to the base of the cantilever269,273 or to the sample support. ${ }^{266}$

The theory of CR-AFM establishes that the stiffness and damping parameters of a contact described by the Kelvin-Voigt model are related to the loss and storage modulus by ${ }^{271}$

$$
E_{\mathrm{eff}}^{*}(x, y)=E_{\mathrm{eff}}{ }^{\prime}+i E_{\mathrm{eff}}{ }^{\prime \prime}=\frac{k_{\mathrm{ts}}}{2 r_{\mathrm{c}}}+i \frac{\pi \omega c_{\mathrm{ts}}}{r_{\mathrm{c}}}
$$

where $E^{*}, E^{\prime}$ and $E^{\prime \prime}$ are, respectively, the complex, storage and loss moduli; $k_{\mathrm{ts}}$ and $c_{\mathrm{ts}}$ are, respectively, the contact stiffness and contact damping parameters. The above expression was deduced by using the projected contact area given by Hertz model.

The application of eqn (33) involves several steps. One of them requires fitting the experimental frequency response with a theoretical model. Accurate determination of the contact radius $r_{\mathrm{c}}$ might be problematic which led to use of reference samples with well-known mechanical properties. ${ }^{23,271}$

The spatial resolution of a nanomechanical map obtained by CR-AFM is rather modest if compared to the spatial resolutions achieved in either FV or bimodal AFM. In general, a contact resonance AFM experiment involves the application of relatively large forces, say of a few hundreds of $\mathrm{nN}^{274}$ Large forces might damage the surface and worsen the spatial resolution. For those reasons, the application of CR-AFM to soft materials have been limited to a few polymers with relatively high elastic modulus $(\sim 1 \mathrm{GPa}) .^{23,273}$

Contact resonance AFM drew some attention for imaging in a non-invasive way subsurface features in polymer blends, plant cells or bacteria. ${ }^{275-278}$ However, the combination of a modest spatial resolution and the complexity of the models needed to interpret the data ${ }^{279-281}$ has slowed down progress.

8.2.2 Multi-harmonic AFM. Multi-harmonic AFM ${ }^{267,282,283}$ is based on processing a few multi-harmonic components of the deflection signal recorded during amplitude modulation AFM imaging. It assumes that the steady state motion of the tip is periodic and it involves 3 or at most 4 harmonics of the drive frequency, the 0th, 1st, 2nd and perhaps the 3rd. It considers also that a periodic oscillation implies a periodic tip-sample force. The above considerations enabled to express the interaction force as a Taylor series of those components. By applying the energy balance and virial theorem some analytical expressions were derived that link the Fourier components of the force to the multi-harmonic observables (amplitudes and phase shifts). A contact mechanics model is needed to parameterized the force into material parameters. ${ }^{282}$ Multi-harmonic AFM requires to evaluate some integral equations to link the observables into mechanical parameters involves.

Multi-harmonic AFM was applied to map of viscoelastic parameters of cells. ${ }^{28,284,285}$ The softness of a cell implies that the resonance curve of the cantilever is weakly distorted by the interaction with the cell. This observation is consistent with assuming an oscillation with just three harmonic components. The method has been extended to measure the mechanical properties of stiffer samples such as viruses. ${ }^{286}$

\section{AFM phase-imaging}

AFM phase-imaging has significantly contributed to the establishment of force microscopy as a key nanoscale characterization method in materials science. ${ }^{5}$ In the mid-90s, amplitude modulation AFM phase contrast images provided the first high-spatial resolution maps of polymer surfaces in their native conditions and state ${ }^{16,17,287-291}$ Some of the images of block-copolymer interfaces could be considered iconic. ${ }^{292,293}$ Nowadays, AFM phase-imaging is routinely used in the characterization of heterogeneous soft materials at the nanoscale. 
a

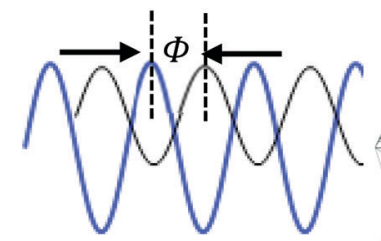

h
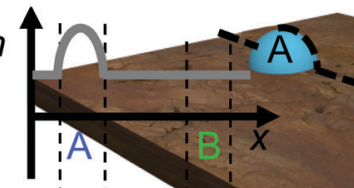
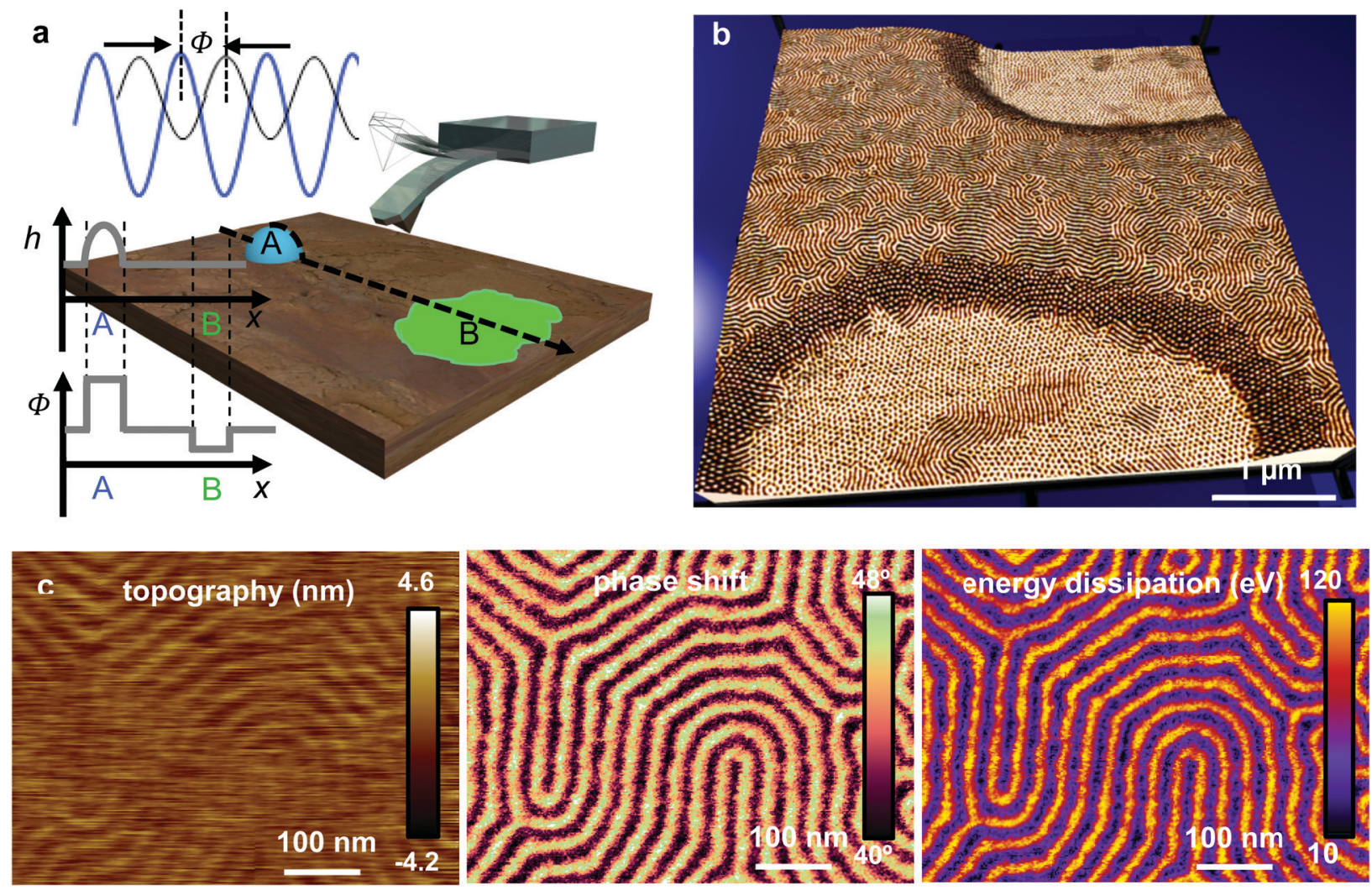

Fig. 9 AFM phase-imaging. (a) Scheme of phase shift contrast. The lag of tip's response with respect to the driving force is the phase shift $\phi$. Phase shift contrast might happen in heterogenous but otherwise flat samples (b) Reconstructed image of a polystyrene-block-polybutadiene-block-polystyrene (SBS) film. The image was formed by combining topographic and phase shift images. The image shows the formation of terraces. The structure of the domains change with the film thickness. In the lower terrace (film thickness of $32 \mathrm{~nm}$ ) the cylinders are oriented perpendicular to the support. In the higher terrace $(57 \mathrm{~nm})$ the domains are parallel to the underlying support. Reprinted with permission from ref. 293. Copyright 2004 AlP Publishing. (c) Topography, phase shift and energy dissipation images of PS-b-PMMA film. ${ }^{216}$

AFM phase-imaging is an element of amplitude modulation (tapping mode) AFM. ${ }^{31,37}$ An AFM phase contrast image is obtained by recording the phase shift on each point of the surface $\phi(x, y)$ while imaging the surface topography (Fig. 9a). Thus, topography and phase contrast images are acquired simultaneously (Fig. 9b and c).

The amplitude of the tip's oscillation is controlled by the convolution of the interaction force and the tip's position and by the amount of energy that the tip dissipates on the sample in one oscillation. ${ }^{294}$ On the other hand, the sine of the phase shift is directly proportional to energy dissipated by the tip on the sample. ${ }^{295-297}$ Thus, in AM-AFM the amplitude and the phase shift are controlled by different physical factors. This property enables AFM phase-imaging to resolve features that might be hidden in the topographic signal. The key role of energy dissipation in explaining the contrast in AFM phaseimaging might be counter-intuitive. It created some conflicting interpretations that lasted from the early days ${ }^{298-305}$ to the development of theory for imaging in liquid..$^{306-308}$

\subsection{Theory of AFM phase-imaging}

Let's consider a cantilever-tip system that is driven by a sinusoidal force near a surface. In general, the phase shift between the tip's oscillation and excitation force depends on both the elastic and the inelastic components of the interaction force. ${ }^{294,308}$ This is a general property of oscillating systems. However, in AM-AFM the feedback control imposes the condition of keeping $A=$ constant while imaging. This condition implies that, for moderate to high quality factor cantilevers $(Q \geq 10)$, the phase shift contrast depends exclusively on energy-dissipative processes. ${ }^{295-297,309,310}$ Moderate to high $Q$-factors are commonly found in experiments performed in air or vacuum.

The general solution of eqn (1) gives the deflection in terms of the components of harmonics $(n \omega)$ of the frequency of the driving force $F_{0} \cos \omega t$

$$
z=z_{0}+\sum_{n=1}^{\infty} A_{n} \cos \left(n \omega t-\phi_{n}\right)
$$

where $z_{0}$ is the mean deflection of the cantilever and $A_{n}$ is the amplitude of the $n$th harmonic.

Energy balance applied to a complete period of the tip's oscillation imply that the work performed by the driving force must coincide with the sum of the total energy dissipated by the tip-surface forces $E_{\mathrm{dis}}$ and the energy dissipated in the medium 
(air or liquid) $E_{\text {med }}$. Then, it was deduced ${ }^{306,308}$

$$
\sin \phi_{1}=\sum_{n=1}^{\infty} \frac{n^{2} A_{n}^{2} \omega}{A_{0} A_{1} \omega_{0}}\left(1+\frac{E_{\mathrm{dis}}}{E_{\mathrm{med}}}\right)
$$

The above expression establishes that the phase shift depends on two factors, the generation of higher harmonic components $A_{n}$ and energy dissipation. The dependence with the energy dissipated by the tip in the sample is explicit while the dependence on the elastic interaction is implicit in the generation of $A_{n}$. Consequently, phase shift variations will depend on both conservative and non-conservative interactions. However, for moderate-to-high $Q$ cantilevers $(\geq 10)$ the amplitudes of multi-harmonic components are negligible with respect to the amplitude of the fundamental harmonic $A_{1}\left(\left(A_{n} / A_{1}\right)^{2} \ll 1\right.$ with $n \geq 2) .{ }^{31}$ This leads to

$$
\sin \phi_{1}=\frac{A_{1} \omega}{A_{0} \omega_{0}}\left(1+\frac{E_{\mathrm{dis}}}{E_{\mathrm{med}}}\right)
$$

where $A_{1}, A_{0}, E_{\text {med }}$ are set at the beginning of the experiment, consequently

$$
\phi(x, y)=\sin ^{-1}\left[c_{1}+c_{2} E_{\mathrm{dis}}(x, y)\right]
$$

$c_{1}$ and $c_{2}$ remain constant during the imaging. Therefore, the spatial changes of $\phi(x, y)$ are directly related to change in $E_{\text {dis }}(x, y)$. By reordering terms in eqn (36),

$$
E_{\mathrm{dis}}(x, y)=E_{\mathrm{med}}\left(\frac{A_{0} \omega_{0} \sin \varphi(x, y)}{A_{1} \omega_{1}}-1\right)
$$

The validity of eqn (36) and (38) was confirmed experimentally ${ }^{78,296}$ and by using numerical simulations. ${ }^{39,308,312}$ Eqn (38) is extensively applied to measure energy dissipation processes at the nanoscale. ${ }^{309-315}$ This equation was used to determine the $E_{\text {dis }}$ as a function of the tip-sample separation (energy-dissipation curve). Those curves were applied to identify dissipation processes at the nanoscale. ${ }^{39}$

Fig. 9c shows the apparent height, phase shift and energy dissipation maps of a PS- $b$-PMMA film. Both the phase shift and the energy dissipation maps show contrast between the PS and the PMMA domains. However, the images by themselves do not allow to associate a given region with either a PS or a PMMA domain. This is a consequence of the semi-quantitative character of AFM phase-imaging (see Section 10.1).

\subsection{The paradox of AFM phase-imaging}

Does the phase contrast measured between two points $\Delta \phi=$ $\phi\left(x_{1}, y_{1}\right)-\phi\left(x_{2}, y_{2}\right)$ involve necessarily local changes in the nonconservative component of the force $\mathrm{F}_{\text {dis }}(x, y, z)$ ? In general, the answer is no. Once there is a dissipative interaction, local changes in the elastic parameters of the sample could give rise to local phase shift changes.

The application of the virial theorem ${ }^{294}$ to the tip's motion enabled to determine a expression for $\cos \phi(x, y)$. That expression together with eqn (37) enabled to calculate the dissipated and stored energy of the tip as a function, respectively, of sin $\phi(x, y)$ and $\cos \phi(x, y)$. Proksch combined those expressions to map loss tangent variations in polymeric films. ${ }^{316,317}$

\subsection{Applications of AFM phase-imaging}

AFM phase contrast images have contributed to solve many problems in materials science. Let's introduce some examples from the 2014-2020 period. The phase shift signal was used to generate subsurface images of polymeric materials. ${ }^{318,319}$ The hydration of biopolymers under different conditions was followed by recording phase shift-distance curves. ${ }^{320,321}$ Several viscoelastic processes on polymer blends and polymer nanocomposites have been studied by AFM phase contrast images. $^{322,323}$ The sensitivity of the phase shift signal has enabled to detect interfacial fluctuations on PS- $b$-PMMA, ${ }^{324}$ to perform in situ studies of crystallization of polymers as a function of the temperature ${ }^{325}$ or to image in realtime selfassembly processes. ${ }^{326}$ The compatibility of AFM phaseimaging and high-speed AFM was demonstrated by imaging membrane vesicles isolated from different bacterial strains at $2 \mathrm{~s}$ per frame $(200 \times 200$ pixels $) .{ }^{327}$

\section{Open issues and future developments}

Does a nanomechanical map has less spatial resolution than topography imaging? To what extent continuous mechanics models are valid to describe the measurements? Those are examples of some fundamental questions in nanomechanical mapping. Other issues are more technical in nature. Recent technology and scientific advances such as high-speed operation, big data analysis and machine learning are about to influence the field. Let's examine in detail some of the issues that are likely to shape the future of nanomechanical force spectroscopy.

\subsection{Quantitative versus compositional contrast methods}

The main goal of the field is to develop fast and high-spatial resolution methods that provide values of a mechanical property that either has a well-defined macroscopic counter-parts or measures an intrinsic nanoscale property. Examples of the former are the storage and loss modulus. An example of the latter might be the determination of the energy of an intermolecular bond.

Force-volume, bimodal AFM, contact resonance or torsional harmonics provide maps of mechanical properties. Those methods are quantitative. Topography and recognition imaging and chemical force microscopy rely on the detection and/or measurement of adhesion forces. Adhesion forces might not have a direct relationship with a mechanical property of the material because they depend on the contact area. Those methods are qualitative. Qualitative methods might be useful to generate high-resolution compositional contrast images or to detect the position of a receptor molecule. AFM phaseimaging is a singular case. It measures the energy dissipated by the tip on the sample. This is not a mechanical property; however, phase shift maps might be transformed into spatiallyresolved measurements of loss tangent values. 


\subsection{Spatial resolution}

Does nanomechanical mapping of soft materials imply less spatial resolution? This question might not have a simple answer. First, the spatial resolution will depend on the type of mechanical property. Second, the methods discussed in this review are not equivalent in terms of spatial resolution and sensitivity.

An estimation of the spatial resolution $l_{\mathrm{r}}$ for a tip of radius $R$ when long-range attractive forces are negligible with respect to the value of the repulsive force is given by ${ }^{31}$

$$
l_{\mathrm{r}}=2\left(\frac{3 R F_{\mathrm{ts}}}{4 E_{\mathrm{eff}}}\right)^{1 / 3}
$$

the lateral resolution depends on tip's radius, applied force and elastic modulus. According to eqn (39) a nanomechanical map should have the same spatial resolution as the topographic image. An applied force involves a certain signal-to-noise ratio in the deflection signal. How the error propagates into a given image/map depends on the processing path associated with that image/map. In general, a nanomechanical map processes more observables than a topographic image. Therefore, a higher error, this is, less spatial resolution might be expected.
Let's illustrate the above consideration by comparing highspatial resolution maps of membrane proteins.

The topography of native purple membranes was studied by Muller and co-workers. ${ }^{328}$ This surface might be considered a calibration standard for very high-resolution imaging of biomolecules in aqueous solutions. Fig. 10a shows a scheme of the membrane structure. The purple membrane consists of $75 \%$ bacteriorhodopsin (BR) and 25\% lipids (w/w). BR forms a $2 \mathrm{D}$ trigonal crystal $(a=b=6.2 \pm 0.2 \mathrm{~nm})$. The position of the transmembrane $\alpha$-helices are marked.

Fig. 10b shows a state-of-the-art image of the extracellular BR surface obtained by AFM (contact mode). ${ }^{328}$ A single defect of the size of a BR trimer is marked. Fig. 10c and d show bimodal AFM maps of the EC. ${ }^{214}$ The bimodal image (topography) shows the BR trimers in an hexagonal arrangement. The image reveals the trimer's lattice and single defects (marked). The contrast might not be as good as in Fig. 10b but there is no loss in spatial resolution. The elastic modulus map (raw data) shows a regular pattern with a $6.2 \mathrm{~nm}$ periodicity but the hexagonal structure is not readily visible (Fig. 10d). Cross-correlation and averaging methods were applied to reveal the trigonal structure (inset). The Young's modulus across the BR trimers changes between 30 to $40 \mathrm{MPa}$. Fig. 10e and f shows some FV topographic and elastic modulus maps of the EC side of PM. ${ }^{111}$
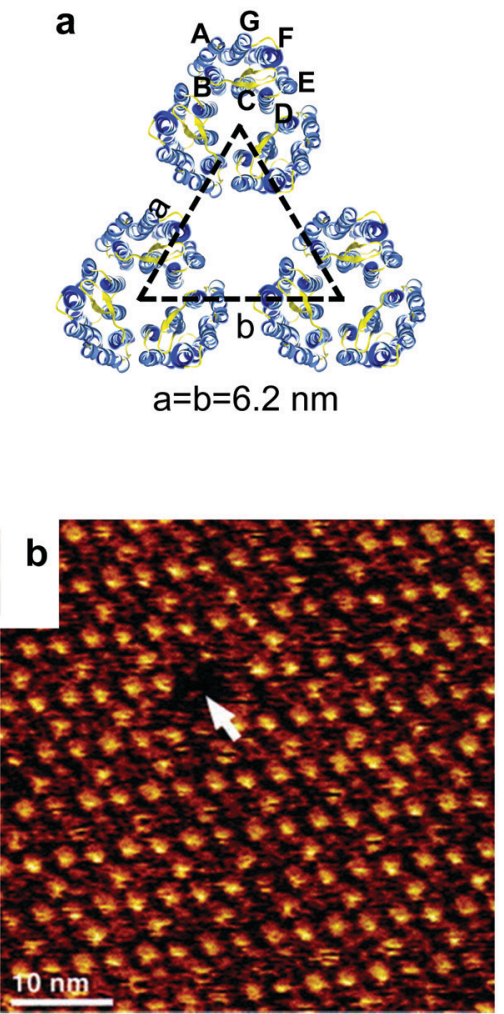
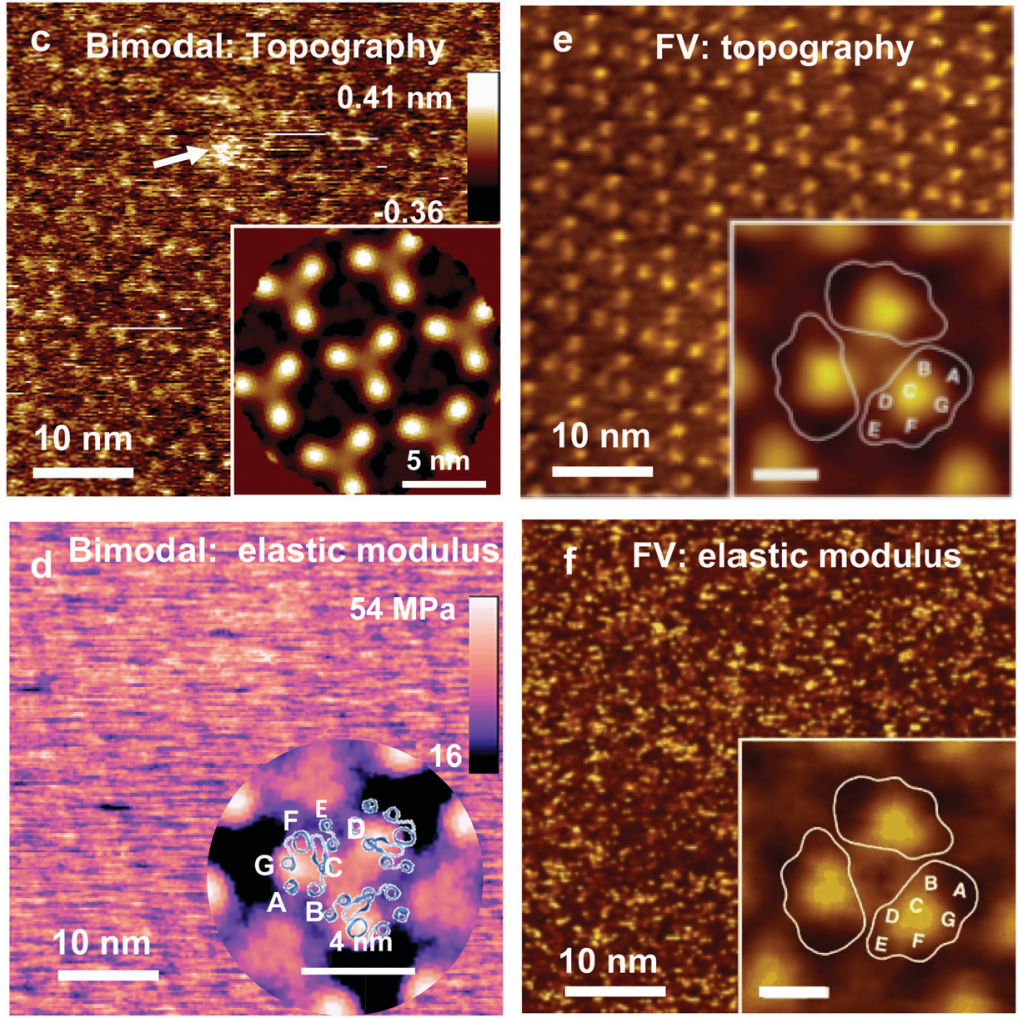

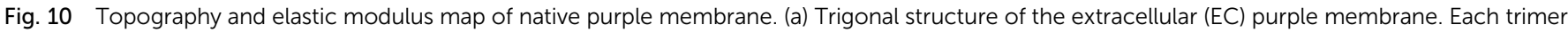

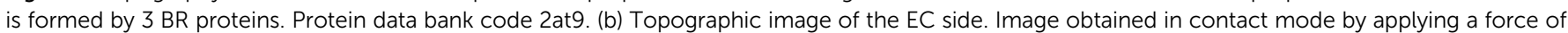

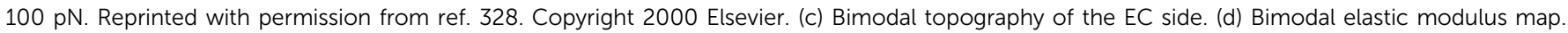




ref. 111. Copyright 2011 John Wiley and Sons. The insets in (c-f) and show the 3-fold symmetrized averages. 
The topographic image shows the hexagonal arrangement of the BR trimer (Fig. 10e). The elastic modulus map is very noisy without providing any hint on the BR structure (Fig. 10f). Extensive averaging methods were applied to reveal the periodicity (inset).

This example shows that the spatial resolution in the topography is not affected by nanomechanical mapping (Fig. 10b, c and e) while the spatial resolution of the elastic modulus maps (raw data) is diminished (Fig. 10d and f). Nonetheless, the above conclusion is not general. The spatial resolutions topographic and elastic modulus maps of some proteins $^{24}$ and block-copolymers ${ }^{216}$ were for all purposes identical.

\subsection{Quantitative accuracy}

The accuracy of a nanomechanical measurement is controlled by three independent factors: the model or theory that describes the mechanical properties of the sample, the theory that transform observables into properties and the calibration of the instrument.

10.3.1 Theories and models. The determination of material property values requires two theoretical steps. First, a model that relates forces, deformations and properties. Second, a theory that transforms the observables into quantitative values of material properties. Either contact mechanics theories or mechanical models, this, models based on mechanical analogues are used to solve the constitutive equations. Therefore, the accuracy of a measurement will be limited by the suitability of the model or theory used to describe the mechanical properties.

The second factor was rigorously addressed by the theories of bimodal $\mathrm{AFM}^{214}$ and force-volume ${ }^{27}$ configurations. Let's assume that for a given material there is a contact mechanics model that describes its mechanical properties. What is the relative error incurred, for example, in the determination of the Young's modulus? A numerical simulator designed to describe bimodal AM-FM operation ${ }^{214}$ showed that the Young's modulus can be determined with a relative error below 5\% over a 5 orders of magnitude range ( $1 \mathrm{MPa}$ to $100 \mathrm{GPa})$. Similar results were obtained for the determination of the viscosity coefficient and the retardation times of the $3 \mathrm{D}$ Kelvin-Voigt model. $^{216}$

Numerical simulations of the equation of motion ${ }^{27}$ and FEM $^{44,65,95}$ simulations were used to establish the operating conditions in FV to map elastic and viscoelastic properties with accuracy. Theoretical expressions and numerical simulations defined the frequency ratios required to determine the elastic modulus of a material with an error below $5 \%$ by $\mathrm{FV}^{27}$ The validity of the bottom-effect correction expressions were tested by comparing the force-distance curves deduced from the bottom effect theory with the force-distance curves obtained from finite element method (FEM) simulations. ${ }^{44,65}$ Those comparisons showed the conditions to determine elastic and viscoelastic parameters with a relative error below $10 \%$. The accuracy of AFM phase-imaging to determine the energy dissipation and loss tangent values was discussed in Section 9.1.

10.3.2 Force constant and tip's radius calibration. The values of the cantilever's force constant and the tip's radius are needed to transform the observables into forces. The thermal noise method ${ }^{329,330}$ in combination with an interferometric calibration of the optical sensitivity provides accurate calibration protocols of the force constant. ${ }^{83,331,332}$ Some of those protocols have been extended to the calibration of the force constants of higher modes. ${ }^{333,334}$

Similarly, several methods $\mathrm{s}^{335,336}$ have been proposed to determine the tip's radius. However, the resulting protocols do not offer a quick and non-destructive determination of the radius. An alternative approach to estimate the radius might be obtained from the spatial resolution of the images. This method is empirical and its accuracy relies on the identification of an intrinsic structure of the sample..$^{86,216}$

10.3.3 Sharp and colloidal tips. Nanomechanical mapping is about generating high-spatial resolution maps of mechanical properties. This definition demands the use of very sharp tips (sub-5 $\mathrm{nm}$ radii). Those tips are needed to reveal with nanoscale spatial resolution the mechanical properties of single proteins, protein patches or polymer surfaces.

Ideally the size of the tip should be smaller than the lateral extension of the key sample features. For single cells, the relevant features might lie in the sub-100 nm range. Therefore, nanomechanical mapping will require the use of tips with radii in the sub-20 nm range. ${ }^{67,337}$ The use colloidal tips with radii ranging from 1-50 $\mu \mathrm{m}$ is recommended for generating maps of the mechanical properties of tissues or decellularized extracellular matrices. ${ }^{85,338-340}$ In those samples the regions of interest might be several micrometers wide.

Accurate nanomechanical measurements require to process the data with the expression of the force that better describes the geometry of the tip (Table 1). For an isotropic material, the accuracy of the measurement is independent of the tip's size. The situation is more subtle on heterogeneous samples. Colloidal tips provide an average of the mechanical properties of the different structures or regions involved the contact area. Thus, experiments performed on different regions of the sample might yield similar values. On the other hand, a sharp tip will measure the mechanical properties of smaller regions. Repeating the measurements on other regions of the sample might yield significantly different values. The observed differences might reflect the variations of the local structure and properties of the sample and not errors associated with the sharpness of the tip.

\subsection{Comparisons with other methods}

The values of some nanomechanical maps were compared to the measurements obtained either by standard macroscopic techniques such as dynamic mechanical analysis (DMA) $)^{341,342}$ or by nanoindentation methods. ${ }^{142,343}$ In addition, there are also several comparative studies of AFM-based nanomechanical methods. ${ }^{344,345}$ To reach meaningful conclusions, comparative studies among macro, micro and nanoscale methods should always be accompanied by a discussion about modulation frequencies, calibration protocols, surface effects and micro and nanostructure of the sample.

10.4.1 Comparisons with macroscopic and nanoindentation measurements. AFM, nanoindentation and dynamic mechanical 
analysis (DMA) data are in good agreement when the differences in the experimental protocols are also included in the analysis. For example, similar values for the storage, loss modulus and loss tangent of several rubbers were measured by nanoscale rheology and DMA. ${ }^{108,316}$ The same conclusion was reached for FV measurements of the elastic, storage and loss tangent values of several polymers and polymers blends. ${ }^{86,346,347}$ One study performed on polyurethane and polystyrene samples reported that the agreement between rigidity modulus measured by FV and DMA macroscopic measurements was only possible for AFM experiments performed with dull tips $(R>800 \mathrm{~nm}) \cdot{ }^{348}$ This result is in conflict with the other studies that compared nanoscale with either micro-scale or macroscopic measurements. ${ }^{86,108,317,346,347,349}$

The stress applied on AFM-based measurements is between one and two orders of magnitude higher than the one applied on bulk measurements. It has been noted that, for certain materials, the stress applied on AFM could be in a nonlinear stress-strain regime, in particular when using sharp tips. ${ }^{348}$ This effect together with the use of continuous models to describe nanoscale properties might cause some discrepancies between nanoscale and bulk measurements. Paradoxically, the repeatability of AFM measurements and the overall agreement obtained between the data and the description provided by contact mechanics models and FEM simulations indicates that many AFM experiments are performed in a linear or near-linear elastic regime.

It should be noted that the value of a mechanical property at the nanoscale should not necessarily coincide with the value of the same property at the macroscale. Macroscale measurements provide average values over a large volume of the material. On the other hand, AFM measurements provide local values averaged over very small volumes of the material. Mechanical properties of heterogeneous surfaces might have intrinsic size effects, for example, the existence of stress gradients at the domain boundaries. ${ }^{350}$

10.4.2 Comparison among AFM-based nanomechanical methods. Comparative studies among nanomechanical force spectroscopy methods were performed by measuring the elastic and/or viscoelastic properties of a given sample. ${ }^{146,344,345}$ Those types of studies are empirical in nature. Therefore, the conclusions deduced from them should be taken tentatively unless the results are reasoned in terms of physical models or validated by tests against well-established calibration samples.

There is a strong activity in measuring the viscoelastic properties of soft matter by AFM-methods. ${ }^{52,88-97,351-353}$ This activity has generated several viscoelastic parameters which makes hard to perform direct comparisons among results from different groups even when the experiments were performed on similar materials. A convergence towards the same viscoelastic parameters should be expected for the next years.

\subsection{High-speed operation}

In the 90's, AFM images were typically acquired at a scanning rate in the fast direction of $1 \mathrm{~Hz}(0.01$ frame per s, $100 \times$ 100 pixels). In the 2010's the typical scanning rate was increased to $10 \mathrm{~Hz}$. Nowadays, high-speed AFM operation implies scanning rates above $100 \mathrm{~Hz}$ ( $\geq 1$ frame per s, $100 \times 100$ pixels).

The next generation of nanomechanical force spectroscopy methods will provide nanomechanical maps at high-speed rates. The compatibilities of AFM phase-imaging ${ }^{327}$ and bimodal $\mathrm{AFM}^{354}$ with high-speed operation has already been demonstrated.

\subsection{Correlative microscopy}

Optical microscopy remains the method of choice for the functional and compositional characterisation of biological specimens in situ. Optical microscopy images provide large fields of view, access to organelles deep inside the cell and molecular labelling. Nanomechanical mapping would benefit by its combination with different optical microscopy methods. At the tissue level, immunofluorescence could be applied to guide the AFM tip to specific regions of the sample. ${ }^{338,355}$ This combination provides a link between mechanical property characterization and biochemical composition. At the single cell level, the integration of super-resolution microscopy ${ }^{356}$ and nanomechanical mapping would offer unique features for understanding cell dynamics and function.

\subsection{Big data and Machine learning}

The potential of big data analysis and machine learning algorithms in AFM and force spectroscopy remains largely unexplored. $^{357-360}$ High-speed nanomechanical mapping will generate large data files that will require the use of optimized processing tools. At the same time, many technological and biomedical applications will demand the automatization of AFM imaging. Robotics, machine learning and new computational tools are expected to have a significant impact in the field.

\section{Conclusions}

This review presented the experimental approaches, the theoretical background and the capabilities of the most advanced nanomechanical mapping methods to measure at very high $(<10 \mathrm{~nm})$ or high-spatial resolution $(10-100 \mathrm{~nm})$ the mechanical properties of soft materials.

About 25 years ago the emergence of nanotechnology motivated the development of the first nanomechanical mapping methods. Nowadays, a variety of fields such as mechanobiology, the development of smart materials, energy storage, nanomedicine or clinical diagnosis provide the driving forces to expand the capabilities of nanomechanical force spectroscopy.

In the near future, the field will experience the incorporation of improved theoretical models and advanced data analysis tools. At the same time, new hardware components and architectures will make high-speed nanomechanical mapping routine. In the long-term, I envision a new generation of instruments that will integrate automatization, quantitative accuracy, nanoscale spatial resolution, correlative microscopy and large-scale characterization of soft materials in their native state and environment. 


\section{Conflicts of interest}

There are no conflicts to declare.

\section{Acknowledgements}

The author benefitted from multiple and insightful discussions with Simone Benaglia, Mingdong Dong, Victor G. Gisbert and Manuel R. Uhlig during the writing of this report. Careful reading of the manuscript by S.B., V. G. G. and M. R. Uhlig is also acknowledged. Financial support from the European Research Council ERC-AdG-340177 and the Ministerio de Ciencia e Innovación (MAT2016-76507-R, PID2019-106801GB-I00) are acknowledged.

\section{References}

1 G. Binnig, C. F. Quate and C. Gerber, Atomic force microscope, Phys. Rev. Lett., 1986, 56, 930.

2 E. L. Florin, V. T. Moy and H. E. Gaub, Adhesion forces between individual ligand-receptor pairs, Science, 1994, 264, 415.

3 F. Oesterhelt, D. Oesterhelt, M. Pfeiffer, A. Engel, H. E. Gaub and D. J. Muller, Unfolding pathways of individual bacteriorhodopsins, Science, 2000, 288, 143-146.

$4 \mathrm{~W}$. F. Heinz and J. H. Hoh, Spatially resolved force spectroscopy of biological surfaces using the atomic force microscope, Trends Biotechnol., 1999, 17, 143-150.

5 R. Garcia, R. Magerle and R. Perez, Nanoscale compositional mapping with gentle forces, Nat. Mater., 2007, 6, 405-411.

6 T. Ando, High-Speed Atomic Force Microscopy Coming of Age, Nanotechnology, 2012, 23, 062001.

7 D. Wang and T. P. Russell, Advances in Atomic Force Microscopy for Probing Polymer Structure and Properties, Macromolecules, 2018, 51, 3-24.

8 Y. F. Dufrêne, T. Ando, R. Garcia, D. Alsteens, D. MartinezMartin, A. Engel, C. Gerber and D. J. Müller, Imaging Modes of Atomic Force Microscopy for Application in Molecular and Cell Biology, Nat. Nanotechnol., 2017, 12, 295-307.

9 D. Sluysmans, S. Hubert, C. J. Bruns, Z. Zhu, J. F. Stoddart and A. S. Duwez, Synthetic oligorotaxanes exert high forces when folding under mechanical load, Nat. Nanotechnol., 2017, 13, 209-213.

10 A. Stylianou, S. V. Kontomaris, C. Grant and E. Alexandratou, Atomic force microscopy on biological materials related to pathological conditions, Scanning, 2019, 2019, 8452851.

11 N. Mandriota, C. Friedsam, J. A. Jones-Molina, K. Tatem, D. E. Ingber and O. Sahin, Cellular Nanoscale Stiffness Patterns Governed by Intracellular Forces, Nat. Mater., 2019, 18, 1071-1077.

12 D. E. Ingber, Mechanobiology and diseases of mechanotransduction, Ann. Med., 2003, 35, 564-577.
13 T. Fukuma and R. Garcia, Atomic- and Molecular-Resolution Mapping of Solid-Liquid Interfaces by 3D Atomic Force Microscopy, ACS Nano, 2018, 12, 11785-11797.

14 M. Radmacher, J. P. Cleveland, M. Fritz, H. G. Hansma and P. K. Hansma, Mapping interaction forces with the atomic force microscope, Biophys. J., 1994, 66, 2159-2165.

15 M. Radmacher, M. Fritz, C. M. Kacher, J. P. Cleveland and P. K. Hansma, Measuring the viscoelastic properties of human platelets with the atomic force microscope, Biophys. J., 1996, 70, 556-567.

16 S. N. Magonov and D. H. Reneker, Characterization of polymer surfaces with atomic force microscopy, Annu. Rev. Mater. Sci., 1997, 27, 175.

17 J. Tamayo and R. Garcia, Deformation, contact time and phase contrast in tapping mode scanning force microscopy, Langmuir, 1996, 12, 4430.

18 H. J. Butt, B. Cappella and M. Kappl, Force Measurements with the Atomic Force Microscope: Technique, Interpretation and Applications, Surf. Sci. Rep., 2005, 59, 1-152.

19 Y. F. Dufrene, D. Martinez-Martin, I. Medalsy, D. Alsteens and D. J. Muller, Multiparametric imaging of biological systems by force-distance curve-based AFM, Nat. Methods, 2013, 10, 847-854.

20 R. Garcia and E. T. Herruzo, The Emergence of Multifrequency Force Microscopy, Nat. Nanotechnol., 2012, 7, 217-226.

21 S. Zhang, H. Aslan, F. Besenbacher and M. Dong, Quantitative biomolecular imaging by dynamic nanomechanical mapping, Chem. Soc. Rev., 2014, 43, 7412-7429.

22 M. Chyasnavichyus, S. L. Young and V. V. Tsukruk, Recent Advances in Micromechanical Characterization of Polymer, Biomaterial, and Cell Surfaces with Atomic Force Microscopy, Jpn. J. Appl. Phys., 2015, 54, 08LA02.

23 J. P. Killgore and F. W. del Rio, Contact resonance force microscopy for viscoelastic property measurements: from fundamentals to state-of-the-art applications, Macromolecules, 2018, 51, 6977-6996.

24 S. Benaglia, V. G. Gisbert, A. P. Perrino, C. A. Amo and R. Garcia, Fast and High-Resolution Mapping of Elastic Properties of Biomolecules and Polymers with Bimodal AFM, Nat. Protoc., 2018, 13, 2890-2907.

25 A. Dazzi and C. Prater, AFM-IR: Technology and Applications in Nanoscale Infrared Spectroscopy and Chemical Imaging, Chem. Rev., 2017, 117, 5146-5173.

26 R. M. Overney, E. Meyer, J. Frommer, D. Brodbeck, R. Lüthi, L. Howald, H. J. Guntherodt, M. Fujihira, H. Takano and Y. Gotoh, Friction measurements on phase-separated thin films with a modified atomic force microscope, Nature, 1992, 359, 133-135.

27 C. A. Amo and R. Garcia, Fundamental High-Speed Limits in Single-Molecule, Single-Cell and Nanoscale Force Spectroscopies, ACS Nano, 2016, 10, 7117.

28 There are a variety of commercial names for force-volume software applications, among them, PeakForce QNM, FastForceMapping, PinPoint Nanomechanical mode or Quantitative Imaging QI. 
29 M. Stark, R. W. Stark, W. M. Heckl and R. Guckenberger, Inverting dynamic force microscopy: From signals to timeresolved interaction forces, Proc. Natl. Acad. Sci. U. S. A., 2002, 99, 8473-8478.

30 O. Sahin, S. Magonov, C. Su, C. F. Quate and O. Solgaard, An Atomic Force Microscope Tip Designed to Measure Time- Varying Nanomechanical Forces, Nat. Nanotechnol., 2007, 2, 507-514.

31 R. Garcia, Amplitude Modulation AFM, Wiley-VCH, Weinheim, Germany, 2010.

32 T. R. Rodriguez and R. Garcia, Compositional mapping of surfaces in atomic force microscopy by excitation of the second normal mode of the microcantilever, Appl. Phys. Lett., 2004, 84, 449-451.

33 U. Rabe and W. Arnold, Acoustic microscopy by atomic force microscopy, Appl. Phys. Lett., 1994, 64, 1493.

34 U. Rabe, K. Janser and W. Arnold, Vibrations of free and surface-coupled atomic force microscope cantilevers: Theory and experiment, Rev. Sci. Instrum., 1996, 67, 3281.

35 J. R. Lozano and R. Garcia, Theory of Phase Spectroscopy in Bimodal Atomic Force Microscopy, Phys. Rev. B: Condens. Matter Mater. Phys., 2009, 79, 1-9.

36 H. Li, Y. Chen and L. Dai, Concentrated-mass cantilever enhances multiple harmonics in tapping-mode atomic force microscopy, Appl. Phys. Lett., 2008, 92, 151903.

37 R. Garcia and A. San Paulo, Attractive and repulsive interaction regimes in tapping-mode atomic force microscopy, Phys. Rev. B: Condens. Matter Mater. Phys., 1999, 60, 4961.

38 R. Garcia and R. Perez, Dynamic AFM methods, Surf. Sci. Rep., 2002, 47, 197-302.

39 R. Garcia, C. J. Gomez, N. F. Martinez, S. Patile, C. Dietz and R. Magerle, Identification of Nanoscale Dissipation Processes by Dynamic Atomic Force Microscopy, Phys. Rev. Lett., 2006, 97, 016103.

40 J. E. Sader, T. Uchihashi, M. J. Higgins, A. Farrell, Y. Nakayama and S. P. Jarvis, Quantitative force measurements using frequency modulation atomic force microscopytheoretical foundations, Nanotechnology, 2005, 16, S94.

41 L. D. Landau, E. M. Lifshitz, A. M. Kosevich and L. P. Pitaevskii, Theory of Elasticity, Butterworth-Heinemann, Oxford, 3rd edn, 1986.

$42 \mathrm{~N}$. W. Tschoegl, The Phenomenological Theory of Linear Viscoelastic Behavior: An Introduction, Springer Berlin Heidelberg, Berlin, Heidelberg, 1989.

43 K. L. Johnson, Contact Mechanics, Cambridge University Press, Cambridge, 1985.

44 P. D. Garcia and R. Garcia, Determination of the Elastic Moduli of a Single Cell Cultured on a Rigid Support by Force Microscopy, Biophys. J., 2018, 114, 2923-2932.

45 J. Israelachvili, Intermolecular and Surface Forces, Elsevier Academic Press, London, 2005.

46 I. N. Sneddon, The relation between load and penetration in the axisymmetric Boussinesq problem for a punch of arbitrary profile, Int. J. Eng. Sci., 1965, 3, 47-57.

47 G. M. Pharr, W. C. Oliver and F. R. Brotzen, On the generality of the relationship among contact stiffness, contact area, and elastic modulus during indentation, J. Mater. Res., 1992, 7, 613-617.

48 E. H. Lee and J. R. M. Radok, The Contact Problem for Viscoelastic Bodies, J. Appl. Mech., 1960, 27, 438-444.

49 P. D. Garcia, C. R. Guerrero and R. Garcia, Time-Resolved Nanomechanics of a Single Cell under the Depolymerization of the Cytoskeleton, Nanoscale, 2017, 9, 12051-12059.

50 C. Zener, Elasticity and anelasticity of Metals, University of Chicago Press, Chicago, 1948.

51 P. Kollmannsberger and B. Fabry, Linear and Nonlinear Rheology of Living Cells, Annu. Rev. Mater. Res., 2011, 41, 75-97.

52 P. D. Garcia, C. R. Guerrero and R. Garcia, Nanorheology of living cells measured by AFM-based force-distance curves, Nanoscale, 2020, 12, 9133.

53 B. V. Derjaguin, V. M. Muller and Y. P. Toporov, Effect of contact deformations on adhesion of particles, J. Colloid Interface Sci., 1975, 53, 314-326.

54 N. Gavara, Combined strategies for optimal detection of the contact point in AFM force-indentation curves obtained on thin samples and adherent cells, Sci. Rep., 2016, 6, 21267.

55 P. Attard, Measurement and interpretation of elastic and viscoelastic properties with the atomic force microscope, J. Phys.: Condens. Matter, 2007, 19, 473201.

56 B. Rajabifar, J. M. Jadhav, D. Kiracofe, G. F. Meyers and A. Raman, Dynamic AFM on Viscoelastic Polymer Samples with Surface Forces, Macromolecules, 2018, 51, 9649-9661.

57 H. V. Guzman, P. D. Garcia and R. Garcia, Dynamic Force Microscopy Simulator (dForce): A Tool for Planning and Understanding Tapping and Bimodal AFM Experiments, Beilstein J. Nanotechnol., 2015, 6, 369-379.

$58 \mathrm{~J}$. Melcher, S. Hu and A. Raman, VEDA: A Web-Based Virtual Environment for Dynamic Atomic Force Microscopy, Rev. Sci. Instrum., 2008, 79, 061301.

59 T. J. R. Hughes, The finite element method: linear static and dynamic finite element analysis, Dover Publications Inc., New York, 2000.

60 R. Vargas-Pinto, H. Gong, A. Vahabikashi and M. Johnson, The Effect of the Endothelial Cell Cortex on Atomic Force Microscopy Measurements, Biophys. J., 2013, 105, 300-309.

61 S. Vichare, S. Sen and M. M. Inamdar, Cellular mechanoadaptation to substrate mechanical properties: contributions of substrate stiffness and thickness to cell stiffness measurements using AFM, Soft Matter, 2014, 10, 1174-1181.

62 C. Valero, B. Navarro, D. Navajas and J. M. García-Aznar, Finite element simulation for the mechanical characterization of soft biological materials by atomic force microscopy, J. Mech. Behav. Biomed. Mater., 2016, 62, 222-235.

63 J. R. Staunton, B. Doss, S. M. Lindsay and R. Ros, Correlating confocal microscopy and atomic force indentation reveals metastatic cancer cells stiffen during invasion into collagen I matrices, Sci. Rep., 2016, 6, 19686.

64 M. Galluzzi, G. Tang, C. S. Biswas, J. Zhao, S. Chen and F. J. Stadler, AFM methodology and AFMech suite software 
for nanomechanics on heterogenous soft materials, Nat. Commun., 2018, 9, 358464.

65 B. L. Doss, K. Rahmani Eliato, K. Lin and R. Ros, Quantitative Mechanical Analysis of Indentations on Layered, Soft Elastic Materials, Soft Matter, 2019, 15, 1776-1784.

66 Y. M. Efremov, M. Velay-Lizancos, C. J. Weaver, A. I. Athamneh, P. D. Zavattieri, D. M. Suter and A. Raman, Anisotropy vs Isotropy in Living Cell Indentation with AFM, Sci. Rep., 2019, 9, 1-12.

67 P. D. Garcia, C. R. Guerrero and R. Garcia, Subsurface Imaging of Cell Organelles by Force Microscopy, ACS Nano, 2019, 13, 9629-9637.

68 W. H. Roos, R. Bruinsma and G. J. L. Wuite, Physical virology, Nat. Phys., 2010, 6, 733.

69 C. Carrasco, A. Luque, M. Hernando-Pérez, R. Miranda, J. L. Carrascosa, P. A. Serena, M. de Ridder, A. Raman, J. Gómez-Herrero, I. A. T. Schaap, D. Reguera and P. J. de Pablo, Built-In Mechanical Stress in Viral Shell, Biophys. J., 2011, 100, 1100-1108.

70 C. Barth, A. S. Foster, C. R. Henry and A. L. Shluger, Recent Trends in Surface Characterization and Chemistry with High-Resolution Scanning Force Methods, Adv. Mater., 2011, 23, 477-501.

71 B. Reischl, M. Watkins and A. S. Foster, Free Energy Approaches for Modeling Atomic Force Microscopy in Liquids, J. Chem. Theory Comput., 2013, 9, 600-608.

72 Y. Sugimoto, A. Yurtsever, M. Abe, S. Morita, M. Ondracek, P. Pou and R. Pérez, Role of tip chemical reactivity on atom manipulation process in dynamic force microscopy, ACS Nano, 2013, 7, 7370-7376.

$73 \mathrm{X} . \mathrm{Hu}, \mathrm{W}$. Nanney, K. Umeda, T. Ye and A. Martini, Combined Experimental and Simulation Study of Amplitude Modulation Atomic Force Microscopy Measurements of Self-Assembled Monolayers in Water, Langmuir, 2018, 34, 9627-9633.

74 B. Luan and M. O. Robbins, Contact of single asperities with varying adhesion: comparing continuum mechanics to atomistic simulations, Phys. Rev. E: Stat., Nonlinear, Soft Matter Phys., 2006, 74, 02611.

75 J. G. Vilhena, et al., Albumin (BSA) Adsorption over Graphene in Aqueous Environment: Influence of Orientation, Adsorption Protocol, and Solvent Treatment, Langmuir, 2016, 32, 1742-1755.

76 J. G. Vilhena, A. C. Dumitru, E. T. Herruzo, J. I. MendietaMoreno, R. Garcia, P. A. Serena and R. Perez, Adsorption orientations and immunological recognition of antibodies on graphene, Nanoscale, 2016, 8, 13463-13475.

77 M. Martínez, C. D. Cooper, A. B. Poma and H. V. Guzman, Free energies of the disassembly of viral capsids from a multiscale molecular simulation approach, J. Chem. Inf. Model., 2019, 60, 974-981.

78 N. F. Martinez, W. Kaminski, C. J. Gomez, C. Albonetti, F. Biscarini, R. Perez and R. Garcia, Molecular scale energy dissipation in oligothiophene monolayers measured by dynamic force microscopy, Nanotechnology, 2009, 20, 434021.
79 F. Eghiaian, A. Rigato and S. Scheuring, Structural, Mechanical, and Dynamical Variability of the Actin Cortex in Living Cells, Biophys. J., 2015, 108, 1330-1340.

80 C. Kronlage, M. Schäfer-Herte, D. Böning, H. Oberleithner and J. Fels, Feeling for Filaments: Quantification of the Cortical Actin Web in Live Vascular Endothelium, Biophys. J., 2015, 109, 687-698.

81 M. Shibata, T. Uchibashi, T. Ando and R. Yasuda, Long-tip high-speed force microscopy for nanometer-scale imaging of live cells, Sci. Rep., 2015, 5, 8724.

82 E. Usukura, A. Narita, A. Yagi, S. Ito and J. Usukura, An unroofing method to observe the cytoskeleton directly at molecular resolution using atomic force microscopy, Sci. Rep., 2016, 6, 27472.

83 H. Schillers, et al., Standardized Nanomechanical Atomic Force Microscopy Procedure (SNAP) for Measuring Soft and Biological Samples, Sci. Rep., 2017, 7, 1-9.

84 C. T. Lim, E. H. Zhou and S. T. Quek, Mechanical models for living cells-a review, J. Biomech., 2006, 39, 195-216.

85 A. Dominguez-Bajo, A. González-Mayorga, C. R. Guerrero, F. J. Palomares, R. Garcia, E. Lopez-Dolado and M. C. Serrano, Myelinated axons and functional blood vessels populate mechanically compliant rGO foams in chronic cervical hemisected rats, Biomaterials, 2019, 192, 461-474.

86 E. T. Herruzo, A. P. Perrino and R. Garcia, Fast Nanomechanical Spectroscopy of Soft Matter, Nat. Commun., 2014, 5, 3126-3204.

87 H. F. Boyer, Atlas of stress-strain curves, ASM International, 2002.

88 P. H. Wu, D. R. B. Aroush, A. Asnacios, W. C. Chen, M. E. Dokukin, B. L. Doss, P. Durand-Smet, A. Ekpenyong, J. Guck, N. V. Guz, P. A. Janmey, J. S. H. Lee, N. M. Moore, A. Ott, Y. Poh, R. Ros, M. Sander, I. Sokolov, J. R. Staunton and N. Wang, et al., A Comparison of Methods to Assess Cell Mechanical Properties, Nat. Methods, 2018, 15, 491-498.

89 E. Moeendarbary, L. Valon, M. Fritzsche, A. R. Harris, D. A. Moulding, A. J. Thrasher, E. Stride, L. Mahadevan and G. T. Charras, The Cytoplasm of Living Cells Behaves as a Poroelastic Material, Nat. Mater., 2013, 12, 253-261.

90 J. Alcaraz, L. Buscemi, M. Grabulosa, X. Trepat, B. Fabry, R. Farré and D. Navajas, Microrheology of Human Lung Epithelial Cells Measured by Atomic Force Microscopy, Biophys. J., 2003, 84, 2071-2079.

91 F. M. Hecht, J. Rheinlaender, N. Schierbaum, W. H. Goldmann, B. Fabry and T. E. Schaffer, Imaging viscoelastic properties of live cells by AFM: power-law rheology on the nanoscale, Soft Matter, 2015, 11, 4584-4591.

92 92A. Rigato, A. Miyagi, S. Scheuring and F. Rico, HighFrequency Microrheology Reveals Cytoskeleton Dynamics in Living Cells, Nat. Phys., 2017, 13, 771-775.

93 B. R. Bruckner, H. Nöding and A. Janshoff, Viscoelastic Properties of Confluent MDCK II Cells Obtained from Force Cycle Experiments, Biophys. J., 2017, 112, 724-735.

94 J. S. De Sousa, J. A. C. Santos, E. B. Barros, L. M. R. Alencar, W. T. Cruz, M. V. Ramos and J. Mendes-Filho, Analytical 
Model of Atomic-Force-Microscopy Force Curves in Viscoelastic Materials Exhibiting Power Law Relaxation, J. Appl. Phys., 2017, 121, 034901.

95 P. D. Garcia and R. Garcia, Determination of the Viscoelastic Properties of a Single Cell Cultured on a Rigid Support by Force Microscopy, Nanoscale, 2018, 10, 19799-19809.

96 Y. M. Efremov, T. Okajima and A. Raman, Measuring viscoelasticity of soft biological samples using atomic force microscopy, Soft Matter, 2019, 16, 64-81.

97 J. S. de Sousa, R. S. Freire, F. D. Sousa, M. Radmacher, A. F. B. Silva, M. V. Ramos, A. C. O. Monteiro-Moreira, F. P. Mesquita, M. E. A. Moraes, R. C. Montenegro and C. L. N. Oliveira, Double power-law viscoelastic relaxation of living cells encodes motility trends, Sci. Rep., 2020, 10, 4749 .

98 D. Martínez-Martín, G. Fläschner, B. Gaub, S. Martin, R. Newton, C. Beerli and D. J. Muller, Inertial picobalance reveals fast mass fluctuations in mammalian cells, Nature, 2017, 550, 500-505.

99 E. K. Dimitriadis, F. Horkay, J. Maresca, B. Kachar and R. S. Chadwick, Determination of Elastic Moduli of Thin Layers of Soft Material Using the Atomic Force Microscope, Biophys. J., 2002, 82, 2798-2810.

100 N. Gavara and R. S. Chadwick, Determination of the elastic moduli of thin samples and adherent cells using conical AFM tips, Nat. Nanotechnol., 2012, 7, 733-736.

101 Y. Ding, J. Wang, G. K. Xu and G. F. Wang, Are elastic moduli of biological cells depth dependent or not? Another explanation using contact mechanics model with surface tension, Soft Matter, 2018, 14, 7434-7541.

102 T. C. T. Ting, The Contact Stresses Between a Rigid Indenter and a Viscoelastic Half-Space, J. Appl. Mech., 1966, 33, 845-854.

103 A. Rosa-Zeiser, E. Weilandt, S. Hild and O. Marti, The Simultaneous Measurement of Elastic, Electrostatic and Adhesive Properties by Scanning Force Microscopy: Pulsed - Force Mode Operation, Meas. Sci. Technol., 1997, 8, 1333-1338.

104 S. Krüger, D. Krüger and A. Janshoff, Scanning Force Microscopy Based Rapid Force Curve Acquisition on Supported Lipid Bilayers: Experiments and Simulations Using Pulsed Force Mode, ChemPhysChem, 2004, 5, 989-997.

105 J. Shi, Y. Hu, S. Hu, J. Ma and C. Su, Method and Apparatus of Using Peak Force Tapping Mode to Measure Physical Properties of a Sample, US Pat., 8650660B2, 2014.

106 B. Pettinger, N. Erina and C. Su, Quantitative mechanical property mapping at the nanoscale with PeakForce QNM, Bruker Application note, 2012, 128.

107 T. Igarashi, S. Fujinami, T. Nishi, N. Asao and K. Nakajima, Nanorheological Mapping of Rubbers by Atomic Force Microscopy, Macromolecules, 2013, 46, 1916-1922.

108 P. V. Kolluru, M. D. Eaton, D. W. Collinson, X. Cheng, D. E. Delgado, K. R. Shull and L. C. Brinson, AFM-based Dynamic Scanning Indentation (DSI) Method for Fast, High-resolution Spatial Mapping of Local Viscoelastic Properties in Soft Materials, Macromolecules, 2018, 51, 8964-8978.
109 D. Alsteens, V. Dupres, S. Yunus, J. P. Latgé, J. J. Heinisch and Y. F. Dufrêne, High-Resolution Imaging of Chemical and Biological Sites on Living Cells Using Peak Force Tapping Atomic Force Microscopy, Langmuir, 2012, 28, 16738-16744.

110 D. Alsteens, et al., Nanomechanical mapping of first binding steps of virus to animal cells, Nat. Nanotechnol., 2017, 12, 177.

111 I. Medalsy, U. Hensen and D. J. Muller, Imaging and Quantifying Chemical and Physical Properties of Native Proteins at Molecular Resolution by Force-Volume AFM, Angew. Chem., Int. Ed., 2011, 50, 12103-12108.

112 L. Wildling, B. Unterauer, R. Zhu, A. Rupprecht, T. Haselgrübler, C. Rank, A. Ebner, D. Vater, P. Pollheimer, E. Pohl, P. Hinterdorfer and H. J. Gruber, Linking of sensor molecules with amino groups to aminofunctionalized AFM tips, Bioconjugate Chem., 2011, 22, 1239-1248.

113 P. Hinterdorfer, W. Baumgartner, H. J. Gruber and K. Schilcher, Detection and Localization of Individual Antibody - Antigen Recognition Events by Atomic Force Microscopy, Proc. Natl. Acad. Sci. U. S. A., 1996, 93, 3477-3481.

114 C. D. Frisbie, L. F. Rozsnyai, A. Noy, M. S. Wrighton and C. M. Lieber, Functional group imaging by chemical force microscopy, Science, 1994, 265, 2071-2074.

115 A. Noy, D. V. Vezenov and C. M. Lieber, Chemical force microscopy, Annu. Rev. Mater. Sci., 1997, 27, 381-421.

116 D. Alsteens, D. J. Müller and Y. F. Dufrene, Multiparametric Atomic Force Microscopy Imaging of Biomolecular and Cellular Systems, Acc. Chem. Res., 2017, 50, 924-931.

117 A. Noy and R. W. Friddle, Practical Single Molecule Force Spectroscoopy: How to Determine Fundamental Thermodynamic Parameters of Intermolecular Bonds with an Atomic Force Microscope, Methods, 2013, 60, 142-150.

118 D. Alsteens, M. Pfreundschuh, C. Zhang, P. M. Spoerri, S. R. Coughlin, B. K. Kobilka and D. J. Muller, Imaging $\mathrm{G}$ protein-coupled receptors while quantifying their ligand-binding free-energy landscape, Nat. Methods, 2015, 12, 845-851.

119 M. Pfreundschuh, D. Harder, Z. Ucurum, D. Fotiadis and D. J. Müller, Detecting Ligand-Binding Events and Free Energy Landscape while Imaging Membrane Receptors at Subnanometer Resolution, Nano Lett., 2017, 17, 3261-3269.

120 E. Evans and K. Ritchie, Dynamic Strength of Molecular Adhesion Bonds, Biophys. J., 1997, 72, 1541-1555.

121 M. E. Dokukin and I. Sokolov, Nanoscale compositional mapping of cells, tissues, and polymers with ringing mode of atomic force microscopy, Sci. Rep., 2017, 7, 11828.

122 N. Kodera, D. Yamamoto, R. Ishikawa and T. Ando, Video Imaging of Walking Myosin $\mathrm{V}$ by High-Speed Atomic Force Microscopy, Nature, 2010, 468, 72-76.

123 F. Rico, L. Gonzalez, I. Casuso, M. Puig-Vidal and S. Scheuring, High-speed force spectroscopy unfolds titin at the velocity of molecular dynamics simulations, Science, 2013, 342, 741-743. 
124 A. P. Nievergelt, N. Banterle, S. H. Andany, P. Gonczy and G. E. Fantner, High-speed photothermal off-resonance atomic force microscopy reveals assembly routes of centriolar scaffold protein SAS-6, Nat. Nanotechnol., 2018, 13, 696.

125 M. R. Uhlig, C. A. Amo and R. Garcia, Dynamics of breaking intermolecular bonds in high-speed force spectroscopy, Nanoscale, 2018, 10, 17112.

126 L. M. Rebêlo, J. S. de Sousa, J. Mendes Filho, J. Schäpe, H. Doschke and M. Radmacher, Microrheology of cells with magnetic force modulation atomic force microscopy, Soft Matter, 2014, 10, 2141-2149.

127 M. Chyasnavichyus, S. L. Young and V. V. Tsukruk, Probing of Polymer Surfaces in the Viscoelastic Regime, Langmuir, 2014, 30, 10566.

128 Y. H. Chim, L. M. Mason, N. Rath, M. F. Olson, M. Tassieri and $\mathrm{H}$. Yin, A one-step procedure to probe the viscoelastic properties of cells by Atomic Force Microscopy, Sci. Rep., 2018, 8, 14462.

129 P. C. Nalam, N. N. Gosvami, M. A. Caporizzo, R. J. Composto and R. W. Carpick, Nanorheology of hydrogels using direct drive force modulation atomic force microscopy, Soft Matter, 2015, 11, 8165.

130 F. M. Hecht, J. Rheinlaender, N. Schierbaum, W. H. Goldmann, B. Fabry and T. E. Schäffer, Imaging viscoelastic properties of live cells by AFM: power-law rheology on the nanoscale, Soft Matter, 2015, 11, 4584.

131 S. D. Solares, Nanoscale Effects in the Characterization of Viscoelastic Materials with Atomic Force Microscopy: Coupling of a Quasi-Three-Dimensional Standard Linear Solid Model with in-Plane Surface Interactions, Beilstein J. Nanotechnol., 2016, 7, 554-571.

132 E. A. López-Guerra, B. Eslami and S. D. Solares, Calculation of standard viscoelastic responses with multiple retardation times through analysis of static force spectroscopy AFM data, J. Polym. Sci., Part B: Polym. Phys., 2017, 55, 804-813.

133 C. H. Parvini, M. A. S. R. Saadi and S. D. Solares, Extracting Viscoelastic Material Parameters using an Atomic Force Microscope and Static Force Spectroscopy, Beilstein J. Nanotechnol., 2020, 11, 922-937.

134 Y. M. Efremov, W. H. Wang, S. D. Hardy, R. L. Geahlen and A. Raman, Measuring Nanoscale Viscoelastic Parameters of Cells Directly from AFM Force-Displacement Curves, Sci. Rep., 2017, 7, 1-14.

135 Y. M. Efremov, A. I. Shpichka, S. L. Kotova and P. S. Timashev, Viscoelastic mapping of cells based on fast force volume and PeakForce Tapping, Soft Matter, 2019, 15, 5455-5463.

136 R. E. Mahaffy, C. K. Shih, F. C. MacKintosh and J. Käs, Scanning probe-based frequency-dependent microrheology of polymer gels and biological cells, Phys. Rev. Lett., 2000, 85, 880-883.

137 V. Vadillo-Rodriguez, T. J. Beveridge and J. R. Dutcher, Surface viscoelasticity of individual Gram-negative bacterial cells measured using atomic force microscopy, J. Bacteriol., 2008, 190, 4225-4232.

138 E. M. Darling, M. Topel, S. Zauscher, T. P. Vail and F. Guilak, Viscoelastic properties of human mesenchymally-derived stem cells and primary osteoblasts, chondrocytes, and adipocytes, J. Biomech., 2008, 41, 454-464.

139 S. Moreno-Flores, R. Benitez, M. D. M. Vivanco and J. L. Toca-Herrera, Stress Relaxation and Creep on Living Cells with the Atomic Force Microscope: A Means to Calculate Elastic Moduli and Viscosities of Cell Components, Nanotechnology, 2010, 21, 445101.

140 E. Ueda, X. Liang, M. Ito and K. Nakajima, Dynamic moduli mapping of silica-filled styrene-butadiene rubber vulanizate by nanorheological AFM, Macromolecules, 2019, 52, 311-319.

141 E. G. Herbet, W. C. Oliver and G. M. Pharr, Nanoindentation and dynamic characterization of viscoelastic solids, J. Phys. D: Appl. Phys., 2008, 41, 074021.

142 S. R. Cohen and E. Kalfon-Cohen, E. Dynamic Nanoindentation by Instrumented Nanoindentation and Force Microscopy: a Comparative Review, Beilstein J. Nanotechnol., 2013, 4, 815-833.

143 N. Schierbaum, J. Rheinlaender and T. E. Schäffer, Viscoelastic properties of normal and cancerous human breast cells are affected differently by contact to adjacent cells, Acta Biomater., 2017, 55, 239-248.

144 A. Voss, R. W. Stark and C. Dietz, Surface versus Volume Properties on the Nanoscale: Elastomeric Polypropylene, Macromolecules, 2014, 47, 5236-5245.

145 X. Meng, H. Zhang, J. Song, X. Fan and L. Sun, Broad modulus range nanomechanical mapping by magneticdrive soft probes, Nat. Commun., 2017, 8, 1944.

146 A. Bahrami, C. Bailly and B. Nysten, Spatial resolution and property contrast in local mechanical mapping of polymer blends using AFM dynamic force spectroscopy, Polymer, 2019, 165, 180-190.

147 M. Lorenzoni, L. Evangelio, S. Verhaeghe, N. C. Nicolet, C. Navarro and F. Pérez-Murano, Assessing the Local Nanomechanical Properties of Self - Assembled Block Copolymer Thin Films by Peak Force Tapping, Langmuir, 2015, 31, 11630-11638.

148 D. W. Collinson, M. D. Eaton, K. R. Shull and L. C. Brinson, Deconvolution of Stress Interaction Effects from Atomic Force Spectroscopy Data across Polymer-Particle Interfaces, Macromolecules, 2019, 52, 8940-8955.

149 P. M. Claesson, I. Dobryden, G. Li, Y. He, H. Huang, P. A. Thoren and D. B. Haviland, From force curves to surface nanomechanical properties, Phys. Chem. Chem. Phys., 2017, 19, 23642.

150 E. Limousin, D. E. Martinez-Tong, N. Ballard and J. M. Asua, Cure-Dependent Morphology of Acrylic/Alkyd Hybrid Latex Films via Nanomechanical Mapping, ACS Appl. Polym. Mater., 2019, 1, 2213-2223.

151 K. Hu, T. Wei, H. Li, C. He, H. Yang, T. P. Russell and D. Wang, Interfacial Broadening Kinetics between a Network and a Linear Polymer and Their Composites Prepared by Melt Blending, Macromolecules, 2019, 52, 9759-9765.

152 C. He, S. Sui, X. Wu, T. P. Russell and D. Wang, Atomic Force Microscopy Nanomechanical Mapping Visualizes Interfacial Broadening between Networks Due to Chemical 
Exchange Reactions, J. Am. Chem. Soc., 2018, 140, 6793-6796.

153 A. C. Dumitru, F. M. Espinosa, R. Garcia, G. Foschi, S. Tortorella, F. Valle, M. Dalavalle, F. Zerbetto and F. Biscarini, In situ nanomechanical characterization of the early stages of swelling and degradation of a biodegradable polymer, Nanoscale, 2018, 7, 5403-5410.

154 L. Wang, H. Wang, M. Wagner, Y. Yan, D. S. Jakob and $\mathrm{X}$. G. Xu, Nanoscale simultaneous chemical and mechanical imaging via peak force infrared microscopy, Sci. Adv., 2017, e1700255.

155 L. Li, P. Zhang, J. Li, Y. Wang, Y. Wei, J. Hu, X. Zhou, B. Xu and $\mathrm{B}$. Li, Measurement of nanomechanical properties of DNA molecules by PeakForce atomic force microscopy based on DNA origami, Nanoscale, 2019, 11, 4707-4711.

156 A. Bestembayeva, A. Kramer, A. A. Labokha, D. Osmanović, I. Liashkovich, E. V. Orlova, I. J. Ford, G. Charras, A. Fassati and B. W. Hoogenboom, Nanoscale stiffness topography reveals structure and mechanics of the transport barrier in intact nuclear pore complexes, Nat. Nanotechnol., 2015, 10, 60-64.

157 G. Lamour, A. Allard, J. Pelta, S. Labdi, M. Lenz and C. Campillo, Mapping and modeling the nanomechanics of bare and protein-coated lipid nanotubes, Phys. Rev. X, 2020, 10, 011031.

158 B. Gumí-Audenis, S. Illa-Tuset, N. Grimaldi, L. PasquinaLemonche, L. Ferrer-Tasies, F. Sanz, J. Veciana, I. Ratera, J. Faraudo, N. Ventosa and M. I. Giannotti, Insights into the structure and nanomechanics of a quatsome membrane by force spectroscopy measurements and molecular simulations, Nanoscale, 2018, 10, 23001-23011.

159 M. Marchetti, G. J. L. Wuite and W. H. Roos, Atomic force microscopy observation and characterization of single virions and virus-like particles by nano-indentation, Curr. Opin. Virol., 2016, 18, 82-88.

160 P. J. de Pablo and I. A. T. Schaap, Atomic force microscopy of viruses, Atomic Force Microscopy of Viruses, in Physical Virology, ed. U. Greber, Advances in Experimental Medicine and Biology, Springer, 2019, vol. 1215, pp. 159-179.

161 C. Braunsmann, J. Seifert, J. Rheinlaender and T. Schaffer, High-speed force mapping on living cells with a small cantilever atomic force microscope, Rev. Sci. Instrum., 2014, 85, 073703.

162 A. Rigato, F. Rico, F. Eghiaian, M. Piel and S. Scheuring, Atomic Force Microscopy Mechanical Mapping of Micropatterned Cells Shows Adhesion Geometry-Dependent Mechanical Response on Local and Global Scales, ACS Nano, 2015, 9, 5846-5856.

163 K. Beicker, E. T. O’Brien, M. R. Falvo and R. Superfine, Vertical Light Sheet Enhanced Side-View Imaging for AFM Cell Mechanics Studies, Sci. Rep., 2018, 8, 1504.

164 A. Calzado-Martín, M. Encinar, J. Tamayo, M. Calleja and A. San Paulo, Effect of Actin Organization on the Stiffness of Living Breast Cancer Cells Revealed by Peak-Force Modulation Atomic Force Microscopy, ACS Nano, 2016, 10, 3365-3374.
165 S. Karsch, D. Kong, J. Großhans and A. Janshoff, SingleCell Defects Cause a Long-Range Mechanical Response in a Confluent Epithelial Cell Layer, Biophys. J., 2017, 113, 2601-2608.

166 A. C. Dumitru, M. A. Poncin, L. Conrard and Y. F. Dufrêne, Nanoscale membrane architecture of healthy and pathological red blood cells, Nanoscale Horiz., 2018, 3, 293-304.

167 S. J. Baldwin, L. Kreplak and J. M. Lee, Characterization via atomic force microscopy of discrete plasticity in collagen fibrils from mechanically overloaded tendons: Nano-scale structural changes mimic rope failure, J. Mech. Behav. Biomed. Mater., 2016, 60, 356-366.

168 C. Formosa-Daguea, P. Spezialeb, T. J. Fosterc, J. A. Geogheganc and Y. F. Dufrêne, Zinc-dependent mechanical properties of Staphylococcus aureus biofilm-forming surface protein SasG, Proc. Natl. Acad. Sci. U. S. A., 2016, 113, 410-415.

169 H. Marshall, S. Aguayo, M. Kilian, F. Petersen, L. Bozec and J. Brown, In-Vivo Relationship between the NanoBiomechanical Properties of Streptococcal Polysaccharide Capsules and Virulence Phenotype, ACS Nano, 2020, 14, 1070-1083.

170 L. Stühn, A. Fritschen, J. Choy, M. Dehnert and C. Dietz, Nanomechanical sub-surface mapping of living biological cells by force microscopy, Nanoscale, 2019, 11, 13089-13097.

171 N. Barois, E. Werkmeister, S. Divoux, F. Perez and F. Lafont, Stiffness tomography of eukaryotic intracellular compartments by atomic force microscopy, Nanoscale, 2019, 11, 10320-10328.

172 A. G. Onaran, M. Balantekin, W. Lee, W. L. Hughes, B. A. Buchine, R. O. Guldiken, Z. Parlak, C. F. Quate and F. L. Degertekin, A new atomic force microscope probe with force sensing integrated readout and active tip, Rev. Sci. Instrum., 2006, 77, 023501.

173 M. Balantekin, A. G. Onaran and F. L. Degertekin, Quantitative mechanical characterization of materials at the nanoscale through direct measurement of time-resolved tip-sample interaction forces, Nanotechnology, 2008, 19, 085704.

174 A. F. Sarioglu and O. Solgaard, Cantilevers with integrated sensor for time-resolved force measurements in tappingmode atomic force microscopy, Appl. Phys. Lett., 2008, 93, 023114.

175 A. F. Sarioglu, S. Magonov and O. Solgaard, Tapping-mode force spectroscopy using cantilevers with interferometric high-bandwidth force sensors, Appl. Phys. Lett., 2012, 100, 053109.

176 J. Legleiter, M. Park, B. Cusick and T. Kowalewski, Scanning Probe Acceleration Microscopy (SPAM) in Fluids: Mapping Mechanical Properties of Surfaces at the Nanoscale, Proc. Natl. Acad. Sci. U. S. A., 2006, 103, 4813-4818.

177 M. H. Lee and W. H. Jhe, General Theory of AmplitudeModulation Atomic Force Microscopy, Phys. Rev. Lett., 2006, 97, 036104.

178 H. Holscher, Quantitative Measurement of Tip-Sample Interactions in Amplitude Modulation Atomic Force Microscopy, Appl. Phys. Lett., 2006, 89, 123109. 
179 A. J. Katan, M. H. van Es and T. H. Oosterkamp, Quantitative Force Versus Distance Measurements in Amplitude Modulation AFM: a Novel Force Inversion Technique, Nanotechnology, 2009, 20, 165703.

180 D. Platz, D. Forchheimer, E. A. Tholén and D. B. Haviland, Interaction imaging with amplitude-dependence force spectroscopy, Nat. Commun., 2013, 4, 1360.

181 G. Saraswat, P. Agarwal, G. Haugstad and M. V. Salapaka, Real-time probe based quantitative determination of material properties at the nanoscale, Nanotechnology, 2013, 24, 265706.

182 A. F. Payam, D. Martin-Jimenez and R. Garcia, Force reconstruction from tapping mode force microscopy experiments, Nanotechnology, 2015, 26, 1-12.

183 E. Meyer, H. J. Hug and R. Bennewitz, Scanning Probe Microscopy, Lab on a tip, Springer-Verlag, Berlin, 2004.

184 O. Sahin and N. Erina, High-resolution and large dynamic range nanomechanical mapping in tapping-mode atomic force microscopy, Nanotechnology, 2008, 19, 445717.

185 A. Wang, K. Vijayraghavan, O. Solgaard and M. J. Butte, Fast Stiffness Mapping of Cells Using High-Bandwidth Atomic Force Microscopy, ACS Nano, 2016, 10, 257-264.

186 N. Shamitko-Klingensmith, K. M. Molchanoff, K. A. Burke, G. J. Magnone and J. Legleiter, Mapping the mechanical properties of cholesterol-containing supported lipid bilayers with nanoscale spatial resolution, Langmuir, 2012, 28, 13411-13422.

187 T. R. Rodriguez and R. Garcia, Tip motion in amplitude modulation (tapping-mode) atomic-force microscopy: Comparison between continuous and point-mass models, Appl. Phys. Lett., 2002, 80, 1646.

188 R. W. Stark, Spectroscopy of higher harmonics in dynamic atomic force microscopy, Nanotechnology, 2004, 15, 347.

189 V. Vahdat and R. W. Carpick, Practical Method to Limit Tip-Sample Contact Stress and Prevent Wear in Amplitude Modulation Atomic Force Microscopy, ACS Nano, 2013, 7, 9836-9850.

190 A. San Paulo and R. Garcia, High-resolution imaging of antibodies by tapping-mode atomic force microscopy: Attractive and repulsive tip-sample interaction regimes, Biophys. J., 2000, 78, 1599.

191 A. P. Perrino and R. Garcia, How Soft Is a Single Protein? The Stress-strain Curve of Antibody Pentamers with 5 pN and 50 pm Resolutions, Nanoscale, 2016, 8, 9151-9158.

192 M. Penedo and H. J. Hug, Off-resonance intermittent contact mode multi-harmonic scanning force microscopy, Appl. Phys. Lett., 2018, 113, 023103.

193 O. Sahin, G. Yaralioglu, R. Grow, S. F. Zappe, A. Atalar, C. Quate and O. Solgaard, High-resolution imaging of elastic properties using harmonic cantilevers, Sens. Actuators, $A, 2004,114,183$.

194 Y. Hou, C. Ma, W. Wang and Y. Chen, Binary coded cantilevers for enhancing multi-harmonic atomic force microscopy, Sens. Actuators, A, 2019, 300, 111668.

195 A. Keyvani, H. Sadeghian, M. S. Tamer, J. F. LoodewijkGoosen and F. van Keulen, Minimizing tip-sample forces and enhancing sensitivity in atomic force microscopy with dynamically compliant cantilevers, J. Appl. Phys., 2017, 121, 244505.

196 O. Sahin, Time-varying tip-sample force measurements and steady-state dynamics in tapping-mode atomic force microscopy, Phys. Rev. B: Condens. Matter Mater. Phys., 2008, 77, 115405.

197 M. Dong, S. Husale and O. Sahin, Determination of Protein Structural Flexibility by Microsecond Force Spectroscopy, Nat. Nanotechnol., 2009, 4, 514-517.

198 L. Liu, Q. Li, S. Zhang, X. Wang, S. Hoffmann, J. Li, Z. Liu, F. Besenbacher and M. D. Dong, Identification of a Novel Parallel $\beta$-Strand Conformation within Molecular Monolayer of Amyloid Peptide, Adv. Sci., 2016, 3, 1500369.

199 M. Dong and O. Sahin, A nanomechanical interface to rapid single-molecule interactions, Nat. Commun., 2011, $2,247$.

200 Z. Jiang, S. Zhang, L. H. Klausen, J. Song, Q. Li, Z. Wang, B. T. Stokke, Y. Huang, F. Besenbacher, L. P. Nielsen and M. Dong, In vitro single-cell dissection revealing the interior structure of cable bacteria, Proc. Natl. Acad. Sci. U. S. A., 2018, 115, 8517-8522.

201 R. Garcia, Intracellular forces from stiffness, Nat. Mater., 2019, 18, 1037-1038.

202 S. Husale, H. J. Persson and O. Sahin, DNA nanomechanics allows direct digital detection of complementary DNA and microRNA targets, Nature, 2009, 462, 1075-1078.

203 H. Huang, I. Dobryden and P. A. Thoren, et al., Local surface mechanical properties of PDMS-silica nanocomposite probed with Intermodulation AFM, Compos. Sci. Technol., 2017, 150, 111-119.

204 D. Martin-Jimenez, E. Chacon, P. Tarazona and R. Garcia, Atomically resolved three-dimensional structures of electrolyte aqueous solutions near a solid surface, Nat. Commun., 2016, 7, 12164.

205 M. R. Uhlig, D. Martin-Jimenez and R. Garcia, Atomic-scale mapping of hydrophobic layers on graphene and few-layer $\mathrm{MoS}_{2}$ and $\mathrm{WSe}_{2}$ in water, Nat. Commun., 2019, 10, 2606.

206 C. M. Stroh, H. Wang, R. Bash, B. Ashcroft, J. Nelson, H. Gruber, D. Lohr, S. M. Lindsay and P. Hinterdorfer, Single-molecule recognition imaging microscopy, Proc. Natl. Acad. Sci. U. S. A., 2004, 101, 12503.

207 J. Preiner, A. Ebner, L. Chtcheglova, R. Zhu and P. Hinterdorfer, Simultaneous topography and recognition imaging: physical aspects and optimal imaging conditions, Nanotechnology, 2009, 20, 215103.

208 J. Preiner, N. S. Losilla, A. Ebner, P. Annibale, F. Biscarini, R. Garcia and P. Hinterdorfer, Imaging and detection of single molecule recognition events on organic semiconductor surfaces, Nano Lett., 2009, 9, 571.

209 Y. J. Oh, M. Koehler, Y. Lee, S. Mishra, J. W. Park and P. Hinterdorfer, Ultra-Sensitive and Label-Free Probing of Binding Affinity Using Recognition Imaging, Nano Lett., 2019, 19, 612-617.

210 M. Koehler, A. Fis, H. J. Gruber and P. Hinterdorfer, AFMBased Force Spectroscopy Guided by Recognition Imaging: 
A New Mode for Mapping and Studying Interaction Sites at Low Lateral Density, Methods Protoc., 2019, 2, 6.

211 R. Garcia and R. Proksch, Nanomechanical Mapping of Soft Matter by Bimodal Force Microscopy, Eur. Polym. J., 2013, 49, 1897-1906.

212 C. Dietz, E. T. Herruzo, J. R. Lozano and R. Garcia, Nanomechanical coupling enables detection and imaging of $5 \mathrm{~nm}$ superparamagnetic particles in liquid, Nanotechnology, 2011, 22, 125708.

213 C. Y. Lai, S. Santos and M. Chiesa, Systematic Multidimensional Quantification of Nanoscale Systems from Bimodal Atomic Force Microscopy Data, ACS Nano, 2016, 10, 6265-6272.

214 C. A. Amo, A. P. Perrino, A. F. Payam and R. Garcia, Mapping Elastic Properties of Heterogeneous Materials in Liquid with Angstrom-Scale Resolution, ACS Nano, 2017, 11, 8650.

215 M. Kocun, A. Labuda, W. Meinhold, I. Revenko and R. Proksch, Fast, High Resolution, and Wide Modulus Range Nanomechanical Mapping with Bimodal Tapping Mode, ACS Nano, 2017, 11, 10097-10105.

216 S. Benaglia, C. A. Amo and R. Garcia, Fast, quantitative and high resolution mapping of viscoelastic properties with bimodal AFM, Nanoscale, 2019, 11, 15289-15297.

217 S. D. Solares and G. Chawla, Triple-frequency intermittent contact atomic force microscopy characterization: Simultaneous topographical, phase, and frequency shift contrast in ambient air, J. Appl. Phys., 2010, 108, 054901.

218 S. Kawai, T. Glatzel, S. Koch, B. Such, A. Baratoff and E. Meyer, Ultrasensitive Detection of Lateral Atomic-Scale Interactions on Graphite (0001) via Bimodal Dynamic Force Measurements, Phys. Rev. B: Condens. Matter Mater. Phys., 2010, 81, 085420.

219 C. Dietz, Sensing In-Plane Nanomechanical Surface and Sub-Surface Properties of Polymers: Local Shear Stress as Function of the Indentation Depth, Nanoscale, 2018, 10, 460-468.

220 Y. Naitoh, R. Turanský, J. Brndiar, Y. J. Li, I. Štich and Y. Sugawara, Subatomic-Scale Force Vector Mapping above a Ge(001) Dimer Using Bimodal Atomic Force Microscopy, Nat. Phys., 2017, 13, 663-667.

221 J. R. Lozano and R. Garcia, Theory of Multifrequency Atomic Force Microscopy, Phys. Rev. Lett., 2008, 100, 8-11.

222 S. Kawai, T. Glatzel, S. Koch, B. Such, A. Baratoff and E. Meyer, Systematic Achievement of Improved AtomicScale Contrast via Bimodal Dynamic Force Microscopy, Phys. Rev. Lett., 2009, 103, 220801.

223 A. Labuda, M. Kocun, W. Meinhold, D. Walters and R. Proksch, Generalized Hertz Model for Bimodal Nanomechanical Mapping, Beilstein J. Nanotechnol., 2016, 7, 970-982.

224 R. Proksch, Multifrequency, repulsive-mode amplitudemodulated atomic force microscopy, Appl. Phys. Lett., 2006, 89, 1-4.

225 N. F. Martinez, S. Patil, J. R. Lozano and R. Garcia, Enhanced Compositional Sensitivity in Atomic Force
Microscopy by the Excitation of the First Two Flexural Modes, Appl. Phys. Lett., 2006, 89, 153115.

226 D. Ebeling, B. Eslami and S. D. Solares, Visualizing the subsurface of soft matter: Simultaneous topographical imaging, depth modulation, and compositional mapping with triple frequency atomic force microscopy, ACS Nano, 2013, 7, 10387-10396.

227 S. D. Solares, Challenges and complexities of multifrequency atomic force microscopy in liquid environments, Beilstein J. Nanotechnol., 2014, 5, 298-307.

228 S. M. Ang, S. D. Solares, S. Santos and D. Ebeling, Energy transfer between eigenmodes in multimodal atomic force microscopy, Nanotechnology, 2014, 25, 47501.

229 B. Eslami and M. Damircheli, Biharmonic versus bimodal AFM: Numerical and experimental study on soft matter, J. Appl. Phys., 2019, 126, 09530.

230 M. Damircheli and B. Eslami, Enhancing phase contrast for bimodal AFM imaging in low quality factor environments, Ultramicroscopy, 2019, 204, 18-26.

231 C. Dietz, M. Schulze, A. Voss, C. Riesch and R. W. Stark, Bimodal frequency-modulated atomic force microscopy with small cantilevers, Nanoscale, 2015, 7, 1849-1856.

232 D. Forchheimer, R. Forchheimer and D. B. Haviland, Improving image contrast and material discrimination with nonlinear response in bimodal atomic force microscopy, Nat. Commun., 2015, 6, 6270.

233 C. Y. Lai, V. Barcons, S. Santos and M. Chiesa, Periodicity in bimodal atomic force microscopy, J. Appl. Phys., 2015, 118, 044905.

234 M. G. Ruppert and S. O. R. Moheimani, High-bandwidth multimode self-sensing in bimodal atomic force microscopy, Beilstein J. Nanotechnol., 2016, 7, 284-295.

235 D. S. Wastl, A. J. Weymouth, T. Arai and F. J. Giessibl, Amplitude dependence of image quality in atomicallyresolved bimodal atomic force microscopy, Appl. Phys. Lett., 2016, 109, 141603.

236 S. Shi, D. Guo and J. Luo, Enhanced phase and amplitude image contrasts of polymers in bimodal atomic force microscopy, RSC Adv., 2017, 7, 11768-11776.

237 A. Bubendorf, S. Walheim, T. Schimmel and E. Meyer, A robust AFM-based method for locally measuring the elasticity of samples, Beilstein J. Nanotechnol., 2018, 9, 1-10.

238 R. Garcia, V. G. Gisbert and C. A. Amo, Bimodal method and system for quantifying long-range interactions and properties in atomic force microscopy, PCT/ES2020/ 070154.

239 A. Knoll, R. Magerle and G. Krausch, Tapping Mode Atomic Force Microscopy on Polymers: Where Is the True Sample Surface?, Macromolecules, 2001, 34, 4159-4165.

240 D. J. Muller and A. Engel, The height of biomolecules measured with the atomic force microscope depends on electrostatic interactions, Biophys. J., 1997, 73, 1633-1644.

241 S. Santos, V. Barcons, H. K. Cristenson, J. Font and N. H. Thomson, The Intrinsic Resolution Limit in the Atomic Force Microscope: Implications for Heights of Nano-Scale Features, PLoS One, 2011, 6, e23821. 
242 R. Giridharagopal, L.-Q. Flagg, J. S. Harrison, M. E. Ziffer, J. Onorato, C. K. Luscombe and D. S. Ginger, Electrochemical Strain Microscopy Probes Morphology-Induced Variations in Ion Uptake and Performance in Organic Electrochemical Transistors, Nat. Mater., 2017, 16, 1-6.

243 V. Shalabaeva, A. C. Bas, M. Piedrahita-Bello, K. Ridier, L. Salmon, C. Thibault, W. Nicolazzi, G. Molnár and A. Bousseksou, Direct Visualization of Local Spin Transition Behaviors in Thin Molecular Films by Bimodal AFM, Small, 2019, 15, 1903892.

244 E. N. Athanasopoulou, N. Nianias, Q. K. Ong and F. Stellacci, Bimodal Atomic Force Microscopy for the Characterization of Thiolated Self-Assembled Monolayers, Nanoscale, 2018, 10, 23027-23036.

245 H. K. Nguyen, M. Ito and K. Nakajima, Elastic and Viscoelastic Characterization of Inhomogeneous Polymers by Bimodal Atomic Force Microscopy, Jpn. J. Appl. Phys., 2016, 55, 08NB06.

246 M. F. Dupont, A. Elbourne and E. Mayes, Measuring the mechanical properties of flexible crystals using bi-modal atomic force microscopy, Phys. Chem. Chem. Phys., 2019, 21, 20219-20224.

247 Y. Li, C. Yu, Y. Gan, P. Jiang, J. Yu, Y. Ou, D. F. Zou, C. Huang, J. Wang, T. Jia, Q. Luo, X. F. Yu, H. Zhao, C. F. Gao and J. Li, Mapping the Elastic Properties of TwoDimensional $\mathrm{MoS}_{2}$ via Bimodal Atomic Force Microscopy and Finite Element Simulation, npj Comput. Mater., 2018, 4, 49.

248 J. Y. Lu, T. Olukan and S. R. Tamalampudi, Insights into graphene wettability transparency by locally probing its surface free energy, Nanoscale, 2019, 11, 7944-7951.

249 Z. Al-Rekabi and S. Contera, Multifrequency AFM Reveals Lipid Membrane Mechanical Properties and the Effect of Cholesterol in Modulating Viscoelasticity, Proc. Natl. Acad. Sci. U. S. A., 2018, 115, 2658-2663.

250 W. Trewby, J. Faraudo and K. Voïtchovsky, Long-Lived Ionic Nano-Domains Can Modulate the Stiffness of Soft Interfaces, Nanoscale, 2019, 11, 4376-4384.

251 M. E. F. Brollo, A. Dominguez-Bajo, A. Tabero, V. DomínguezArca, V. G. Gisbert, G. Prieto, C. Johansson, R. Garcia, A. Villanueva, M. C. Serrano and M. P. Morales, Combined Magnetoliposome Formation and Drug Loading in One Step for Efficient AC-Magnetic Field Remote Controlled Drug Release, ACS Appl. Mater. Interfaces, 2020, 12, 4295-4307.

252 G. Lamour, C. K. Yip, H. Li and J. Gsponer, High Intrinsic Mechanical Flexibility of Mouse Prion Nanofibrils Revealed by Measurements of Axial and Radial Young's Moduli, ACS Nano, 2014, 8, 3851-3861.

253 J. Gilbert, M. Charnley, C. Cheng and N. P. Reynolds, Quantifying Young's moduli of protein fibrils and particles with bimodal force spectroscopy, Biointerphases, 2017, 12, 041001.

254 K. Bose, C. J. Lech, B. Heddi and A. T. Phan, Highresolution AFM structure of DNA G-wires in aqueous solution, Nat. Commun., 2018, 9, 1959.

255 Y. Sun, L. H. Vu, N. Chew, Z. Puthucheary, M. E. Cove and K. A. Zeng, Study of Perturbations in Structure and Elastic
Modulus of Bone Microconstituents Using Bimodal Amplitude Modulated-Frequency Modulated Atomic Force Microscopy, ACS Biomater. Sci. Eng., 2019, 5, 478-486.

256 I. González-Domínguez, S. Gutiérrez-Granados, L. Cervera, F. Gòdia and N. Domingo, Identification of HIV-1-Based Virus-like Particles by Multifrequency Atomic Force Microscopy, Biophys. J., 2016, 111, 1173-1179.

257 A. X. Cartagena-Rivera, W. H. Wang, R. L. Geahlen and A. Raman, Fast, Multi-Frequency, and Quantitative Nanomechanical Mapping of Live Cells Using the Atomic Force Microscope, Sci. Rep., 2015, 5, 1-11.

258 D. Guan, E. Charlaix, R. Z. Qi and P. Tong, Noncontact Viscoelastic Imaging of Living Cells Using a Long-Needle Atomic Force Microscope with Dual-Frequency Modulation, Phys. Rev. Appl., 2017, 8, 1-6.

259 B. J. Rodriguez, C. Callahan, S. V. Kalinin and R. Proksch, Dual-frequency resonance-tracking atomic force microscopy, Nanotechnology, 2007, 18, 475504.

260 D. Nowak, W. Morrison1, H. K. Wickramasinghe, J. Jahng, E. Potma, L. Wan, R. Ruiz, T. R. Albrecht, K. Schmidt, J. Frommer, D. P. Sanders and S. Park, Nanoscale chemical imaging by photoinduced force microscopy, Sci. Adv., 2016, 2, e1501571.

261 A. Ambrosio, R. C. Devlin, F. Capasso and W. L. Wilson, Observation of Nanoscale Refractive Index Contrast via Photoinduced Force Microscopy, ACS Photonics, 2017, 4, 846-851.

262 M. Penedo, S. Hormeño, P. Prieto, R. Alvaro, J. Anguita, F. Briones and M. Luna, Selective enhancement of individual cantilever high resonance modes, Nanotechnology, 2015, 26, 485706.

263 A. B. Arı, M. Ç. Karakan, C. Yanık, İ. İ. Kaya and M. S. Hanay, Intermodal coupling as a probe for detecting nanomechanical modes, Phys. Rev. Appl., 2018, 9, 034024.

264 R. Potekin, S. Dharmasen, H. Keuma, X. Jiang, J. Lee, S. Kim, L. A. Bergman, A. F. Vakakis and H. Cho, Multifrequency Atomic Force Microscopy based on enhanced internal resonance of an inner-paddled cantilever, Sens. Actuators, A, 2018, 273, 206-220.

265 D. M. Harcombe, M. G. Ruppert, M. R. P. Ragazzon, S. O. Reza Moheimani and A. J. Flemming, A review of demodulation techniques for multifrequency atomic force microscopy, Beilstein J. Nanotechnol., 2020, 11, 76-91.

266 U. Rabe, S. Amelio, M. Kopycinska, S. Hirsekorn, M. Kempf, M. Göken and W. Arnold, Imaging and measurement of local mechanical material properties by atomic force acoustic microscopy, Surf. Interface Anal., 2002, 33, 65-70.

267 A. Raman, S. Trigueros, A. Cartagena, A. P. Z. Stevenson, M. Susilo, E. Nauman and S. A. Contera, Mapping Nanomechanical Properties of Live Cells Using Multi-Harmonic Atomic Force Microscopy, Nat. Nanotechnol., 2011, 6, 809-814.

268 M. R. P. Ragazzon, J. T. Gravdahl and K. Y. Pettersen, Model-Based Identification of Nanomechanical Properties in Atomic Force Microscopy, IEEE Trans. Control Syst. Technol., 2019, 27, 2045-2047. 
269 A. Briggs, G. A. D. Briggs and O. V. Kolosov, Acoustic microscopy, Oxford University Press, 2010.

270 G. Stan and S. D. Solares, Frequency, amplitude, and phase measurements in contact resonance atomic force microscopies, Beilstein J. Nanotechnol., 2014, 5, 278-288.

271 P. A. Yuya, D. C. Hurley and J. A. Turner, Contactresonance atomic force microscopy for viscoelasticity, J. Appl. Phys., 2008, 104, 074916.

272 J. P. Killgore, D. G. Yablon, A. H. Tsou, A. Gannepalli, P. A. Yuya, J. A. Turner, R. Proksch and D. C. Hurley, Viscoelastic Property Mapping with Contact Resonance Force Microscopy, Langmuir, 2011, 27, 13983-13987.

273 G. Stan, S. D. Solares, B. Pittenger, N. Erina and C. Su, Nanoscale mechanics by tomographic contact resonance atomic force microscopy, Nanoscale, 2014, 6, 962-969.

274 D. Passeri, A. Bettucci, M. Germano, M. Rossi, A. Alippi, S. Orlanducci, M. L. Terranova and M. Ciavarella, Effect of tip geometry on local indentation modulus measurement via atomic force acoustic microscopy technique, Rev. Sci. Instrum., 2005, 76, 093904.

275 G. S. Shekhawat and V. P. Dravid, Nanoscale imaging of buried structures via scanning near-filed ultrasound holography, Science, 2005, 310, 89.

276 L. Tetard, A. Passian and T. Thundat, New Modes for Subsurface Atomic Force Microscopy through Nanomechanical Coupling, Nat. Nanotechnol., 2010, 5, 105-109.

277 P. Vitry, R. Rebois, E. Bourillot, A. Deniset-Besseau, M. J. Virolle, E. Lesniewska and A. Dazzi, Combining infrared and mode synthesizing atomic force microscopy: Application to the study of lipid vesicles inside Streptomyces bacteria, Nano Res., 2016, 9, 1674-1681.

278 M. Soliman, J. Ding and L. Tetard, Nanoscale subsurface imaging, J. Phys.: Condens. Matter, 2017, 29, 173001.

279 R. Garcia, Probe microscopy: Images from below the surface, Nat. Nanotechnol., 2010, 5, 101-102.

280 G. J. Verbiest and M. J. Rost, Beating beats mixing in heterodyne detection schemes, Nat. Commun., 2015, 6, 6444.

281 G. J. Verbiest, J. N. Simon, T. H. Oosterkamp and M. J. Rost, Subsurface atomic force microscopy: towards a quantitative understanding, Nanotechnology, 2012, 23, 145704.

282 Y. M. Efremov, A. X. Cartagena-Rivera, A. I. M. Athamneh, D. M. Suterr and A. Raman, Mapping heterogeneity of cellular mechanics by multi-harmonic atomic force microscopy, Nat. Protoc., 2018, 13, 2200-2216.

283 N. H. Shaik, R. G. Reifenberger and A. Raman, Nanomechancial mapping in air or vacuum using mult-harmonic signals in tapping mode AFM, Nanotechnology, 2020, DOI: 10.1088/1361-6528/ab9390.

284 A. X. Cartagena-Rivera, W. H. Wang, R. L. Geahlen and A. Raman, Fast, Multi-Frequency, and Quantitative Nanomechanical Mapping of Live Cells Using the Atomic Force Microscope, Sci. Rep., 2015, 5, 1-11.

285 M. O. Krisenko, A. Cartagena, A. Raman and R. L. Geahlen, Nanomechanical property maps of breast cancer cells as determined by multiharmonic atomic force microscopy reveal Syk-dependent changes in microtubule stability mediated by MAP1B, Biochemistry, 2015, 54, 60-68.

286 A. Cartagena, M. Hernando-Pérez, J. L. Carrascosa, P. J. de Pablo and A. Raman, Mapping in vitro local material properties of intact and disrupted virions at high resolution using multi-harmonic atomic force microscopy, Nanoscale, 2013, 5, 4729-4736.

287 D. A. Chernoff, High resolution chemical mapping using tapping mode AFM with phase contrast, Proceedings of Microscopy and Microanalysis, Jones and Begell, New York, 1995, pp. 888-889.

288 G. Bar, Y. Thomann and M. H. Whangbo, Characterization of the morphologies and nanostructures of blends of polystyrene-block-poly(ethane-co-but-1-ene)-blocl-

polystyrene with isotactic and atactic polypropylenes by tapping-mode AFM, Langmuir, 1998, 14, 1219.

289 P. Leclère, A. Calderone, D. Marsitzky, V. Francke, Y. Geerts, K. Müllen, J. L. Brédas and R. Lazzaroni, Highly Regular Organization of Conjugated Polymer Chains via Block Copolymer Self-Assembly, Adv. Mater., 2000, 12, 1042-1046.

290 G. Reiter, G. Castelein, J. U. Sommer, A. Röttele and T. Thurn-Albrecht, Direct visualization of random crystallization and melting in arrays of nanometer-size polymer crystals, Phys. Rev. Lett., 2001, 87, 226101.

291 G. Krausch and R. Magerle, Nanostructured thin films via self-assembly of block copolymers, Adv. Mater., 2002, 14, 1579.

292 D. Sundrani, S. B. Darling and S. J. Sibener, Guiding to perfection: Macroscopi alignment of nanoscale domains, Nano Lett., 2004, 4, 273-276.

293 A. Knoll, R. Magerle and G. Krausch, Phase behavior in thin films of cylinder-forming block copolymers: experiments, J. Chem. Phys., 2004, 120, 1105-1116.

294 A. San Paulo and R. Garcia, Tip-Surface Forces, Amplitude, and Energy Dissipation in Amplitude-Modulation (Tapping Mode) Force Microscopy, Phys. Rev. B: Condens. Matter Mater. Phys., 2001, 64, 193411.

295 J. Tamayo and R. Garcia, Effects of elastic and inelastic interactions on phase contrast images in tapping-mode scanning force microscopy, Appl. Phys. Lett., 1997, 71, 2394.

296 J. Tamayo and R. Garcia, Relationship between phase shift and energy dissipation in tapping-mode scanning force microscopy, Appl. Phys. Lett., 1998, 73, 2926.

297 J. P. Cleveland, B. Anczykowski, A. E. Schmid and V. B. Elings, Energy dissipation in tapping-mode atomic force microscopy, Appl. Phys. Lett., 1998, 72, 2613.

298 S. N. Magonov, V. Elings and M. H. Whangbo, Phase imaging and stiffness in tapping-mode atomic force microscopy, Surf. Sci., 1997, 375, L385.

299 R. Brandsch, G. Bar and M. H. Whangbo, On the factors affecting the contrast of height and phase images in tapping mode atomic force microscopy, Langmuir, 1997, 13, 6349.

300 R. Garcia, J. Tamayo, M. Calleja and F. Garcia, Phase contrast in tapping-mode scanning force microscopy, Appl. Phys. A: Mater. Sci. Process., 1998, 66, S312. 
301 O. P. Behrend, L. Odoni, J. L. Loubet and N. A. Burnham, Phase imaging: deep or superficial?, Appl. Phys. Lett., 1999, 75, 2551.

302 M. Stark, C. Moller, D. J. Muller and R. Guckenberger, From images to interactions: High-resolution phase imaging in tapping-mode atomic force microscopy, Biophys. J., 2001, 80, 3009.

303 P. J. James, M. Atognozzi, J. Tamayo, T. J. McMaster, J. M. Newton and M. J. Miles, Interpretation of contrast in tapping mode AFM and shear force microscopy, A study of Nafion, Langmuir, 2001, 17, 349.

304 K. Schröter, A. Petzold, T. Henze and T. Thurn-Albrecht, Quantitative analysis of scanning force microscopy data using harmonic models, Macromolecules, 2009, 42, 1114.

305 Y. Zhao, Q. Cheng, M. Qian and J. H. Cantrell, Phase image contrast mechanism in intermittent contact atomic force microscopy, J. Appl. Phys., 2010, 108, 094311.

306 J. Tamayo, Energy dissipation in tapping-mode scanning force microscopy with low quality factors, Appl. Phys. Lett., 1999, 75, 3569-3571.

307 J. Melcher, X. Xu, A. Raman, C. Carrasco-Pulido, J. GomezHerrero, P. J. de Pablo and J. L. Carrascosa, Origins of phase contrast in the atomic force microscope in liquids, Proc. Natl. Acad. Sci. U. S. A., 2009, 106, 13655.

308 A. F. Payam, J. R. Ramos and R. Garcia, Molecular and Nanoscale Compositional Contrast of Soft Matter in Liquid: Interplay between Elastic and Dissipative Interactions, ACS Nano, 2012, 6, 4663-4670.

309 B. Anczykowski, B. Gotsman, H. Fuchs, J. P. Cleveland and V. B. Elings, How to measure energy dissipation in dynamic mode atomic force microscopy, Appl. Surf. Sci., 1999, 140, 376.

310 N. F. Martinez and R. Garcia, Measuring phase shifts and energy dissipation with amplitude modulation atomic force microscopy, Nanotechnology, 2006, 17, S167.

311 E. Sahagun, P. Garcia-Mochales, G. M. Sacha and J. J. Saenz, Energy dissipation due to capillary interactions: Hydrophobicity maps in force microscopy, Phys. Rev. Lett., 2007, 98, 176106.

312 C. J. Gomez and R. Garcia, Determination and simulation of nanoscale energy dissipation processes in amplitude modulation AFM, Ultramicroscopy, 2010, 110, 626-633.

313 S. Santos, V. Barcons, A. Verdaguer, J. Font, N. H. Thomson and M. Chiesa, How localized are energy dissipation processes in nanoscale interactions?, Nanotechnology, 2011, 22, 345401.

314 D. Wang, X. B. Liang, Y. H. Liu, T. Nishi and K. Nakajima, Characterization of Surface Viscoelasticity and Energy Dissipation in a Polymer Film by Atomic Force Microscopy, Macromolecules, 2011, 44, 8693-8697.

315 F. Lavini, F. Cellini, M. Rejhon, J. Kunc, C. Berger, W. de Heer and E. Riedo, Atomic Force Microscopy Phase Imaging of Epitaxial Graphene films, J. Phys.: Mater., 2020, 3, 024005.

316 R. Proksch and D. G. Yablon, Loss tangent imaging: Theory and simulations of repulsive-mode tapping atomic force microscopy, Appl. Phys. Lett., 2012, 100, 073106.
317 R. Proksch, M. Kocun, D. C. Hurley, M. Viani, A. Labuda, W. Meinhold and J. Bemis, Practical Loss Tangent Imaging with Amplitude-Modulated Atomic Force Microscopy, J. Appl. Phys., 2016, 119, 134901.

318 E. C. Spitzner, C. Riesch and R. Magerle, Subsurface Imaging of Soft Polymeric Materials with Nanoscale Resolution, ACS Nano, 2011, 5, 315-320.

319 M. Dehnert, E. C. Spitzner, F. Beckert and R. Magerle, Subsurface Imaging of Functionalized and Polymer-Grafted Graphene Oxide, Macromolecules, 2016, 49, 7415-7425.

320 E. C. Spitzner, S. Röper, M. Zerson, A. Bernstein and R. Magerle, Nanoscale Swelling Heterogeneities in Type I Collagen Fibrils, ACS Nano, 2015, 9, 5683-5694.

321 M. R. Uhlig and R. Magerle, Unraveling Capillary Interaction and Viscoelastic Response in Atomic Force Microscopy of Hydrated Collagen Fibrils, Nanoscale, 2017, 9, 1244-1256.

322 H. K. Nguyen, X. Liang, M. Ito and K. Nakajima, Direct Mapping of Nanoscale Viscoelastic Dynamics at Nanofiller/ Polymer Interfaces, Macromolecules, 2018, 51, 6085-6091.

323 H. K. Nguyen, M. Ito, S. Fujinami and K. Nakajima, Viscolasticity of inhomogenous polymers characterized by loss tangent measurements using AFM, Macromolecules, 2014, 47, 7971-7977.

324 J. G. Raybin, J. G. Murphy, M. Dolejsi and S. J. Sibener, Direct Imaging of Interfacial Fluctuations in Confined Block Copolymer with in Situ Slow-Scan-Disabled Atomic Force Microscopy, ACS Nano, 2019, 13, 11741-11752.

325 G. L. Alharbe, R. A. Register and J. K. Hobbs, Orientation Control and Crystallization in a Soft Confined Phase Separated block copolymer, Macromolecules, 2017, 50, 987-996.

326 J. Raybin, J. Ren, X. Chen, R. Gronheid, P. F. Nealey and S. J. Sibener, Real-time atomic force microscopy imaging of block copolymer directed self assembly, Nano Lett., 2017, 17, 7717-7723.

327 Y. Kikuchi, N. Obana, M. Toyofuku, N. Kodera, T. Soma, T. Ando, Y. Fukumori and T. Nomura, Diversity of physical properties of bacterial extracellular membrane vesicles revealed through atomic force microscopy phase imaging, Nanoscale, 2020, 12, 7950-7959.

328 D. J. Muller, J. B. Heymann, F. Oesterhelt, C. Moller and H. Gaub, G. and A. Engel, Atomic force microscopy of native purple membrane, Biochim. Biophys. Acta, 2000, 1460, 27-38.

329 J. L. Hutter and J. Bechhoefer, Calibration of atomic-force microscope tips, Rev. Sci. Instrum., 1993, 64, 1868.

330 H. J. Butt and M. Jaschke, Calculation of thermal noise in atomic force microscopy, Nanotechnology, 1995, 6, 1-7.

331 S. M. Cook, T. E. Schäffer, K. M. Chynoweth, M. Wigton, R. W. Simmonds and K. M. Lang, Practical implementation of dynamic methods for measuring atomic force microscope cantilever spring constants, Nanotechnology, 2006, 17, 2135-2145.

332 J. E. Sader, R. Borgani, C. T. Gibson, D. B. Haviland, M. J. Higgins, J. I. Kilpatrick, J. Lu, P. Mulvaney, C. J. Shearer, 
A. D. Slattery, P.-A. Thorén, J. Tran, H. Zhang, H. Zhang and T. Zheng, A virtual instrument to standardise the calibration of atomic force microscope cantilevers, Rev. Sci. Instrum., 2016, 87, 093711.

333 J. R. Lozano, D. Kiracofe, J. Melcher, R. Garcia and A. Raman, Calibration of higher eigenmode spring constants of atomic force microscope cantilevers, Nanotechnology, 2010, 21, 465502.

334 A. Labuda, M. Kocun, M. Lysy, T. Walsh, J. Meinhold, T. Proksch, W. Meinhold, C. Anderson and R. Proksch, Calibration of Higher Eigenmodes of Cantilevers, Rev. Sci. Instrum., 2016, 87, 073705.

335 J. Liu, J. K. Notbohm, R. W. Carpick and K. T. Turner, Method for characterizing nanoscale wear of atomic force microscope tips, ACS Nano, 2010, 4, 3763-3772.

336 S. Santos, L. Guang, T. Souier, K. Gadelrab, M. Chiesa and N. H. A. Thomson, Method to Provide Rapid in Situ Determination of Tip Radius in Dynamic Atomic Force Microscopy, Rev. Sci. Instrum., 2012, 83, 043707.

337 M. Li, N. Xi, Y. Wong and L. Liu, Advances in atomic force microscopy for single-cell analysis, Nano Res., 2019, 12, 703-714.

338 D. R. Chery, B. Han, Q. Li, Y. Zhou, S. J. Heo, B. Kwok, P. Chandrasekaran, C. Wang, L. Qin, L. D. Kong, M. Enomoto-Iwamoto, R. L. Mauck and L. Han, Early Changes in Cartilage Pericellular Matrix Micromechanobiology Portend the Onset of Post-Traumatic Osteoarthritis, Acta Biomater., 2020, 111, 267-278.

339 I. Jorba, J. J. Uriarte, N. Campillo, R. Farre and D. Navajas, Probing micromechanical properties of the extracellular matrix of soft tissues by AFM, J. Cell Phys., 2016, 232, 19-26.

340 B. Han, et al., Decorin regulates teh aggrecan network integrity and biomechanical functions of cartilage extracellular matrix, ACS Nano, 2019, 13, 11320-11333.

341 R. P. Chartoff, J. D. Menczel and S. H. Dillman, Dynamic mechanical analysis (DMA), ed. J. D. Menczel and R. B. Prime, Thermal Analysis of Polymers, John Wiley \& Sons, Inc, Hoboken, New Jersey, 2009, pp. 387-495.

342 J. D. Ferry, Viscoelastic properteis of polymers, John Wiley \& Sons, 3rd edn, 1980.

343 J. B. Pethica, R. Hutchings and W. C. Oliver, Hardness measurement at penetration depths as small as $20 \mathrm{~nm}$, Philos. Mag. A, 1983, 48, 593-606.

344 P. Schön, K. Molnár, P. Markus, B. Pukánszky and G. J. Vancso, Quantitative mapping of elastic moduli at the nanoscale in phase separated polyurethanes by AFM, Eur. Polym. J., 2011, 47, 692-698.

345 M. E. Dokukin and I. Sokolov, Quantitative mapping of the elastic modulus of soft materials with HarmoniX and PeakForce QNM AFM modes, Langmuir, 2012, 28, 16060-16071.

346 T. J. Young, T. J. M. A. Monclus, T. L. Burnett, W. R. Broughton, S. L. Ogin and P. A. Smith., The use of the PeakForce $^{\mathrm{TM}}$ quantitative nanomechanical mapping AFMbased method for high-resolution Young's modulus measurement of polymers, Meas. Sci. Technol., 2011, 22, 125703.
347 B. Pittenger, S. Osechinskiy, D. Yablon and T. Mueller, Nanoscale DMA with the atomic force microscope: A new method for measuring viscoelastic properties of nanostructured polymer materials, JOM, 2019, 71, 3390-3398.

348 M. E. Dokukin and I. Sokolov, On the Measurements of Rigidity Modulus of Soft Materials in Nanoindentation Experiments at Small Depth, Macromolecules, 2012, 45, 4277-4288.

349 M. Griepentrog, G. Kramer and B. Cappella, Comparison of nanoindentation and AFM methods for the determination of mechanical properties of polymers, Polym. Test., 2013, 32, 455-460.

$350 \mathrm{H}$. Askes and E. C. Aifantis, Gradient elasticity in statics and dynamics: an overview of formulations, length scale identification procedures, finite element implementations and new results, Int. J. Solids Struct., 2011, 48, 1962-1990.

351 M. Li, L. Liu, X. Xiao, N. Xi and Y. Wang, Viscoelastic properties measurement of human lymphocites by AFM based on magnetic beads cell isolation, IEEE Trans. NanoBiosci., 2016, 15, 1236-1241.

352 H. T. Nia, I. S. Bozchalooi, Y. Li, L. Han, H. H. Hung, E. Frank, K. Youcemi-Toumi, C. Ortiz and A. Grodzinsky, High-Bandwidth AFM-Based Rheology Reveals that Cartilage is Most Sensitive to High Loading Rates at Early Stages of Impairment, Biophys. J., 2013, 104, 1529-1537.

353 B. Han, H. T. Nia, C. Wang, P. Chandrasekaran, Q. Li, D. R. Chery, H. Li, A. J. Grodzinsky and L. Han, AFMNanomechanical Test: An Interdisciplinary Tool That Links the Understanding of Cartilage and Meniscus Biomechanics, Osteoarthritis Degeneration, and Tissue Engineering, ACS Biomater. Sci. Eng., 2017, 3, 2033-2049.

354 V. G. Gisbert, S. Benaglia, M. R. Uhlig, R. Proksch and R. Garcia, private communication.

355 R. E. Wilusz, L. E. DeFrate and F. Guilak, Immunofluorescence-guided atomic force microscopy to measure the micromechanical properties of the perinuclear matrix of porcine articular cartilage, J. R. Soc., Interface, 2012, 9, 2997-3007.

356 S. W. Hell, Microscopy and its focal switch, Nat. Methods, 2009, 6, 24-32.

357 S. V. Kalinin, E. Strelcov, A. Belianinov, S. Somnath, R. K. Vasudevan, E. J. Lingerfelt, R. K. Archibald, C. Chen, R. Proksch, N. Laanait and S. Jesse, Big, Deep, and Smart Data in Scanning Probe Microscopy, ACS Nano, 2016, 10, 9068-9086.

358 S. Santos, C. Y. Lai, T. Olukan and M. Chiesa, Multifrequency AFM: from origins to convergence, Nanoscale, 2017, 9, 5038-5043.

359 I. Sokolov, M. E. Dokukin, V. Kalaparthi, M. Miljkovica, A. Wanga, J. D. Seigned, P. Grivase and E. Demidenkof, Noninvasive diagnostic imaging using machine-learning analysis of nanoresolution images of cell surfaces: Detection of bladder cancer, Proc. Natl. Acad. Sci. U. S. A., 2018, 115, 12920-12925.

360 C. Y. Lai, S. Santos and M. Chiesa, Machine learning assisted quantification of graphitic surfaces exposure to defined environments, Appl. Phys. Lett., 2019, 114, 241601. 